\title{
Explicit calculation of Frobenius isomorphisms and Poincaré duality in the theory of arithmetic $\mathscr{D}$-modules
}

\author{
TOMOYuki ABE (*)
}

ABSTRACT - The aim of this paper is to compute the Frobenius structures of some cohomological operators of arithmetic $\mathscr{D}$-modules. To do this, we calculate explicitly an isomorphism between canonical sheaves defined abstractly. Using this calculation, we establish the relative Poincaré duality in the style of SGA4. As another application, we compare the push-forward as arithmetic $\mathscr{D}$-modules and the rigid cohomologies taking Frobenius into account. These theorems will be used to prove " $p$-adic Weil II" and a product formula for $p$-adic epsilon factors.

Mathematics Subject Classification (2010). 14F30.

KEYworDs. p-adic cohomology, arithmetic $\mathscr{D}$-module, rigid cohomology.

\section{Introduction}

In this paper, we prove several results concerning Frobenius structures in the theory of arithmetic $\mathscr{D}$-modules. There are mainly three goals in this paper.

(G1) Compute and describe Frobenius structures of some cohomological operators, appearing in [Be2], concretely in terms of differential operators.

(G2) Establish a relative Poincaré duality in the style of SGA4 in the theory of arithmetic $\mathscr{D}$-modules.

(G3) Compare the push-forwards in the theory of $\mathscr{D}$-modules and the rigid cohomologies with Frobenius structure.

(*) Indirizzo dell'A.: Institute for the Physics and Mathematics of the Universe (WPI), The University of Tokyo, 5-1-5 Kashiwanoha, Kashiwa, Chiba, 277-8583, Japan.

E-mail: tomoyuki.abe@ipmu.jp 
First, (G1) is the starting point of the other two goals. We describe some isomorphisms appearing in [Be2] explicitly by taking local coordinates. Apart from (G2) and (G3), this calculation is used in [AM] to compute the geometric Fourier transform defined by C. Noot-Huyghe explicitly. With this description, we are able to re-prove Gross-Koblitz formula [GK] using arithmetic $\mathscr{D}$-modules. This calculation will be discussed in other places. We expect that these ideas can be generalized to a calculation of $p$-adic $\varepsilon$-factors.

For (G2), a duality theory was established by A. Virrion in [Vi2] to some extent. However, we need two more ingredients to call it the Poincaré duality: 1) comparison of the extraordinary pull-back and the normal pullback for a smooth morphism, and 2) taking Frobenius structures into account. Using our result 1) on the comparison of two types of pull-backs, we are also able to compare duality functors of the theory of rigid cohomology and that of arithmetic $\mathscr{D}$-modules, which completes a work in [Ca5]. For 2), even without Frobenius structures, her duality is very powerful tool, but in practical uses of arithmetic $\mathscr{D}$-module theory, Frobenius structure is another important ingredient that contain arithmetic information. For example, $L$-functions for holonomic $\mathscr{D}^{\dagger}$-modules cannot be defined without Frobenius structures, and thus to show the functional equation for $L$ functions, it is necessary to consider Frobenius structures in the duality.

(G3) is another application of (G1). If we do not consider Frobenius structures, this is a well-known result of Berthelot [BeI, 4.3.6.3]. This type of comparison theorem is necessary when we want to exploit results of the theory of $\mathscr{D}$-modules in the theory of rigid cohomologies and vice versa. For example, in [Ca2, 3.3], the author discussed the relations of $L$-functions defined using the theory of rigid cohomologies and that of arithmetic $\mathscr{D}$ modules. This result can be reinforced and re-stated much clearer using our result (cf. Remark 3.12). For the situation of this result, see also Remark 3.15 .

Now, let us go into more details of the results. Let $R$ be a complete discrete valuation ring of mixed characteristic $(0, p)$, and we denote by $k$ its residue field which is assumed to be perfect, $K$ its field of fractions. Let $\mathscr{C}$ be a smooth formal scheme over $\operatorname{Spf}(R)$, and $X_{0}$ be the reduction of $\mathscr{C}$ over $k$. Let $s$ be a positive integer, and we put $X_{0}^{\prime}:=X_{0} \otimes_{k, F_{k}^{s}} k$ where $F_{k}^{s}: \operatorname{Spec}(k) \rightarrow \operatorname{Spec}(k)$ denotes the $s$-th absolute Frobenius isomorphism. In this introduction, we also assume that there exist liftings $\sigma: \operatorname{Spf}(R) \stackrel{\sim}{\rightarrow}$ $\operatorname{Spf}(R)$ of $F_{k}^{s}$ and $F: \mathscr{C} \rightarrow \mathscr{C}^{\prime}:=\mathscr{C} \otimes_{R, \sigma} R$ of the relative Frobenius morphism $F_{X_{0} / k}^{s}: X_{0} \rightarrow X_{0}^{\prime}$ for simplicity. A coherent $F$ - $\mathscr{D}_{\mathscr{X}, \mathrm{Q}}^{\dagger}$-module is a 
couple of a coherent $\mathscr{D}_{\mathscr{C}, Q}^{\dagger}$-module $\mathscr{L} b$ and an isomorphism $F^{*} \mathscr{L}^{\sigma} \stackrel{\sim}{\rightarrow} \mathscr{L}$ where $\mathscr{L}^{\sigma}$ denotes the $\mathscr{D}_{\mathscr{C}^{\prime}, \mathrm{Q}}^{\dagger}$-module induced by $\mathscr{C}$ by the base change $\sigma$. Frobenius structures are known to be stable under reasonable cohomological operations of arithmetic $\mathscr{D}$-modules such as push-forwards, extraordinary pull-backs, tensor products, etc (cf. [Be2]).

When we try to calculate Frobenius structures of some cohomological operations (e.g. push-forward functor), an obstacle lies in the isomorphism $\omega_{\mathscr{K}} \stackrel{\sim}{\rightarrow} F^{b} \omega_{\mathscr{O}^{\prime}}$ of $[\mathrm{Be} 2,2.4 .2]$. The construction of this isomorphism is formal using general facts of [Ha]. However, we need to trace many isomorphisms of [Ha] to compute it explicitly, which is monotonous but messy. The advantage of this computation is that it makes us possible to calculate Frobenius structures in "brutal" but very direct ways, at least locally. As an example of the explicit computation, we calculate the Frobenius structure of push-forwards (cf. paragraph 2.5). We can also prove a proper base change type lemma (cf. Lemma 2.6). With an aid of a result of Caro, we get the proper base change theorem in paragraph 5.7. Another application will be to prove the following theorem.

THEOREM 3.10. Let $f: \mathscr{C} \rightarrow \mathscr{Y}$ be a smooth morphism of relative dimension $d$ between smooth formal schemes. For a coherent $F-\mathscr{D}_{\mathscr{Y}, Q^{-}}^{\dagger}$ module llb,

$$
f^{!}\left(\mathbb{D}_{\mathscr{Y}}(\mathscr{C O})\right) \cong \mathbb{D}_{\mathscr{C}}\left(f^{!} \mathscr{C O}\right)(d)[2 d]
$$

where (d) denotes the d-th Tate twist (cf. paragraph 2.7).

The construction of the isomorphism without Frobenius structures requires only standard methods of the theory of arithmetic $\mathscr{D}$-modules, but to see the compatibility with Frobenius structures, we need the explicit calculation of the isomorphism of canonical sheaves. Using a result we get on the way we prove this theorem, we compare the rigid cohomologies and the push-forwards in the arithmetic $\mathscr{D}$-module theory, which is (G3). This theorem can be seen as a part of Poincaré duality. See the last section for an account of this interpretation. Moreover this theorem leads us to complete a work of Caro in [Ca3] (cf. Corollary 3.12) comparing the duality functors in the theory of rigid cohomology and that of arithmetic $\mathscr{D}$-modules.

In this paper, we also include some small but useful results concerning Frobenius pull-backs. Namely, we prove: 1. commutation of the dual functor and the tensor product in some cases, 2. the Künneth formula, 3. compatibility of the relative duality homomorphism with Frobenius. The result 1 uses (甲) in the proof, but results 2 and 3 are independent of the 
explicit computations. The results 1 and 2 are included in this paper with the intention of use in $[\mathrm{AM}]$. The result 3 is aimed to establish the Poincaré duality as we have already mentioned.

Finally, let us point out some notable applications of our results. Currently, we have the following two important applications:

- establishing the "yoga of weights" in $p$-adic cohomologies, especially an analog of "Weil II" in the theory of arithmetic $\mathscr{D}$-modules. This will be treated in a paper of the author jointly with D. Caro (see $[\mathrm{AC}])$.

- a product formula for $p$-adic epsilon factors. See [AM] for more details.

In the proofs of those two results, (G2) and (G3) are used extensively.

Let us see the structure of this paper. In $\S 1$, we describe isomorphisms which are key isomorphisms to construct the commutativity. In $\S 2$, we calculate the Frobenius structure of push-forwards explicitly. We should mention that this calculation is a key to calculate the Frobenius structure of Fourier transforms explicitly, which is carried out in [AM]. With these two sections, (G1) is attained. As an application, we show a proper base change type lemma also in this section. In §3, we show that the dual functor and the extraordinary pull-back functor commute up to some degree shift and Tate twist in the smooth case. Using a lemma we prove to show this commutativity, we will compare the rigid cohomology and the push-forward of arithmetic $\mathscr{D}$ modules, and we get (G3). In §4, we will show some complementary results, which are used in [AM]. The idea of the proof of the Künneth formula is due to P. Berthelot. In this section, we also prove that the relative duality isomorphism of Virrion is compatible with Frobenius. Together with §3, (G2) is completed. In §5, we interpret the results in terms of the philosophy of "six functors" by Grothendieck, which clarifies the meaning of the results in this paper. We refer to the recent paper [Ab2] for more complete treatment.

\section{Notation}

0.1. In this paper we fix a complete discrete valuation ring $R$ with mixed characteristic $(0, p)$. We denote the residue field by $k$, the field of fractions by $K$. For a non-negative integer $i$, we put $R_{i}$ to be $R / \pi^{i+1} R$ where $\pi$ is a uniformizer. We denote by $e$ the absolute ramification index of $K$.

In general, we use Roman fonts (e.g. X) for schemes and script fonts $($ e.g. $\mathscr{C})$ for formal schemes. For a formal scheme $\mathscr{C}$ over $\operatorname{Spf}(R)$, we usually denote by $X_{i}$ the reduction $\mathscr{C} \otimes_{R} R_{i}$ over $\operatorname{Spec}\left(R_{i}\right)$. 
0.2. For a scheme $X$ over $\operatorname{Spec}(k)$, we denote by $F_{X}: X \rightarrow X$ the absolute Frobenius homomorphism: it sends a section $f$ of $\mathcal{O}_{X}$ to $f^{p}$. We fix a positive integer $s$, and put $q:=p^{s}$. We put $X^{(s)}:=X \otimes_{k, F_{k}^{s *}} k$, and call it the relative $s$-th Frobenius of $X$.

0.3. Let $\mathscr{D}$ be a sheaf of rings on a topological space $X$. When we simply say $\mathscr{D}$-module, it means left $\mathscr{D}$-module. We denote by $D_{\text {coh }}^{*}(\mathscr{D})$ $(* \in\{+,-, b\})$ the full subcategory of $D^{*}(\mathscr{D})$ such that the objects consist of complexes whose cohomology sheaves are coherent. We denote by $D_{\text {perf }}(\mathscr{D})$ the full subcategory whose objects consist of perfect complexes (i.e. complexes locally quasi-isomorphic to bounded complexes of locally projective $\mathscr{D}$-modules). We denote by $D_{\mathrm{ftd}}(\mathscr{D})$ the full subcategory consisting of finite Tor-dimensional complexes (i.e. complexes possessing bounded flat resolutions). We put $D_{\text {perf }}^{b}(\mathscr{D}):=$ $D_{\text {perf }}(\mathscr{D}) \cap D_{\mathrm{ftd}}(\mathscr{D})$. When $X$ is quasi-compact and $\mathscr{D}$ is coherent, $D_{\text {perf }}^{b}(\mathscr{D})$ coincides with $D_{\text {perf }}(\mathscr{D}) \cap D_{\text {coh }}^{b}(\mathscr{D})$. See SGA6 Exp. I for details. When we denote by $D(\mathscr{D})^{\mathrm{g}}\left(\right.$ resp. $\left.D(\mathscr{D})^{\mathrm{d}}\right)$ we consider complexes of left (resp. right) $\mathscr{D}$-modules ( $\mathrm{g}$ and $\mathrm{d}$ stand for French words "gauche" and "droit"). When we put $Q$ as an index, this means tensor with Q.

0.4. In this paper, we freely use the language of arithmetic $\mathscr{D}$-modules. For details see [Be1], [Be2], [BeI]. In particular, we use the rings $\mathscr{D}_{X}^{(m)}$, $\hat{\mathscr{D}}_{\mathscr{X}}^{(m)}, \mathscr{D}_{\mathscr{X}}^{\dagger}$ for a smooth scheme $X$ and a smooth formal scheme $\mathscr{X}$. We use the category $L D_{\mathrm{Q}, \mathrm{qc}}^{b}\left(\widehat{\mathscr{D}}_{\mathscr{\mathscr { C }}}^{(\bullet)}\right)$ whose definition is written in [BeI, 4.2]. Let $Z$ be a divisor of the special fiber of $\mathscr{K}$. We denote $\widehat{\mathcal{B}}_{\mathscr{X}}^{(m)}(Z) \widehat{\otimes}$ $\widehat{\mathscr{D}}_{\mathscr{K}}^{(m)}:=\lim _{i} \mathcal{B}_{X_{i}}(Z) \otimes \mathscr{D}_{X_{i}}^{(m)}$ by $\widehat{\mathscr{D}}_{\mathscr{C}}^{(m)}(Z)$, and $\widehat{\mathscr{D}}_{\mathscr{C}}^{(m)}(Z) \otimes \mathrm{Q}$ by $\widehat{\mathscr{D}}_{\mathscr{X}, \mathrm{Q}}^{(m)}(Z)$. By the same construction as $\underset{L D_{\mathrm{Q}, \mathrm{qc}}^{b}}{b}\left(\widehat{\mathscr{D}}_{\mathscr{X}}^{(\bullet)}\right)$, we can consider the category

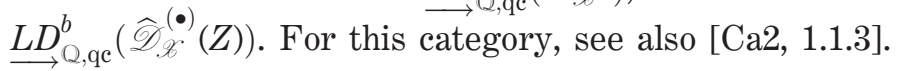

0.5. Let $\mathscr{C}$ be a smooth formal scheme, and $Z$ be a divisor of its special fiber. Let $\mathscr{C}:=\mathscr{C} \backslash Z, X$ and $U$ be the special fibers of $\mathscr{C}$ and $\mathscr{C}$ respectively. Let $\mathscr{C}$ be a coherent $\left(F_{-}\right) \mathscr{D}_{\mathscr{X}, Q}^{\dagger}\left({ }^{\dagger} Z\right)$-module such that it is coherent as an $\left.\mathcal{O}_{\mathscr{K}, \mathrm{Q}}{ }^{\dagger} Z\right)$-module. Let $\mathcal{C}$ be the full subcategory of the category of coherent $(F-) \mathscr{D}_{\mathscr{X}, \mathrm{Q}}^{\dagger}\left({ }^{\dagger} Z\right)$-modules consisting of such $\mathscr{l}$. Then we know that the specialization functor induces an equivalence between $\mathcal{C}$ and the category $(F-) \operatorname{Isoc}^{\dagger}(U, X / K)$ by $[\mathrm{Be} 1,4.4 .12]$ and $[\mathrm{Be} 2,4.6 .3,4.6 .7]$. We say that $\mathscr{C l}$ is a convergent $(F$-)isocrystal on $\mathscr{C b}$ overconvergent along $Z$ by abuse of language. 


\section{Explicit calculation of isomorphisms of canonical sheaves}

In this section, we will explicitly calculate the isomorphisms of [Be2, 2.4.3, 2.4.4] (cf. Theorem 1.7), from which some commutation results of Berthelot [Be2] are derived. The existence of these isomorphisms are direct consequences of fundamental properties of the functors of Hartshorne [Ha], and for the explicit calculations, we need to go back to the proofs of these fundamental properties, and trace these isomorphisms step by step. We follow the notation of [Ha, III].

1.1. First we review the notation and functors of Hartshorne [Ha, III] in short. Let $f: X \rightarrow Y$ be a morphism of schemes. When $f$ is smooth, we denote by $\omega_{X / Y}$ the canonical sheaf $\Lambda^{d} \Omega_{X / Y}$ where $d$ denotes the relative dimension of $f$. When $f$ is regular closed immersion, let $\mathcal{J}$ be the sheaf of ideals of $\mathcal{O}_{Y}$ defining $X$. Then we put $\omega_{X / Y}:=\left(\bigwedge^{d} \mathcal{J} / \mathcal{J}^{2}\right)^{\vee}$ where $d$ is the codimension of $X$ in $Y$, and ${ }^{\vee}$ is the dual as an $\mathcal{O}_{X}$-module. In both cases, $\omega_{X / Y}$ is a locally free $\mathcal{O}_{X}$-module of rank 1.

Suppose $f$ is smooth. We define a functor $f^{\sharp}: D\left(\mathcal{O}_{Y}\right) \rightarrow D\left(\mathcal{O}_{X}\right)$ as follows. See [Ha, III § 2] for more details. For $C \in D\left(\mathcal{O}_{Y}\right)$, we put $f^{\sharp}(C):=f^{*}(C) \otimes_{\mathcal{O}_{X}} \omega_{X / Y}[d]$ where $d$ is the relative dimension of $f$. We see that this functor takes $D_{\mathrm{qc}}^{b}\left(\mathcal{O}_{Y}\right)$ into $D_{\mathrm{qc}}^{b}\left(\mathcal{O}_{X}\right)$ (here $D_{\mathrm{qc}}^{*}$ denotes the full subcategory of the derived category consisting of objects whose cohomologies are quasi-coherent sheaves).

In turn, suppose $f$ is a finite morphism. We denote by $\bar{f}$ the morphism of ringed spaces $\left(X, \mathcal{O}_{X}\right) \rightarrow\left(Y, f_{*} \mathcal{O}_{X}\right)$. Then we define a functor $f^{b}: D^{+}\left(\mathcal{O}_{Y}\right) \rightarrow$ $D^{+}\left(\mathcal{O}_{X}\right)$ as follows. See [Ha, III $\left.\S 6\right]$ for more details. For $C \in D^{+}\left(\mathcal{O}_{Y}\right)$, we put $f^{\prime}(C):=\bar{f}^{*} R \mathcal{H}^{\prime} m_{\mathcal{O}_{Y}}\left(f_{*} \mathcal{O}_{X}, C\right)$. We know that this functor takes $D_{\mathrm{qc}}^{+}\left(\mathcal{O}_{Y}\right)$ into $D_{\mathrm{qc}}^{+}\left(\mathcal{O}_{X}\right)$, and if $f$ has finite Tor-dimension (cf. [Ha, II $\S 4$ ], e.g. flat morphism), then it takes bounded complexes into bounded complexes.

1.2. Now, consider the following diagram of schemes

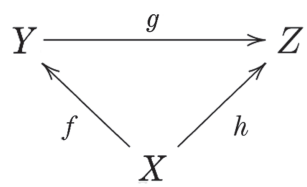

where $f$ and $h$ are regular closed immersions of codimension $d>0$, and $g$ is a finite flat morphism. Since $g$ is finite flat, we get that

$$
g^{b}\left(\mathcal{O}_{Z}\right) \cong \bar{g}^{*} \mathcal{H o m}_{\mathcal{O}_{Z}}\left(g_{*} \mathcal{O}_{Y}, \mathcal{O}_{Z}\right)
$$


There exists the natural equivalence $f^{b} g^{b} \cong h^{b}$ by [Ha, III Proposition 6.2]. By taking the $d$-th cohomology, we get an isomorphism

$$
\imath: \bar{f}^{*} \mathcal{E} x t_{\mathcal{O}_{Y}}^{d}\left(f_{*} \mathcal{O}_{X}, \bar{g}^{*} \mathcal{H o m}_{\mathcal{O}_{Z}}\left(g_{*} \mathcal{O}_{Y}, \mathcal{O}_{Z}\right)\right) \stackrel{\sim}{\rightarrow} \bar{h}^{*} \mathcal{E} x t_{\mathcal{O}_{Z}}^{d}\left(h_{*} \mathcal{O}_{X}, \mathcal{O}_{Z}\right) .
$$

Now, suppose $Z$ is an affine scheme. Then the other two schemes are also affine schemes. We denote the global sections of $X$ (resp. $Y, Z$ ) by $R_{X}$ (resp. $R_{Y}, R_{Z}$ ). By [Ha, III, Proposition 6.1], the source and target of $\iota$ are quasi-coherent $\mathcal{O}_{X}$-modules. Thus, $l$ is associated to the following isomorphism of $R_{X}$-modules

$$
\operatorname{Ext}_{R_{Y}}^{d}\left(R_{X}, \operatorname{Hom}_{R_{Z}}\left(R_{Y}, R_{Z}\right)\right) \stackrel{\sim}{\rightarrow} \operatorname{Ext}_{R_{Z}}^{d}\left(R_{X}, R_{Z}\right),
$$

and we also denote this isomorphism by $\imath$. We calculate this isomorphism in terms of the fundamental local isomorphism [Ha, III, Proposition 7.2].

Suppose moreover that there exists a system of local parameters defining $X$ in $Y$ (resp. in $Z$ ) denoted by $\left\{y_{i}\right\}_{i<i<d}$ (resp. $\left\{z_{i}\right\}_{1<i<d}$ ). Let $I:=\operatorname{Ker}\left(R_{Y} \rightarrow R_{X}\right)$. The sheaf $\omega_{X / Y}$ is the quasi-coherent sheaf associated to $\operatorname{Hom}_{R_{X}}\left(\bigwedge^{d} I / I^{2}, R_{X}\right)$. Since $y_{1} \wedge \cdots \wedge y_{d}$ defines a basis of $\wedge^{d} I / I^{2}$, we denote by $\left(y_{1} \wedge \cdots \wedge y_{d}\right)^{\vee}$ its dual basis. In the same way, we define a basis $\left(z_{1} \wedge \cdots \wedge z_{d}\right)^{\vee}$ of $\omega_{X / Z}$.

1.3 Lemma. We preserve the notation. We define a homomorphism $\alpha$ in the following diagram so that it is commutative.

$$
\begin{aligned}
& \operatorname{Ext}_{R_{Y}}^{d}\left(R_{X}, \operatorname{Hom}_{R_{Z}}\left(R_{Y}, R_{Z}\right)\right) \longrightarrow \omega_{X / Z} \otimes_{R_{Y}} \operatorname{Hom}_{R_{Z}}\left(R_{Y}, R_{Z}\right)
\end{aligned}
$$

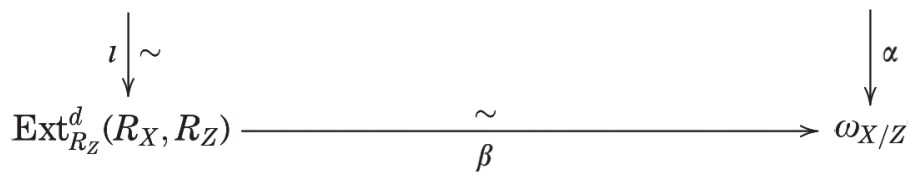

Here the horizontal isomorphisms are the isomorphisms of [Ha, III, 7.2]. Let $g^{*}\left(z_{i}\right)=\sum_{1 \leq j \leq d} f_{i j} y_{j}$ where $f_{i j} \in R_{Y}$. Here the expression may not be unique, but take one. We put $G:=\left(f_{i j}\right)_{i \leq i, j \leq d} \in \operatorname{Mat}_{d \times d}\left(R_{Y}\right)$. Then

$$
\alpha\left(\left(y_{1} \wedge \cdots \wedge y_{d}\right)^{\vee} \otimes \varphi\right)=\overline{\varphi(\operatorname{det}(G))} \cdot\left(z_{1} \wedge \cdots \wedge z_{d}\right)^{\vee}
$$

where the over-line denotes to take the image of the homomorphism $R_{Z} \rightarrow R_{X}$ inducing $h$.

Proof. On the way we prove the lemma, we will review the definition of the homomorphism $\beta$. Let $R_{Z} \zeta_{i}$ be a free $R_{Z}$-module of rank 1 whose gen- 
erator is $\zeta_{i}$. Let $K_{\bullet}:=\bigwedge^{\bullet}\left(\bigoplus_{i=1}^{d} R_{Z} \zeta_{i}\right)$ be the Koszul complex. By definition, the differential homomorphism $K_{r} \rightarrow K_{r-1}$ is defined by sending $\zeta_{i_{1}} \wedge \cdots \wedge \zeta_{i_{r}}$ to $\sum(-1)^{j} z_{i_{j}} \zeta_{i_{1}} \wedge \cdots \wedge \widehat{\zeta_{j}} \wedge \cdots \wedge \zeta_{i_{r}}$ where $\widehat{\zeta_{i_{j}}}$ means omit $\zeta_{i_{j}}$. The canonical homomorphism $R_{Z} \rightarrow R_{X}$ defines a complex

$$
\bigwedge^{\bullet}\left(\bigoplus_{i=1}^{d} R_{Z} \zeta_{i}\right) \rightarrow R_{X} \rightarrow 0,
$$

which is known to be a free resolution of $R_{X}$. Now, let $R_{Y} v_{i}$ be a free $R_{Y}$ module of rank 1 with basis $v_{i}$, and let us define a homomorphism

$$
\bigwedge^{r}\left(\bigoplus_{i=1}^{d} R_{Z} \zeta_{i}\right) \rightarrow \bigwedge^{r}\left(\bigoplus_{i=1}^{d} R_{Y} v_{i}\right)
$$

by mapping $\zeta_{i_{1}} \wedge \cdots \wedge \zeta_{i_{r}}$ to

$$
\left(\sum_{1 \leq j \leq d} f_{i_{1}, j} v_{j}\right) \wedge \cdots \wedge\left(\sum_{1 \leq j \leq d} f_{i_{r}, j} v_{j}\right)=\sum_{\left(j_{1}, \ldots, j_{r}\right) \in[1, d]^{r}} f_{i_{1}, j_{1}} \ldots f_{i_{r}, j_{r}} \cdot v_{j_{1}} \wedge \cdots \wedge v_{j_{r}}
$$

where $[1, d]$ is the set $\{i \in Z \mid 1 \leq i \leq d\}$. Then it is a standard calculation to check that these homomorphisms define a homomorphism of Koszul complexes:

$$
\gamma: \bigwedge^{\bullet}\left(\bigoplus_{i=1}^{d} R_{Z} \zeta_{i}\right) \rightarrow \bigwedge^{\bullet}\left(\bigoplus_{i=1}^{d} R_{Y} v_{i}\right) .
$$

This induces the following commutative diagram.

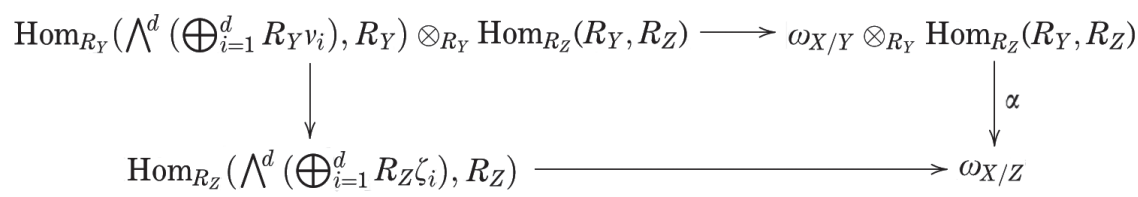

Here, the left vertical arrow is induced by $\gamma$. The bottom horizontal arrow is the surjective homomorphism defined by sending $\phi$ to $\overline{\phi\left(\zeta_{1} \wedge \cdots \wedge \zeta_{d}\right)} \cdot\left(z_{1} \wedge \cdots \wedge z_{d}\right)^{\vee}$. This factors through $\operatorname{Ext}_{R_{Z}}^{d}\left(R_{X}, R_{Z}\right)$, and this is $\beta$ by definition. The top horizontal arrow is defined in the same manner. The homomorphism $\gamma$ sends $\zeta_{1} \wedge \cdots \wedge \zeta_{d}$ to $\operatorname{det}(G) \cdot\left(v_{1} \wedge \cdots \wedge v_{d}\right)$. Thus, we get the lemma.

1.4. Now we will calculate the isomorphism $\mu_{X}$ in [Be2, Lemme 2.4.2], which is one of the two ingredients to calculate the Frobenius isomorphisms 
explicitly. The other ingredient is the explicit calculation of Frobenius by Garnier, which we will review in paragraph 2.2 .

Let us fix the situation and notation. We fix a positive integer $s>0$, and put $q:=p^{s}$ as in Notation. Let $S$ be a scheme endowed with quasicoherent $m$-PD ideal $(\mathfrak{a}, \mathfrak{b}, \alpha)$ such that $p \in a$ and $p$ is nilpotent, and $X$ be a smooth scheme over $S$ of relative dimension $d$. Let $S_{0}$ be the subscheme of $S$ defined by $a$, and $X_{0}:=X \times_{S} S_{0}$. Suppose $S$ and $X$ are affine schemes, and $X$ possesses a system of local coordinates $\left\{x_{i}\right\}_{1 \leq i \leq d}$ over $S$ (i.e. the homomorphism $X \rightarrow \mathrm{A}_{S}^{d}$ induced by $\left\{x_{i}\right\}$ is étale). Recall $X_{0}^{(s)}:=X_{0} \times_{S_{0}, F_{S_{0}}^{s}} S_{0}$ is the $s$-th relative Frobenius of $X_{0}$ over $S_{0}$. Let $X^{\prime}$ be a smooth lifting of $X_{0}^{(s)}$ over $S$. There exists a system of local coordinates $\left\{y_{i}\right\}_{1 \leq i \leq d}$ of $X^{\prime}$. Since $S$ and $X$ are affine, we may lift the relative Frobenius homomorphism denoted by $F: X \rightarrow X^{\prime}$ over $S$ uniquely such that $F^{*}\left(y_{i}\right)=x_{i}^{q}$.

We will use multi-index notation. For an integer $i$, we put $\underline{i}:=(i, \ldots, i)$ in $Z^{d}$. For $\underline{k}=\left(k_{1}, \ldots, k_{d}\right)$ and $\underline{k}^{\prime}=\left(k_{1}^{\prime}, \ldots, k_{d}^{\prime}\right)$ in $Z^{d}$, we denote by $\underline{k}<\underline{k}^{\prime}$ (resp. $\underline{k} \leq \underline{k}^{\prime}$ ) if $k_{i}<k_{i}^{\prime}$ (resp. $k_{i} \leq k_{i}^{\prime}$ ) for any $1 \leq i \leq d$. We define $\underline{k}-\underline{k}^{\prime}:=\left(k_{1}-k_{1}^{\prime}, \ldots, k_{d}-k_{d}^{\prime}\right)$.

We know that

$$
F_{*} \mathcal{O}_{X} \cong \bigoplus_{\underline{0} \leq \underline{k}<\underline{q}} \mathcal{O}_{X^{\prime}} \underline{x \underline{k}} .
$$

Consider the dual $\mathcal{O}_{X}^{\vee}:=\operatorname{Hom}_{\mathcal{O}_{X^{\prime}}}\left(F_{*} \mathcal{O}_{X}, \mathcal{O}_{X^{\prime}}\right)$. We denote the dual basis of $\left\{\underline{x}^{\underline{k}}\right\}$ by $\left\{H \underline{x}^{-\underline{k}}\right\}$. The notation may seem a little strange, but this notation is used to be consistent with Garnier's calculation (cf. paragraph 2.2). For a quasi-coherent $\mathcal{O}_{X^{\prime}}$-module $\mathscr{L}$, we get

$$
\begin{aligned}
& F^{*}(\mathscr{C l})=\mathcal{O}_{X} \otimes_{\mathcal{O}_{X^{\prime}}} \mathscr{C b} \\
& F^{b}(\mathscr{C b})=\operatorname{Hom}_{\mathcal{O}_{X^{\prime}}}\left(F_{*} \mathcal{O}_{X}, \mathscr{C b}\right) \cong \mathscr{C} \otimes_{\mathcal{O}_{X^{\prime}}} \mathcal{O}_{X}^{\vee}
\end{aligned}
$$

as $\mathcal{O}_{X}$-modules. We identify them, and a section $m \otimes \varphi$ of $\mathscr{\mathscr { C }} \otimes_{\mathcal{O}_{X^{\prime}}} \mathcal{O}_{X}^{\vee}$ is considered to be a section of $F^{b}(\mathscr{C l})$.

1.5 Proposition. We preserve the notation, in particular $S, X, X^{\prime}$ are affine. Recall the isomorphism of Berthelot [Be2, 2.4.2]

$$
\mu_{X}: \omega_{X} \stackrel{\sim}{\rightarrow} F^{b} \omega_{X^{\prime}}
$$

Using (1.4.1), we can described this isomorphism by

$$
\mu_{X}\left(d x_{1} \wedge \cdots \wedge d x_{d}\right)=\left(d y_{1} \wedge \cdots \wedge d y_{d}\right) \otimes H \underline{x}^{-(q-1)} .
$$


Proof. Before starting the proof, we remind that Conrad pointed out in [Co] that with sign convention of [Ha], many compatibilities stated in [Ha] do not hold, and we need to use the modified convention as in [Co, 2.2]. In this proof, since the outcome does not change, we follow the conventions of [Ha]. For skeptical readers, we put signs (1) through (4) below arrows of the homomorphisms whose sign change if we use the conventions of [Co], and see how they differ at the very end of this proof.

To avoid confusions, we put $Y:=X^{\prime}$ in this proof. Consider the following diagram.

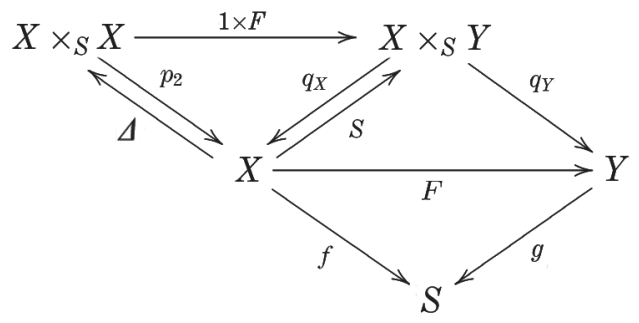

Here $p_{2}$ is the second projection, $\Delta$ is the diagonal morphism, $q_{X}$ is the first projection, $s$ is the graph morphism of $F, q_{Y}$ is the second projection, and $f$ and $g$ are structural morphisms. For an affine scheme $Z$, we denote the global sections of $Z$ by $R_{Z}$. Note that all the schemes appearing in the diagram are affine. Let us consider $X \times_{S} X\left(\right.$ resp. $\left.X \times_{S} Y\right)$ as a scheme over $X$ by the projection $p_{2}$ (resp. $q_{X}$ ) unless otherwise stated. We put

$$
\begin{array}{ll}
x_{1, i}:=x_{i} \otimes 1 & \text { in } R_{X \times_{S} X} \text { and } R_{X \times_{S} Y} \\
x_{\Delta, i}:=x_{i} \otimes 1-1 \otimes x_{i} & \text { in } R_{X \times_{S} X} \\
x_{s, i}:=F^{*}\left(y_{i}\right) \otimes 1-1 \otimes y_{i} & \text { in } R_{X \times_{S} Y} .
\end{array}
$$

The set $\left\{x_{\Delta, 1}, \ldots, x_{\Delta, d}, x_{1,1}, \ldots, x_{1, d}\right\} \quad$ (resp. $\left.\left\{x_{s, 1}, \ldots, x_{s, d}, x_{1,1}, \ldots, x_{1, d}\right\}\right)$ forms a system of local coordinates of $X \times_{S} X$ (resp. $X \times_{S} Y$ ), and $\left\{x_{\Delta, 1}, \ldots, x_{\Delta, d}\right\}$ (resp. $\left\{x_{s, 1}, \ldots, x_{s, d}\right\}$ ) defines a local system of parameters defining $\Delta(X)$ (resp. $s(X)$ ). We also note that $d x_{4, \bullet}:=d x_{\Delta, 1} \wedge \cdots \wedge d x_{\Delta, d}$ defines a basis of $\omega_{X \times_{S} X / X}$. For $\varphi \in \operatorname{Hom}_{R_{Y}}\left(R_{X}, R_{Y}\right)$, we denote by $\varphi^{\prime} \in \operatorname{Hom}_{R_{X \times S Y}}\left(R_{X \times \times_{S} X}, R_{X \times_{S} Y}\right)$ the homomorphism defined by $1 \otimes \varphi$. Now, let $d^{\prime}: R_{X} \rightarrow R_{X_{\times_{S}} X} \cong R_{X} \otimes_{R_{S}} R_{X}$ be a homomorphism of $R_{S}$-algebras defined by $d^{\prime}(b):=b \otimes 1-1 \otimes b$. We consider $R_{X \times_{S} X}$ as an $R_{X}$-algebra by the first component for a while. Then for $a, b \in R_{X}$, we get

$$
d^{\prime}(a b)=a d^{\prime}(b)+b d^{\prime}(a)-d^{\prime}(a) \cdot d^{\prime}(b) .
$$


Thus,

$$
\begin{aligned}
& (1 \times F)^{*}\left(x_{s, i}\right)=d^{\prime}\left(F^{*}\left(y_{i}\right)\right)=d^{\prime}\left(x_{i}^{q}\right)=\left(-d^{\prime} x_{i}^{q-1}+\sum_{q-1>j \geq 0} f_{i, j} d^{\prime} x_{i}^{j}\right) \cdot d^{\prime} x_{i} \\
& =: F_{i} \cdot x_{\Delta, i}
\end{aligned}
$$

with $f_{i, j} \in R_{X}$. By definition, we have

$$
\left\{\begin{array}{l}
\left(H x_{i}^{-(q-1)}\right)^{\prime}\left(d^{\prime} x_{j}^{k}\right)=0 \quad \text { for any } i \neq j \text { or } i=j \text { and } k \neq q-1 \\
\left(H x_{i}^{-(q-1)}\right)^{\prime}\left(d^{\prime} x_{i}^{q-1}\right)=-1 \otimes 1 .
\end{array}\right.
$$

Let

$$
G:=\operatorname{diag}\left(F_{1}, \ldots, F_{d}\right) \in \operatorname{Mat}_{d \times d}\left(R_{X \times_{S} X}\right)
$$

where diag denotes the diagonal matrix. Then, we obtain

$$
\begin{aligned}
\left(H \underline{x}^{-(\underline{q}-\underline{1})}\right)^{\prime}(\operatorname{det}(G))=\left(H \underline{x}^{-(\underline{q}-\underline{1})}\right)^{\prime}\left((-1)^{d} d^{\prime} x_{1}^{q-1}\right. & \left.\ldots d^{\prime} x_{d}^{q-1}\right)= \\
& (-1 \otimes 1)^{2 d}=1 \otimes 1 .
\end{aligned}
$$

We set back the convention, and consider $X \times_{S} X$ as a scheme over $X$ by $p_{2}$. The homomorphism $\mu_{X}$ is defined in the following way:

$$
\omega_{X} \cong f^{\sharp} \mathcal{O}_{S}[-d] \cong F^{b} g^{\sharp} \mathcal{O}_{S}[-d] \cong F^{b} \omega_{Y / S}
$$

where the first and third isomorphisms are by definition [Ha, III § 2] and the second isomorphism is induced by [Ha, III, Proposition 8.4]. In the rest of this proof, we will drop the section number III when we cite [Ha]. Since the sheaves we are considering are quasi-coherent and schemes are affine, we do not make any difference between sheaves and its global sections.

We will start to calculate from $F^{b} \omega_{Y / S}$. In the rest of this proof, we will use the identification $F^{D} \mathscr{C} \cong \mathcal{O}_{X}^{\vee} \otimes_{\mathcal{O}_{X^{\prime}}} \mathscr{C l}$ to describe the elements contrary to the standard convention (1.4.1) of this paper. Thus the sheaf $F^{\prime} \omega_{Y / S}$ is identified with $\operatorname{Hom}_{R_{Y}}\left(R_{X}, R_{Y}\right) \otimes_{R_{Y}} \omega_{Y / S}$. Take an element

$$
\varphi \otimes d y \bullet \in \operatorname{Hom}_{R_{Y}}\left(R_{X}, R_{Y}\right) \otimes_{R_{Y}} \omega_{Y / S} .
$$

First, we need to calculate the isomorphism $a: F^{b} \omega_{Y / S} \stackrel{\sim}{\rightarrow} s^{b} q_{Y}^{\sharp} \omega_{Y / S}$, which is the third isomorphism in the proof of [Ha, 8.4]. This isomorphism is the isomorphism of [Ha, 8.2]. To calculate this, first, we get an isomorphism

$$
\begin{aligned}
& \operatorname{Hom}_{R_{Y}}\left(R_{X}, R_{Y}\right) \otimes_{R_{Y}} \omega_{Y / S} \cong F^{b} \omega_{Y / S} \\
& \underset{\text { (1) }}{\stackrel{\sim}{b}} \Delta^{b} p_{2}^{\sharp} F^{b} \omega_{Y / S} \cong \omega_{X / X \times_{S} X} \otimes_{R_{X \times X}} \omega_{X \times_{S} X / X} \otimes_{R_{X}} \operatorname{Hom}_{R_{Y}}\left(R_{X}, R_{Y}\right) \otimes_{R_{Y}} \omega_{Y / S} .
\end{aligned}
$$


This isomorphism is defined in [Ha, 8.1]. Let $x_{\Delta, \bullet}^{\vee}$ denotes the dual basis of $x_{\Delta, 1} \wedge \cdots \wedge x_{\Delta, d}$ in $\omega_{X / X \times{ }_{S} X}, d y_{\bullet}$ denotes $d y_{1} \wedge \cdots \wedge d y_{d}$, and $d x_{1}$, denotes $d x_{1,1} \wedge \cdots \wedge d x_{1, d}$. We define $x_{s, \bullet}^{\vee}$ and $d x_{s, \bullet}$ in the same way. Then the isomorphism sends $\varphi \otimes d y_{\bullet}$ to $x_{\Delta, \bullet}^{\vee} \otimes d x_{1, \bullet} \otimes \varphi \otimes d y_{\bullet}$. Secondly, we get an isomorphism

$$
\begin{aligned}
\omega_{X / X \times_{S} X} \otimes_{R_{X \times X}} \omega_{X \times_{S} X / X} \otimes_{R_{X}} \operatorname{Hom}_{R_{Y}}\left(R_{X}, R_{Y}\right) \otimes_{R_{Y}} \omega_{Y / S} \\
\quad \cong \Delta^{b} p_{2}^{\sharp} F^{b} \omega_{Y / S} \stackrel{\sim}{\rightarrow} \Delta^{b}(1 \times F)^{b} q_{Y}^{\sharp} \omega_{Y / S} \\
\quad \cong \omega_{X / X \times_{S} X} \otimes_{R_{X \times X}} \operatorname{Hom}_{R_{X \times S} Y}\left(R_{X \times_{S} X}, R_{X \times_{S} Y}\right) \otimes_{R_{X \times Y}} \omega_{X \times_{S} Y / Y} \otimes_{R_{Y}} \omega_{Y / S} .
\end{aligned}
$$

This isomorphism is defined in [Ha, 6.3], and sends $x_{\Delta, \bullet}^{\vee} \otimes d x_{1, \bullet} \otimes \varphi \otimes d y \bullet$ to $x_{4, \bullet}^{\vee} \otimes \varphi^{\prime} \otimes d x_{1, \bullet} \otimes d y_{\bullet}$. Thirdly, we get an isomorphism

$$
\begin{aligned}
& \omega_{X / X \times \times_{S} X} \otimes \operatorname{Hom}_{R_{X \times S} Y}\left(R_{X \times \times_{S} X}, R_{X \times_{S} Y}\right) \otimes \omega_{X \times_{S} Y / Y} \otimes \omega_{Y / S} \cong \Delta^{b}(1 \times F)^{b} q_{Y}^{\sharp} \omega_{Y / S} \\
& \stackrel{\sim}{\rightarrow} s^{b} q_{Y}^{\sharp} \omega_{Y / S} \cong \omega_{X / X \times_{S} Y} \otimes_{R_{X \times S} Y} \omega_{X \times_{S} Y / Y} \otimes_{R_{Y}} \omega_{Y / S} .
\end{aligned}
$$

This isomorphism is defined in [Ha, 6.2], and this is the homomorphism we calculated in Lemma 1.3. Thus, using this lemma, we get that it sends $x_{\Delta, \bullet}^{\vee} \otimes \varphi^{\prime} \otimes d x_{1, \bullet} \otimes d y_{\bullet}$ to $\varphi^{\prime}(\operatorname{det}(G)) \cdot x_{s, \bullet}^{\vee} \otimes d x_{1, \bullet} \otimes d y_{\bullet}$ where $G$ is the matrix defined in (1.5.1). Combining these three isomorphisms we got, we obtain

$$
\begin{aligned}
a: \operatorname{Hom}_{R_{Y}}\left(R_{X}, R_{Y}\right) \otimes_{R_{Y}} \omega_{Y / S} & \stackrel{\sim}{\rightarrow} \omega_{X / X \times_{S} Y} \otimes_{R_{X \times S Y}} \omega_{X \times_{S} Y / Y} \otimes_{R_{Y}} \omega_{Y / S} \\
\varphi \otimes d y \bullet & \mapsto \overline{\varphi^{\prime}(\operatorname{det}(G))} \cdot x_{s, \bullet}^{\vee} \otimes d x_{1, \bullet} \otimes d y \bullet
\end{aligned}
$$

where the over-line denotes taking the image of the canonical homomorphism $R_{X \times_{S} Y} \rightarrow R_{X}$ inducing the morphism $s$.

Now, we come back to the definition of the isomorphism of [Ha, 8.4]. We need to calculate the isomorphism $b: s^{b} q_{Y}^{\sharp} \omega_{Y / S} \stackrel{\sim}{\rightarrow} s^{b} q_{X}^{\sharp} f^{\sharp} \mathcal{O}_{S}[-d]$, which is the second isomorphism in the proof of [Ha, 8.4]. We have an isomorphism

$$
\begin{aligned}
& s^{b} q_{Y}^{\sharp} \omega_{Y / S} \cong \omega_{X / X \times_{S} Y} \otimes_{R_{X \times S} Y} \omega_{X \times_{S} Y / Y} \otimes_{R_{Y}} \omega_{Y / S} \\
& \underset{\text { (2) }}{\sim} s^{b}\left(f \circ q_{X}\right)^{\sharp} \mathcal{O}_{S}[-d] \cong \omega_{X / X \times_{S} Y} \otimes_{R_{X \times S} Y} \omega_{X \times_{S} Y / S} .
\end{aligned}
$$

This isomorphism is defined in [Ha, 2.2], and sends $x_{s, \bullet}^{\vee} \otimes d x_{1, \bullet} \otimes d y \bullet$ to $x_{s, \bullet}^{\vee} \otimes\left((-1)^{d} d x_{s, \bullet} \wedge d x_{1, \bullet}\right)$. Then we get an isomorphism

$$
\begin{gathered}
\omega_{X / X \times_{S} Y} \otimes_{R_{X \times S} Y} \omega_{X \times_{S} Y / S} \cong \\
s^{b}\left(f \circ q_{X}\right)^{\sharp} \mathcal{O}_{S}[-d] \underset{\text { (3) }}{\stackrel{\sim}{\longrightarrow}} s^{b} q_{X}^{\sharp} f^{\sharp} \mathcal{O}_{S}[-d] \cong \omega_{X / X \times_{S} Y} \otimes \omega_{X \times_{S} Y / X} \otimes \omega_{X / S} .
\end{gathered}
$$


Explicit calculation of Frobenius isomorphisms and Poincaré duality etc. 101

This is also an isomorphism of [Ha, 2.2], and sends $x_{s, \bullet}^{\vee} \otimes\left(d x_{s, \bullet} \wedge d x_{1, \bullet}\right)$ to $(-1)^{d^{2}} x_{s, \bullet}^{\vee} \otimes d x_{s, \bullet} \otimes d x_{\bullet}$. Since $d^{2}+d$ is even, we get

$$
\begin{gathered}
b: \omega_{X / X \times_{S} Y} \otimes R_{X \times S}{ }_{\times_{X}} \omega_{X \times_{S} Y / Y} \otimes_{R_{Y}} \omega_{Y / S} \stackrel{\sim}{\rightarrow} \omega_{X / X \times_{S} Y} \otimes \omega_{X \times_{S} Y / X} \otimes \omega_{X / S} \\
x_{s, \bullet}^{\vee} \otimes d x_{1, \bullet} \otimes d y_{\bullet} \mapsto x_{s, \bullet}^{\vee} \otimes d x_{s, \bullet} \otimes d x_{\bullet} .
\end{gathered}
$$

At last, we get an isomorphism

$$
c: \omega_{X / X \times_{S} Y} \otimes \omega_{X \times_{S} Y / X} \otimes \omega_{X / S} \cong s^{b} q_{X}^{\sharp} \omega_{X / S} \underset{\text { (4) }}{\stackrel{\sim}{\rightarrow}} \omega_{X / S} .
$$

This isomorphism is defined in [Ha, 8.1], and sends $x_{s, \bullet}^{\vee} \otimes d x_{s, \bullet} \otimes d x$. to $d x$.

Now, by definition, $\mu_{X}^{-1}=c \circ b \circ a$. The above calculation shows that

$$
\mu_{X}^{-1}\left(\varphi \otimes d y_{\bullet}\right)=\overline{\varphi^{\prime}(\operatorname{det}(G))} \cdot d x_{\bullet} .
$$

Taking $\varphi=H \underline{x}^{-(\underline{q}-1)}$ and considering (1.5.2), we get the lemma.

As we noted at the beginning of this proof, we need some modification for the calculation of the homomorphisms (1) through (4) if we use the convention of [Co, 2.2]. Precisely for the homomorphisms (1) and (4), we need to multiply by $(-1)^{d(d-1) / 2}$, and for the homomorphisms (2) and (3), we need to multiply by $(-1)^{d^{2}}$. Thus, $\mu_{X}^{-1}$ is multiplied by $(-1)^{d(d-1)+2 d^{2}}=1$, and the result remains to be the same as we stated.

1.6 REMARK. When $F^{*}\left(y_{i}\right)$ is not equal to $x_{i}^{q}$, we can also calculate in the same way. We can write $d^{\prime}\left(F^{*}\left(y_{j}\right)\right)=\sum_{i} f_{i, j} \cdot d^{\prime} x_{i}$ where $f_{i, j} \in R_{X \times{ }_{S} X}$ using the notation of the proof of the proposition. We put $g_{i, j}:=$ $\left(\left(1 \otimes H \underline{x}^{q-1}\right)\left(f_{i, j}\right)\right)^{-}$where the over-line denotes to take the image of the canonical homomorphism $R_{X \times_{S} Y} \rightarrow R_{X}$. Using this, we get

$$
l\left(d x_{1} \wedge \cdots \wedge d x_{d}\right)=(\operatorname{det} \widetilde{G})^{-1}\left(H \underline{x}^{q-1}\right) d y_{1} \wedge \cdots \wedge d y_{d}
$$

where

$$
\widetilde{G}=\left(\begin{array}{ccc}
g_{1,1} & \ldots & g_{1, d} \\
\vdots & & \vdots \\
g_{d, 1} & \ldots & g_{d, d}
\end{array}\right)
$$

Note that this matrix is invertible since $\widetilde{G} \equiv I \bmod p \cdot \operatorname{Mat}\left(R_{X}\right)$.

Now the following theorem follows from the construction and Proposition 1.5 . 
1.7 THEOREM. We preserve the notation of paragraph 1.4, and let us denote by $\left(d x_{\bullet}\right)^{\vee}$ the dual basis of $d x_{\bullet}:=d x_{1} \wedge \cdots \wedge d x_{d}$ in $\omega_{X}^{-1}$, and the same for $d x^{\prime}$. and $\left(d x_{\bullet}^{\prime}\right)^{\vee}$. Let $\mathscr{l l}$ be a left $\mathscr{D}_{X^{\prime}}^{(m)}$-module, and $\mathscr{N}^{X}$ be a right $\mathscr{D}_{X^{\prime}}^{(m)}$ module. Recall two isomorphisms of Berthelot [Be2, 2.4.3, 2.4.4]:

$$
\begin{aligned}
& \mu_{\mathscr{L}}: \omega_{X} \otimes_{\mathcal{O}_{X}} F^{*} \mathscr{l l} \stackrel{\sim}{\rightarrow} F^{b}\left(\omega_{X^{\prime}} \otimes_{\mathcal{O}_{X^{\prime}}} \mathscr{C l}\right), \\
& v_{\mathscr{N}}: \omega_{X}^{-1} \otimes_{\mathcal{O}_{X}} F^{b} \cdot \mathscr{N} \stackrel{\sim}{\rightarrow} F^{*}\left(\omega_{X^{\prime}}^{-1} \otimes_{\mathcal{O}_{X^{\prime}}} \mathscr{N}\right) .
\end{aligned}
$$

Let $m \in \mathscr{l l}, m^{\prime} \in \mathscr{N}$, and $f \in \mathcal{O}_{X}$. Then we get that

$$
\begin{aligned}
& \mu_{\mathscr{N}}\left(d x_{\bullet} \otimes(f \otimes m)\right)=\left(d x_{\bullet}^{\prime} \otimes m\right) \otimes\left(H \underline{x}^{-(q-1)} \cdot f\right), \\
& v_{\mathscr{N}}\left(\left(d x_{\bullet}\right)^{\vee} \otimes\left(m^{\prime} \otimes\left(H \underline{x}^{-(\underline{q}-\underline{1})} \cdot f\right)\right)\right)=f \otimes\left(d x_{\bullet}^{\prime}\right)^{\vee} \otimes m^{\prime}
\end{aligned}
$$

by using the notation of (1.4.1).

\section{Explicit calculation of Frobenius isomorphisms}

In this section, we will give first applications of the theorem in the previous section. The main result of this section is the calculation of the Frobenius structure of push-forwards.

2.1. We will fix two situations for the basis $R$ often used in this paper.

1. The ring $R$ is complete discrete valuation ring as in Notation.

2. We moreover assume that the $s$-th absolute Frobenius isomorphism $F_{k}^{s}$ lifts to an automorphism $\operatorname{Spf}(R) \stackrel{\sim}{\rightarrow} \operatorname{Spf}(R)$ which is denoted by $\sigma$. In this case $k$ is automatically perfect.

For a scheme $X$ over $k$, we recall $X^{(s)}:=X \otimes_{k, F_{k}^{s *}} k$. Let $\mathscr{C}$ be a smooth formal scheme over $\operatorname{Spf}(R)$, and let $X_{0}$ the special fiber. Suppose that $X_{0}^{(s)}$ can be lifted to a smooth formal scheme $\mathscr{X}^{\prime}$ over $\operatorname{Spf}(R)$. In the situation 1 , we are able to consider Frobenius pull-backs even if there are no lifting of the relative Frobenius morphism $X_{0} \rightarrow X_{0}^{(s)}$ (cf. [Be2, 2.2.3]). Thus we are able to discuss the commutativity of Frobenius pull-backs with several cohomological operations such as push-forwards or duals etc. In the situation 2, moreover, we are able to define $F-\mathscr{D}_{\mathscr{X}, \mathrm{Q}}^{\dagger}$-modules (cf. [Be2, 4.5.1]).

2.2. We will review the second ingredient to calculate Frobenius isomorphisms, which are results of Garnier [Ga]. See [Ab] for another aspect of Garnier's result. 
Explicit calculation of Frobenius isomorphisms and Poincaré duality etc. 103

We will consider the situation 2.1.1. Let $\mathscr{B}$ be a smooth affine formal scheme over $R$ whose special fiber is denote by $X_{0}$, and suppose given a system of local coordinates $\left\{x_{1}, \ldots, x_{d}\right\}$. We denote by $\left\{\partial_{1}, \ldots, \partial_{d}\right\}$ the corresponding differential operators. For a positive integer $s$, we let $F_{k}^{s *}: k \rightarrow k$ be the $s$-th absolute Frobenius homomorphism. Let $\mathscr{f}^{\prime}$ be a smooth affine formal scheme over $R$ which is a lifting of $X_{0} \times_{\sigma_{s}} k$. The relative Frobenius morphism $X_{0} \rightarrow X_{0} \otimes_{F_{k}^{s *}} k$ can be lifted to a morphism $F_{\mathscr{C}}: \mathscr{C} \rightarrow \mathscr{C} \mathscr{C}^{\prime}$ by the universal property of smoothness since $\mathscr{C}$ is assumed to be affine. We sometimes denote $F_{\mathscr{C}}$ by $F$. We also fix a system of local coordinates $\left\{x_{1}^{\prime}, \ldots, x_{d}^{\prime}\right\}$ of $\mathscr{x}^{\prime}$ such that $F^{*}\left(x^{\prime}\right)=x^{q}$ using the universal property once again. We denote the corresponding differential operators by $\left\{\partial_{1}^{\prime}, \ldots, \partial_{d}^{\prime}\right\}$.

Garnier constructed in [Ga] a special differential operator $H \in \widehat{\mathscr{D}}_{\mathscr{X}}^{(s)}$ called the Dwork operator with the following properties:

1) Suppose a primitive $q$-th root of unity is contained in $R$. Then

$$
H_{i}=q^{-1} \sum_{\zeta^{q}=1} \sum_{k \geq 0}(\zeta-1)^{k} x_{i}^{k} \partial_{i}^{[k]}, \quad H:=\prod_{1 \leq i \leq d} H_{i} .
$$

These are global sections of $\widehat{\mathscr{D}}_{\mathscr{C}}^{(s)}$. The operator $H_{i}$ is called the Dwork operator corresponding to $x_{i}$. (cf. [Ga, Proposition 4.5.2])

2) The operator $H$ is a projector from $\mathcal{O}_{\mathscr{X}}$ to $\mathcal{O}_{\mathscr{X}^{\prime}}$. Precisely, we have $H^{2}=H$ in $\widehat{\mathscr{D}}_{\mathscr{X}}^{(s)}$, and its action on $\mathcal{O}_{\mathscr{X}}$ is $\mathcal{O}_{\mathscr{X}^{\prime}}$-linear.

(cf. [Ga, Proposition 2.5.1])

3) For $\underline{0} \leq \underline{k}<\underline{q}$, we get that $H \underline{x}^{-\underline{k}}$ in an element of $\widehat{\mathscr{D}}_{\mathscr{C}}^{(s)}$. We have $\sum_{0 \leq \underline{k}<\underline{q}} \underline{x}^{\underline{k}} H \underline{x}^{-\underline{k}}=1$.

(cf. [Ga, Proposition 2.5.1, 2.5.3])

4) For $\underline{0} \leq \underline{k}<q$, the operator $H \underline{x}^{-k}$ defines an $\mathcal{O}_{\mathscr{X}^{\prime}}$-linear homomorphism $\mathcal{O}_{\mathscr{X}} \rightarrow \mathcal{O}_{\mathscr{X}}$, and defines an element of $\mathcal{O}_{\mathscr{C}}^{\vee}$. The set $\left\{H \underline{x}^{-\underline{k}}\right\}$ defines the dual basis of $\left\{\underline{x}^{\underline{k}}\right\}$.

(cf. [Ga, Proposition 2.5.1, 2.5.3])

The property 4 justifies the notation $H \underline{x}^{-\underline{k}}$ as the dual basis used in paragraph 1.4. Now, we define

$$
\left(\partial_{i}^{\prime}\right)^{\circ}:=\left(q x^{q-1}\right)^{-1} \partial_{i} H,
$$

and $P:=\sum_{\underline{k}} f_{\underline{k}} \underline{\partial}^{\prime \underline{k}}$ in $\widehat{\mathscr{D}}_{\mathscr{O}^{\prime}}^{(m)}$ with $f_{\underline{k}} \in \mathcal{O}_{\mathscr{X}^{\prime}, \mathrm{Q}}$, we put

$$
P^{\circ}:=\sum_{\underline{k}} F^{*}\left(f_{\underline{k}}\right) \cdot\left(\underline{\partial^{\prime}}\right)^{\circ \underline{k}} .
$$


Note that $P^{\circ}$ is denoted by $P^{\prime}$ in [Ga, 4.6.1]. This defines a ring homomorphism $\widehat{\mathscr{D}}_{\mathscr{X}^{\prime}}^{(m)} \rightarrow \widehat{\mathscr{D}}_{\mathscr{K}}^{(m+s)} ; P \mapsto P^{\circ}$ (cf. [Ga, 4.3]).

The most important property of this operator is that with which we are able to describe the isomorphism [Be2, 4.1.2 (ii)]. Precisely, there exists the following canonical isomorphism

$$
F^{*} F^{b} \widehat{\mathscr{D}}_{\mathscr{C}^{\prime}}^{(m)} \cong \mathcal{O}_{\mathscr{X}} \otimes_{\mathcal{O}_{\mathscr{X}^{\prime}}} \widehat{\mathscr{D}}_{\mathscr{K}^{\prime}}^{(m)} \otimes_{\mathcal{O}_{\mathscr{X}^{\prime}}} \mathcal{O}_{\mathscr{X}}^{\vee} \stackrel{\sim}{\rightarrow} \widehat{\mathscr{D}}_{\mathscr{X}}^{(m+s)} .
$$

Then according to [Ga, 4.7.2], this isomorphism can be described as

$$
f \otimes P \otimes H \underline{x}^{-\underline{k}} \mapsto f \cdot P^{\circ} \cdot H \underline{x}^{-\underline{k}} .
$$

REMARK. Throughout [Ga], the residue field $k$ is assumed to be perfect (cf. [loc. cit. 1.1]). However, this assumption is not used in the paper, and this assumption is redundant. In fact, Berthelot is stating theorem of Frobenius descent $[\mathrm{Be} 2,4.2 .4]$ without posing any perfectness assumption.

2.3. Let $\mathscr{X}$ be as in the previous paragraph. Let $\mathscr{Y}$ be another smooth affine formal scheme over $R$ possessing a system of local coordinates $\left\{y_{1}, \ldots, y_{d^{\prime}}\right\}$. We also assume that we have a smooth lifting $\mathscr{Y}^{\prime}$ of the relative Frobenius with a system of local coordinates $\left\{y_{1}^{\prime}, \ldots, y_{d^{\prime}}^{\prime}\right\}$ and morphism $F_{\mathscr{Y}}: \mathscr{Y} \rightarrow \mathscr{Y}^{\prime}$ such that $F_{\mathscr{f}}^{*}\left(y_{i}^{\prime}\right)=y_{i}^{q}$ for any $i$. We fix one nonnegative integer $j$ and denote $\mathscr{C} \otimes R_{j}, \mathscr{Y} \otimes R_{j}, \mathscr{C}^{\prime} \otimes R_{j}, \mathscr{Y}^{\prime} \otimes R_{j}$ by $X, Y$, $X^{\prime}, Y^{\prime}$ respectively. Suppose given a morphism of special fibers $f_{0}: X_{0} \rightarrow Y_{0}$. Consider the following diagram.

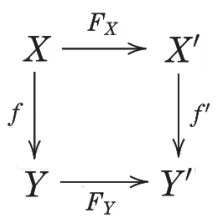

Here $F_{X}$ and $F_{Y}$ are reductions of $F_{\mathscr{X}}$ and $F_{\mathscr{Y}}$, and $f$ and $f^{\prime}$ are liftings of $f_{0}$ and $f_{0}^{\prime}$. In general, we are not able to take $f$ and $f^{\prime}$ so that the diagram is commutative. However, we can take $F_{X}$ and $F_{Y}$ locally with respect to $X$. To see this, it suffices to treat the case where $f$ is a closed immersion and smooth morphism individually, and in both cases, the verification is straightforward.

Let $\mathscr{C} b$ be a quasi-coherent $\mathcal{O}_{X^{\prime}}$-module. Since $X$ and $Y$ are affine schemes, we will identify quasi-coherent sheaves and its global sections. We list up conventions of identifications used to describe sections of certain sheaves as follows. 
Explicit calculation of Frobenius isomorphisms and Poincaré duality etc. 105

$$
\begin{array}{ll}
F_{X}^{b}(\mathscr{C}) \cong \mathscr{C} \otimes_{\mathcal{O}_{X^{\prime}}} \mathcal{O}_{X}^{\vee} \quad \mathscr{D}_{Y \rightarrow X}^{(m)} \cong \mathcal{O}_{X} \otimes_{\mathcal{O}_{Y}} \mathscr{D}_{Y}^{(m)} \\
F_{X}^{*}(\mathscr{C l}) \cong \mathcal{O}_{X} \otimes_{\mathcal{O}_{X^{\prime}}} \mathscr{\mathscr { C }} \quad \mathscr{D}_{Y \leftarrow X}^{(m)} \cong\left(\mathcal{O}_{X} \otimes_{\mathcal{O}_{Y}}\left(\mathscr{D}_{Y}^{(m)} \otimes_{\mathcal{O}_{Y}} \omega_{Y}^{-1}\right)\right) \otimes_{\mathcal{O}_{X}} \omega_{X}
\end{array}
$$

For example we have an identification

$$
F_{X}^{b} F_{Y^{\prime}}^{*} \mathscr{D}_{Y^{\prime} \leftarrow X^{\prime}}^{(m)} \cong \mathcal{O}_{Y} \otimes_{\mathcal{O}_{Y^{\prime}}}\left(\left(\mathcal{O}_{X^{\prime}} \otimes_{\mathcal{O}_{Y^{\prime}}} \mathscr{D}_{Y^{\prime}}^{(m)} \otimes_{\mathcal{O}_{Y^{\prime}}} \omega_{Y^{\prime}}^{-1}\right) \otimes_{\mathcal{O}_{X}^{\prime}} \omega_{X^{\prime}}\right) \otimes_{\mathcal{O}_{X^{\prime}}} \mathcal{O}_{X}^{\vee},
$$

and for $f \in \mathcal{O}_{Y}, g \in \mathcal{O}_{Y^{\prime}}, P \in \mathscr{D}_{Y^{\prime}}^{(m)}$ and $\underline{0} \leq \underline{k}<\underline{q}$, the section $f \otimes$ $\left(\left(g \otimes P \otimes\left(d y_{\bullet}^{\prime}\right)^{\vee}\right) \otimes d x_{\bullet}^{\prime}\right) \otimes H \underline{x}^{-\underline{k}}$ on the right side of the equality equally means a section on the left side by the identification.

2.4 Proposition. We preserve the notation of the previous paragraph. The inverse of the isomorphism of Berthelot [Be2, 3.4.2 (i)]

$$
\chi: F_{X}^{b} F_{Y}^{*} \mathscr{D}_{Y^{\prime} \leftarrow X^{\prime}}^{(m)} \stackrel{\sim}{\rightarrow} \mathscr{D}_{Y \leftarrow X}^{(m+s)}
$$

can be described in the following way using the identification of (2.3.1). Let $f \in \mathcal{O}_{X}$, and $P \in \mathscr{D}_{Y^{\prime}}^{(m)}$, then we get

$\chi\left(f \otimes\left(1 \otimes P \otimes\left(d y_{\bullet}^{\prime}\right)^{\vee} \otimes d x_{\bullet}^{\prime}\right) \otimes H \underline{x}^{-k}\right)=$

$$
x^{\underline{q}-\underline{k}-\underline{1}} \otimes\left(P^{\circ} \cdot H \underline{y}^{-(\underline{q}-\underline{1})} \cdot f\right) \otimes\left(d y_{\bullet}\right)^{\vee} \otimes d x_{\bullet} .
$$

PRoof. Recall that we are identifying quasi-coherent sheaves and its global sections. By using $v^{-1}$ in Theorem 1.7, we get an isomorphism

$$
\mathcal{O}_{Y} \otimes_{\mathcal{O}_{Y^{\prime}}}\left(\mathscr{D}_{Y^{\prime}}^{(m)} \otimes \omega_{Y^{\prime}}^{-1}\right) \rightarrow\left(\mathscr{D}_{Y^{\prime}}^{(m)} \otimes_{\mathcal{O}_{Y^{\prime}}} \mathcal{O}_{Y}^{\vee}\right) \otimes \omega_{Y}^{-1}
$$

The theorem is saying that this sends $f \otimes P \otimes\left(d y_{\bullet}^{\prime}\right)^{\vee}$ to $\left(P \otimes\left(H \underline{y}^{-(\underline{q}-\underline{1})} \cdot f\right)\right) \otimes$ $\left(d y_{\bullet}\right)^{\vee}$. By using $\mu^{-1}$, we get

$$
\left(\mathscr{D} \otimes_{\mathcal{O}_{X^{\prime}}} \omega_{X^{\prime}}\right) \otimes_{\mathcal{O}_{X^{\prime}}} \mathcal{O}_{X}^{\vee} \rightarrow\left(\mathcal{O}_{X} \otimes_{\mathcal{O}_{X^{\prime}}} \mathscr{D}\right) \otimes \omega_{X},
$$

where $\mathscr{D}:=f^{\prime *}\left(\left(\mathscr{D}_{Y^{\prime}}^{(m)} \otimes \mathcal{O}_{Y}^{\vee}\right) \otimes \omega_{Y}^{-1}\right)$. For a section $D$ of $\mathscr{D}$, this homomorphism sends $\left(D \otimes d x_{\bullet}^{\prime}\right) \otimes H \underline{x}^{-\underline{k}}$ to $\left(x^{q-\underline{k}-\underline{1}} \otimes D\right) \otimes d x$. At last, there exists the following isomorphism

$$
\begin{aligned}
\mathcal{O}_{X} \otimes \mathcal{O}_{X^{\prime}} f^{\prime *}\left(\left(\mathscr{D}_{Y^{\prime}}^{(m)} \otimes \mathcal{O}_{Y}^{\vee}\right) \otimes \omega_{Y}^{-1}\right) \underset{(*)}{\stackrel{\sim}{\longrightarrow}} f^{*}\left(\mathcal{O}_{Y} \otimes\left(\mathscr{D}_{Y^{\prime}}^{(m)} \otimes \mathcal{O}_{Y}^{\vee}\right) \otimes \omega_{Y}^{-1}\right) \stackrel{\sim}{\rightarrow} \\
f^{*} \mathscr{D}_{Y}^{(m+s)} \otimes \omega_{Y}^{-1} .
\end{aligned}
$$

Here the first isomorphism follows from the commutativity of the diagram. According to Garnier's calculation, this sends $f \otimes 1 \otimes\left(\left(Q \otimes H y^{-\underline{k}}\right) \otimes\left(d y_{\bullet}\right)^{\vee}\right)$ to $f \otimes\left(Q^{\circ} H \underline{y}^{-\underline{k}}\right) \otimes\left(d y_{\bullet}\right)^{\vee}$. Combining these, we get the proposition. 
REMARK. We will describe shortly the way to calculate $\chi$ when the diagram in paragraph 2.3 is not commutative. In this case, suppose the integer $m$ satisfies the inequality $p^{m}>e /(p-1)$ where $e$ was the absolute ramification index of $R$. Under this condition, we may use the Taylor isomorphism of $[\mathrm{Be} 2,2.1 .5]$ to compare $F_{Y} \circ f$ and $f^{\prime} \circ F_{X}$. This isomorphism can be described in the following way. Let $f, f^{\prime}: X \rightarrow Y$ be two morphisms of smooth schemes whose reductions over $k$ are the same morphisms, and suppose that $Y$ possesses a system of local coordinates $\left\{y_{1}, \ldots, y_{d}\right\}$. Let

$$
T:=\sum_{\underline{k} \geq 0}\left(f^{\prime *}(\underline{y})-f^{*}(\underline{y})\right)^{\{\underline{k}\}_{(m)}} \otimes \partial^{\langle\underline{k}\rangle_{(m)}}
$$

in $f^{*} \mathscr{D}_{Y}^{(m)}$. This is defined since we have the assumption on $m$. Let $\mathscr{C l}$ be a $\mathscr{D}_{Y}^{(m)}$-module. Then we can check that the isomorphism $\tau_{f, f^{\prime}}: f^{\prime *} \mathscr{L l} \stackrel{\sim}{\rightarrow} f^{*} \mathscr{l b}$ sends $1 \otimes m$ to $T \otimes m$ for a section $m$ of $\mathscr{l l}$.

Now, when the diagram is not commutative, the calculation of $\chi$ goes well exactly in the same way except for $(*)$ in the proof of the proposition. We use this calculation of Taylor isomorphism to compute $(*)$.

2.5. We use the same notation as paragraph 2.3. As the first application of Proposition 2.4, we will calculate the isomorphism

$$
F_{Y}^{*} f_{+}^{\prime} \mathscr{L b} \stackrel{\sim}{\rightarrow} f_{+} F_{X}^{*} \mathscr{l l}
$$

concretely. This result is used in $[\mathrm{AM}]$ to calculate the Frobenius structure of geometric Fourier transform defined by Noot-Huyghe explicitly.

Let $\mathscr{C}$ be a $\mathscr{D}_{X^{\prime}}^{(m)}$-module. In the proof of [Be2, 3.4.4], the isomorphism

$$
\begin{aligned}
& \xi: F_{Y}^{*}\left(\mathscr{D}_{Y^{\prime} \leftarrow X^{\prime}}^{(m)} \otimes_{\mathscr{D}_{X^{\prime}}^{(m)}}^{L_{L}} \mathscr{M}\right):= \\
& f^{-1} \mathcal{O}_{Y} \otimes_{f^{\prime-1}} \mathcal{O}_{Y^{\prime}}\left(\mathscr{D}_{Y^{\prime} \leftarrow X^{\prime}}^{(m)} \otimes_{\mathscr{V}_{X^{\prime}}^{(m)}}^{\mathrm{L}} \mathscr{l}\right) \stackrel{\sim}{\rightarrow}\left(\mathscr{D}_{Y \leftarrow X}^{(m+s)} \otimes_{\mathscr{D}_{X}^{(m+s)}}^{\mathrm{L}} F_{X}^{*} \mathscr{C l}\right)
\end{aligned}
$$

is defined. Using the projection formula, the isomorphism (2.5.1) is nothing but $R f_{*}(\xi)$.

Proposition. Let $\xi_{0}:=\mathscr{H}_{0}(\xi)$. For $P \in \mathscr{D}_{Y^{\prime}}^{(m)}$ and $m \in \mathscr{L}$, we have $\xi_{0}\left(\underline{y}^{\underline{l}} \otimes\left(1 \otimes P \otimes\left(d y^{\prime}\right)^{\vee} \otimes d x_{\bullet}^{\prime}\right) \otimes m\right)=$

$$
\left(1 \otimes\left(P^{\circ} \cdot H \underline{y}^{-(\underline{q}-1)} \cdot \underline{y}^{\underline{l}}\right) \otimes\left(d y_{\bullet}\right)^{\vee} \otimes d x_{\bullet}\right) \otimes\left(\underline{x}^{\underline{q}}-\underline{1} \otimes m\right) .
$$

Proof. Let us review the definition of $\xi_{0}$. Tensoring both sides of $\xi_{0}$ with $f^{-1} F_{Y^{\prime}}^{b} \mathscr{D}_{Y^{\prime}}^{(m)}$, it is equivalent to defining an isomorphism

$$
\mathscr{D}_{Y^{\prime} \leftarrow X^{\prime}}^{(m)} \otimes_{\mathscr{D}_{X^{\prime}}^{(m)}} \mathscr{L} \stackrel{\sim}{\rightarrow} f^{-1} F_{Y^{b}}^{b} \mathscr{D}_{Y^{\prime}}^{(m)} \otimes_{f^{-1} \mathscr{D}_{Y}^{(m+s)}}\left(\mathscr{D}_{Y \leftarrow X}^{(m+s)} \otimes_{\mathscr{D}_{X}^{(m+s)}} F_{X}^{*} \mathscr{C l}\right)
$$


Explicit calculation of Frobenius isomorphisms and Poincaré duality etc. 107

by [Be2, 2.5.6]. We get an isomorphism

$$
\begin{aligned}
& \mathscr{D}_{Y^{\prime} \leftarrow X^{\prime}}^{(m)} \otimes_{\mathscr{D}_{X^{\prime}}^{(m)}} \mathscr{L} \stackrel{\sim}{\rightarrow} F_{X^{b}}^{b} \mathscr{D}_{Y^{\prime} \leftarrow X^{\prime}}^{(m)} \otimes_{\mathscr{D}_{X}^{(m+s)}} F_{X}^{*} \mathscr{\mathscr { L }} \stackrel{\sim}{\rightarrow} f^{-1} F_{Y^{\prime}}^{b} \mathscr{D}_{Y^{\prime}}^{(m)} \otimes \\
& F_{Y}^{*} F_{X^{b}}^{\mathscr{D}_{Y^{\prime} \leftarrow X^{\prime}}^{(m)} \otimes F_{X}^{*} \mathscr{C l}}
\end{aligned}
$$

where the first isomorphism is by [Be2, 2.5.7] and the second by [Be2, 2.5.6]. Combining this isomorphism with $\chi$, we get the isomorphism (2.5.2). By Proposition 2.4, we see that (2.5.2) sends $\left(1 \otimes P \otimes\left(d y_{\bullet}^{\prime}\right)^{\vee} \otimes d x_{\bullet}^{\prime}\right) \otimes m$ to

$$
\left(1 \otimes H \underline{y}^{-\underline{l}}\right) \otimes\left(x^{\underline{q}-\underline{k}-\underline{1}} \otimes\left(P^{\circ} \cdot H \underline{y}^{-(\underline{q}-\underline{1})} \cdot \underline{y}^{\underline{l}}\right) \otimes\left(d y_{\bullet}\right)^{\vee} \otimes d x_{\bullet}\right) \otimes\left(\underline{x}^{\underline{k}} \otimes m\right)
$$

where $\underline{k}$ and $\underline{l}$ denote any element in $\mathbb{N}^{d}$ such that $\leq \underline{q}-\underline{1}$, and the proposition follows.

2.6. We consider the situation 2.1.1. As another application of the explicit description, we will show a proper base change type result. For the most familiar statement, see paragraph 5.7. Let $m$ be an integer such that $p^{m}>e /(p-1)$ and $i$ be a non-negative integer. Consider the following cartesian diagram of smooth schemes over $R_{i}$

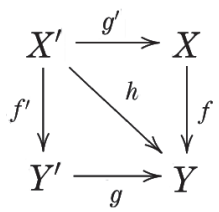

where $h=g \circ f^{\prime}$. Let $Z$ be one of $X, X^{\prime}, Y, Y^{\prime}$. We denote by $Z_{0}$ the reduction of $Z$ over $k$. We assume that $Z_{0}^{(s)}$ possesses a smooth lifting $\widetilde{Z}$ over $R_{i}$.

Under such situation, let us construct the following base change homomorphism:

$$
f^{\prime-1} \mathscr{D}_{Y^{\prime} \rightarrow Y}^{(m)} \otimes_{h^{-1} \mathscr{D}_{Y}^{(m)}}^{(m)} g^{\prime-1} \mathscr{D}_{Y \leftarrow X}^{(m)} \rightarrow \mathscr{D}_{Y^{\prime} \leftarrow X^{\prime}}^{(m)} \otimes_{\mathscr{D}_{X^{\prime}}^{(m)}}^{(m)} \mathscr{D}_{X^{\prime} \rightarrow X}^{(m)} \cdot
$$

For short, we denote $\mathscr{D}^{(m)}$ by $\mathscr{D}$. There exists a canonical homomorphism of rings $g^{-1} \mathscr{D}_{Y} \rightarrow \mathscr{D}_{Y^{\prime}}$. Thus, we get a homomorphism $h^{-1} \mathscr{D}_{Y} \otimes_{h^{-1} \mathcal{O}_{Y}} \omega_{X^{\prime} / Y^{\prime}} \stackrel{\alpha}{\rightarrow} f^{\prime-1} \mathscr{D}_{Y^{\prime}} \otimes_{\mathcal{O}_{Y^{\prime}}} \omega_{X^{\prime}} / Y^{\prime}$ of $\left(f^{\prime-1} \mathscr{D}_{Y^{\prime}}, g^{\prime-1} \mathscr{D}_{X}\right)$-bimodules where $\omega_{X^{\prime} / Y^{\prime}}:=\omega_{X^{\prime}} \otimes f^{\prime-1} \omega_{Y^{\prime}}$. We have the canonical section $1 \otimes 1$ in $\mathscr{D}_{X^{\prime} \rightarrow X} \cong \mathcal{O}_{X^{\prime}} \otimes_{f^{-1} \mathcal{O}_{X}} f^{-1} \mathscr{D}_{X}$. This section induces a homomorphism $f^{\prime-1} \mathscr{D}_{Y^{\prime}} \otimes_{\mathcal{O}_{Y^{\prime}}} \omega_{X^{\prime} / Y^{\prime}} \stackrel{\beta}{\rightarrow}\left(f^{\prime-1} \mathscr{D}_{Y^{\prime}} \otimes_{\mathcal{O}_{Y^{\prime}}} \omega_{X^{\prime} / Y^{\prime}}\right) \otimes_{\mathscr{D}_{X^{\prime}}} \mathscr{D}_{X^{\prime} \rightarrow X}$. Combining these, we get the homomorphism

$$
\beta \circ \alpha: h^{-1} \mathscr{D}_{Y} \otimes_{h^{-1} \mathcal{O}_{Y}} \omega_{X^{\prime} / Y^{\prime}} \rightarrow\left(f^{\prime-1} \mathscr{D}_{Y^{\prime}} \otimes_{\mathcal{O}_{Y^{\prime}}} \omega_{X^{\prime}} / Y^{\prime}\right) \otimes_{\mathscr{D}_{X^{\prime}}} \mathscr{D}_{X^{\prime} \rightarrow X}
$$


This induces the homomorphism (2.6.2) as required. Now consider the following diagram of cartesian squares:

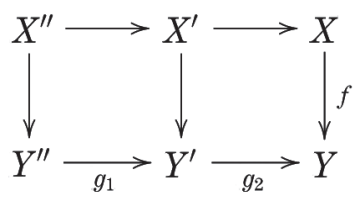

By definition, the following diagram of sheaves on $X^{\prime \prime}$, in which we omit pullbacks $f^{-1}$ etc., is commutative:

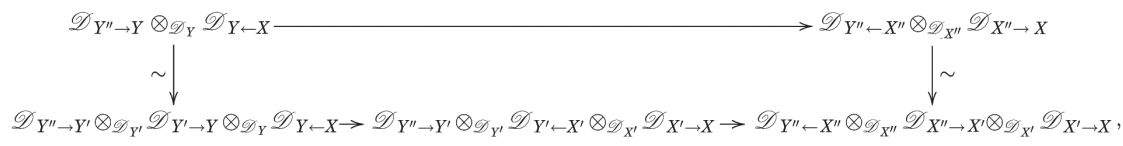

where horizontal homomorphisms are base change homomorphisms.

LEMma. Consider the diagram (2.6.1). We assume that $g$ is smooth. Then there is a canonical equivalence of functors

$$
g^{!} \circ f_{+} \cong f_{+}^{\prime} \circ g^{\prime !}: D_{\mathrm{qc}}^{b}\left(\mathscr{D}_{X}^{(m)}\right) \rightarrow D_{\mathrm{qc}}^{b}\left(\mathscr{D}_{Y^{\prime}}^{(m)}\right) .
$$

This equivalence is compatible with raising levels and Frobenius pullbacks. Consider the diagram (2.6.3) and assume that $g_{1}$ and $g_{2}$ are smooth. Then the equivalence is compatible with composition.

Proof. First, let us show that (2.6.2) induces a quasi-isomorphism

$$
f^{\prime-1} \mathscr{D}_{Y^{\prime} \rightarrow Y}^{(m)} \otimes_{h^{-1} \mathscr{D}}^{\mathrm{L}} g_{Y}^{(m)} g_{Y \leftarrow X}^{\prime-1} \mathscr{D}_{Y \leftarrow}^{(m)} \stackrel{\sim}{\rightarrow} \mathscr{D}_{Y^{\prime} \leftarrow X^{\prime}}^{(m)} \otimes_{\mathscr{D}_{X^{\prime}}^{(m)}}^{\mathrm{L}} \mathscr{D}_{X^{\prime} \rightarrow X}^{(m)} .
$$

By taking local coordinates, we can check that (2.6.2) is an isomorphism. Since $f^{\prime-1} \mathscr{D}_{Y^{\prime} \rightarrow Y}$ is a flat right $h^{-1} \mathscr{D}_{Y}$-module, we get a quasi-isomorphism

$$
f^{\prime-1} \mathscr{D}_{Y^{\prime} \rightarrow Y} \otimes_{h^{-1} \mathscr{D}_{Y}}^{\mathrm{L}} g^{\prime-1} \mathscr{D}_{Y \leftarrow X} \stackrel{\sim}{\rightarrow} f^{\prime-1} \mathscr{D}_{Y^{\prime} \rightarrow Y} \otimes_{h^{-1} \mathscr{D}_{Y}} g^{\prime-1} \mathscr{D}_{Y \leftarrow X} .
$$

From now on, we omit $f^{\prime-1}$ and so on, but we consider sheaves to be on $X^{\prime}$. Let us show that

$$
\mathscr{D}_{Y^{\prime} \leftarrow X^{\prime}}^{(m)} \otimes_{\mathscr{D}_{X^{\prime}}^{(m)}}^{\mathrm{L}} \mathscr{D}_{X^{\prime} \rightarrow X}^{(m)} \stackrel{\sim}{\rightarrow} \mathscr{D}_{Y^{\prime} \leftarrow X^{\prime}}^{(m)} \otimes_{\mathscr{D}_{X^{\prime}}^{(m)}} \mathscr{D}_{X^{\prime} \rightarrow X}^{(m)},
$$

or in other words $\mathscr{H}^{i}\left(\mathscr{D}_{Y^{\prime} \leftarrow X^{\prime}}^{(m)} \otimes_{\mathscr{D}_{X^{\prime}}^{(m)}}^{\mathrm{L}} \mathscr{D}_{X^{\prime} \rightarrow X}^{(m)}\right)=0$ for $i \neq 0$. When $m=0$, the argument is standard using the Spencer resolution $\mathscr{D}_{X^{\prime}}^{(0)} \otimes \Theta_{X^{\prime} / X}^{\bullet}$ of $\mathscr{D}_{X^{\prime} \rightarrow X}^{(0)}$ 
Explicit calculation of Frobenius isomorphisms and Poincaré duality etc. 109

where $\Theta_{X^{\prime} / X}$ denotes the relative tangent bundle of $X^{\prime} \rightarrow X$ (cf. [Be2, 4.3.1]). Let us see the general case. Since the verification is local, we may assume that the schemes are affine and $s=m$. We get

$$
\mathscr{D}_{Y^{\prime} \leftarrow X^{\prime}}^{(m)} \otimes_{\mathscr{D}_{X^{\prime}}^{(m)}}^{\mathrm{L}} \mathscr{D}_{X^{\prime} \rightarrow X}^{(m)} \cong F_{Y^{\prime}}^{*} F_{X}^{b}\left(\mathscr{D}_{\widetilde{Y}^{\prime} \leftarrow \widetilde{X}^{\prime}}^{(0)} \otimes_{\mathscr{D}_{\widetilde{X}^{\prime}}^{(0)}}^{\mathrm{L}} \mathscr{D}_{\widetilde{X}^{\prime} \rightarrow \widetilde{X}}^{(0)}\right)
$$

by [Be2, 2.5.6, 3.4.2]. This reduces the verification to the $m=0$ case, and the claim follows. Combining (2.6.2), (2.6.6), (2.6.7), we get the desired quasiisomorphism.

By construction, (2.6.2) is compatible with raising levels. Let us show the compatibility of Frobenius. We denote by relative Frobenius morphisms of the special fiber $X_{0}$ etc. by $F_{X}$ etc. Let us show that the following diagram is commutative where homomorphisms are quasi-isomorphisms.

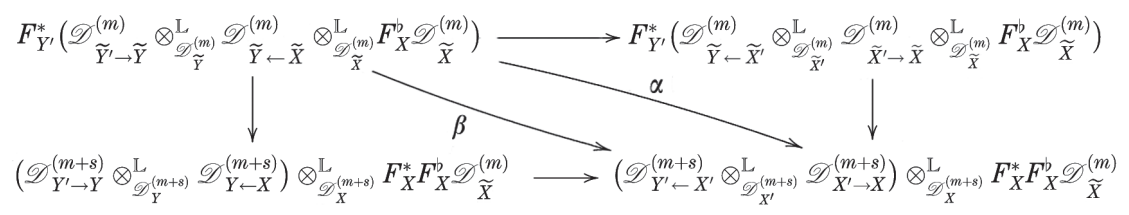

Let $\mathscr{C}$ be a left $\mathscr{D} \widetilde{X}_{\widetilde{m}}^{(m)}$-complex. By taking $\otimes_{\mathscr{D}_{X}^{(m+s)}}^{\mathrm{L}} F_{X}^{*} \mathscr{\mathscr { C }},(2.6 .8)$ induces the following commutative diagram:

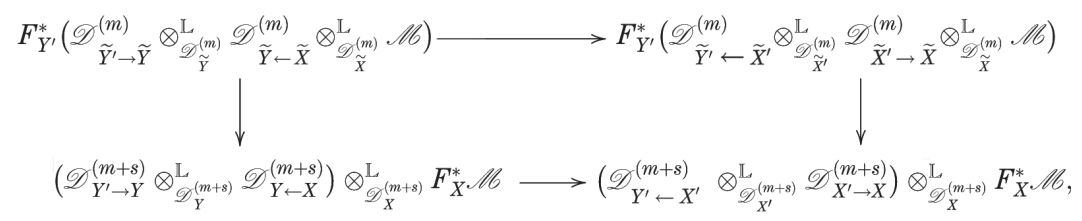

which implies the compatibility of Frobenius.

Let us prove the commutativity of (2.6.8). Since these complexes are concentrated in degree 0 , the problem is local on $X^{\prime}$. Thus, we may assume that any scheme possesses a system of local coordinates, and the following diagram is commutative.

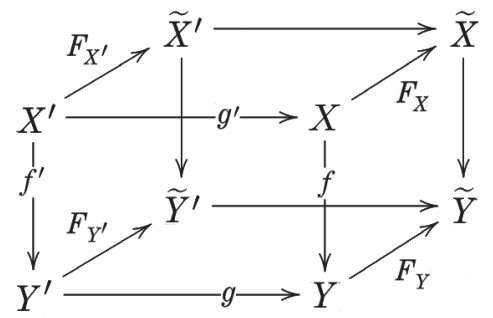


Let $\left\{\widetilde{t}_{i}\right\}_{1 \leq i \leq d_{X}}$ (resp. $\left\{t_{i}\right\}_{1 \leq i \leq d_{X}},\left\{\widetilde{s}_{j}\right\}_{1 \leq j \leq d_{Y}},\left\{s_{j}\right\}_{1 \leq j \leq d_{Y}}$ ) be a system of local coordinates of $\widetilde{X}$ (resp. $X, \widetilde{Y}, Y)$ such that $\left.F_{X}^{*}\left(\widetilde{t_{i}}\right\}\right)=t_{i}^{q}, F_{Y}^{*}\left(\widetilde{s_{j}}\right)=s_{j}^{q}$. As usual, we use the notation $d t_{\bullet}:=d t_{1} \wedge \cdots \wedge d t_{d_{X}}$ and so on. Let $H_{s_{j}}$ be the Dwork operator (cf. subsection 2.2) in $\mathscr{D}_{Y^{\prime}}^{(m+s)}$ corresponding to $s_{j}$, and we put $H_{s}:=\prod_{j=1}^{d_{Y}} H_{s_{j}}$. Let $x_{1}, \ldots, x_{d}$ be a local coordinate of $Y^{\prime}$ over $Y$. This can be seen also as a local coordinate of $X^{\prime}$ over $X$. We denote by $H_{i}$ the Dwork operator in $\mathscr{D}_{X^{\prime}}^{(m+s)}$ (resp. $\mathscr{D}_{Y^{\prime}}^{(m+s)}$ ) corresponding to $x_{i}$. We put $H_{x}:=\prod_{i=1}^{d} H_{i}$. Using the convention of (2.3.1), let

$$
\Xi:=1 \otimes\left((1 \otimes P) \otimes\left(1 \otimes 1 \otimes\left(d \widetilde{t}_{\bullet}\right)^{\vee} \otimes d \widetilde{s}_{\bullet}\right) \otimes(Q \otimes \phi)\right)
$$

be a section of $F_{Y^{\prime}}^{*}\left(\mathscr{D}_{\widetilde{Y^{\prime}} \rightarrow \widetilde{Y}}^{(m)} \otimes_{\mathscr{V}_{\widetilde{Y}}^{(m)}}^{\left(\mathscr{D}_{\widetilde{Y} \leftarrow \widetilde{X}}^{(m)} \otimes_{\mathscr{D}}(m)\right.} F_{X}^{b} \mathscr{D}_{\widetilde{X}}^{(m)}\right)$. To see the commutativity of (2.6.8), it suffices to show $\alpha(\Xi)=\beta(\Xi)$. Let $S:=\prod_{1 \leq i \leq d} x_{i}^{q-1} H_{i} x_{i}^{-(q-1)}$. By using Proposition 2.5,

$$
\begin{aligned}
& \alpha(\Xi)=\left(1 \otimes P^{\circ} \cdot H_{s} \underline{s}^{-(\underline{q}-1)} \cdot H_{x} \underline{x}^{-(\underline{q}-1)} \cdot f \otimes\left(d t_{\bullet}\right)^{\vee} \otimes d s_{\bullet}\right) \otimes\left(\underline{g}^{\underline{q}-1} \underline{x}^{\underline{q}-1} \otimes 1\right) \otimes(Q \otimes \phi) \\
& =\left(1 \otimes P^{\circ} \cdot H_{s} \underline{s}^{-(\underline{q}-\underline{1})} \cdot f \otimes\left(d t_{\bullet}\right)^{\vee} \otimes d s_{\bullet}\right) \otimes{ }^{t} S \cdot\left(\underline{q}^{\underline{q}} \underline{1} \otimes 1\right) \otimes(Q \otimes \phi) \\
& \beta(\Xi)=\left(1 \otimes P^{\circ} \cdot H_{s} \underline{s}^{-(\underline{q}-1)} \cdot f \otimes\left(d t_{\bullet}\right)^{\vee} \otimes d s_{\bullet}\right) \otimes\left(\underline{s}^{\underline{q}-1} \otimes 1\right) \otimes(Q \otimes \phi)
\end{aligned}
$$

To show that these two quantities are equal, it suffices to see that the image of ${ }^{t} S$ by the homomorphism $\mathscr{D}_{X^{\prime}}^{(m+s)} \rightarrow \mathscr{D}_{X^{\prime} \rightarrow X}^{(m+s)}$ is $1 \otimes 1$. To show this, it suffices to see that ${ }^{t} S(1)=1$ in $\mathcal{O}_{X^{\prime}}$. Since the claim is stable under base change, we may assume that $\zeta \in R_{i}$. By definition of $H$, it suffices to show that

$$
x_{i}^{-(q-1)} q^{-1} \sum_{\zeta^{q}=1} \sum_{k \geq 0}(\zeta-1)^{k} \partial_{i}^{[k]}\left(x_{i}^{k+q-1}\right)=1
$$

for any $1 \leq i \leq d$. The sum is equal to

$$
q^{-1} \sum_{\zeta^{q}=1} \sum_{k}(\zeta-1)^{k}\left(\begin{array}{c}
k+q-1 \\
k
\end{array}\right)=q^{-1} \sum_{\zeta^{q}=1} \sum_{k}(1-\zeta)^{k}\left(\begin{array}{c}
-q \\
k
\end{array}\right)=q^{-1} \sum_{\zeta^{q}=1} \zeta^{-q}=1,
$$

and the commutativity of (2.6.8) follows.

Let us complete the proof of the equivalence (2.6.5). We have

$$
\begin{aligned}
g^{!} \circ f_{+}(\mathscr{C l}) & :=\mathscr{D}_{Y^{\prime} \rightarrow Y} \otimes_{g^{-1} \mathscr{D}_{Y}}^{\mathrm{L}} g^{-1} R f_{*} \mathscr{D}_{Y \leftarrow X} \otimes_{\mathscr{D}_{X}}^{\mathrm{L}} \mathscr{C l} \\
& \cong R f_{*}^{\prime}\left(f^{\prime-1} \mathscr{D}_{Y^{\prime} \rightarrow Y} \otimes_{h^{-1} \mathscr{D}_{Y}}^{\mathrm{L}} g^{\prime-1}\left(\mathscr{D}_{Y \leftarrow X} \otimes_{\mathscr{D}_{X}}^{\mathrm{L}} \mathscr{C l}\right)\right) \\
& \stackrel{\sim}{\rightarrow} f_{*}^{\prime}\left(\mathscr{D}_{Y^{\prime} \leftarrow X^{\prime}} \otimes_{\mathscr{D}_{X^{\prime}}}^{\mathrm{L}} \mathscr{D}_{X^{\prime} \rightarrow X} \otimes_{g^{\prime-1} \mathscr{D}_{X}}^{\mathrm{L}} g^{\prime-1} \mathscr{C l}\right) \cong f_{+}^{\prime} \circ g^{\prime !}(\mathscr{C l}),
\end{aligned}
$$


where we used the flat base change in the first isomorphism, and the equivalence follows. The last statement follows by the commutativity of (2.6.4).

REMARK. When $g$ is a closed immersion, we can check that

$$
\mathscr{H}^{i}\left(f^{\prime-1} \mathscr{D}_{Y^{\prime} \rightarrow Y}^{(m)} \otimes_{h^{-1} \mathscr{D}}^{\mathrm{L}} g_{Y}^{(m)} g^{\prime-1} \mathscr{D}_{Y \leftarrow X}^{(m)}\right)=0, \quad \mathscr{H}^{i}\left(\mathscr{D}_{Y^{\prime} \leftarrow X^{\prime}}^{(m)} \otimes_{\mathscr{D}_{X^{\prime}}^{(m)}}^{\mathrm{L}} \mathscr{D}_{X^{\prime} \rightarrow X}^{(m)}\right)=0
$$

for $i \neq 0$. Thus, the base change homomorphism induces a quasi-isomorphism

$$
f^{\prime-1} \mathscr{D}_{Y^{\prime} \rightarrow Y}^{(m)} \otimes_{h^{-1} \mathscr{D}_{Y}^{(m)}}^{\mathbb{L}} g^{\prime-1} \mathscr{D}_{Y \leftarrow X}^{(m)} \stackrel{\sim}{\rightarrow} \mathscr{D}_{Y^{\prime} \leftarrow X^{\prime}}^{(m)} \otimes_{\mathscr{D}_{X^{\prime}}^{(m)}}^{\mathrm{L}} \mathscr{D}_{X^{\prime} \rightarrow X}^{(m)} .
$$

As in the proof of the previous lemma, this quasi-isomorphism defines a homomorphism of functors

$$
g^{!} \circ f_{+} \rightarrow f_{+}^{\prime} \circ g^{\prime !}: D_{\mathrm{qc}}^{b}\left(\mathscr{D}_{X}^{(m)}\right) \rightarrow D_{\mathrm{qc}}^{b}\left(\mathscr{D}_{Y^{\prime}}^{(m)}\right) .
$$

Moreover, by construction, we can see that this homomorphism coincides with that of Caro [Ca1, 2.2.18] when we take the limit and $g_{+}$. Thus, passing to the formal scheme, the homomorphism of functors becomes an equivalence and compatible with Frobenius if the resulting complexes on $Y^{\prime}$ is coherent by Berthelot-Kashiwara theorem [BeI, 5.3.3]. See Remark 5.7 (i) for more general situation.

2.7. Consider the situation in 2.1.1. Let us define the Tate twist (cf. [BeP, 2.3.8 (i)]). Let $\mathscr{O}$ and $\mathscr{H}$ are two smooth formal schemes, and suppose there exist smooth liftings $\mathscr{B}^{\prime}$ and $\mathscr{H}^{\prime}$ of $X_{0}^{(s)}$ and $Y_{0}^{(s)}$ where $X_{0}$ and $Y_{0}$ are special fibers of $\mathscr{C}$ and $\mathscr{H}$ as usual. Let $*$ be one of $\mathscr{C}, \mathscr{C}^{\prime}, \mathscr{H}, \mathscr{H}^{\prime}$, and $\mathcal{A}(*)$ be either $D_{\mathrm{coh}}^{b}\left(\mathscr{D}_{*, \mathrm{Q}}^{\dagger}\right)$ or ${\underset{L D}{\mathrm{Q}, \mathrm{qc}}}_{\left(\mathscr{D}_{*}\right.}^{b}\left(\widehat{\mathscr{D}}_{*}^{\bullet}\right)$. Let

$$
G: \mathcal{A}(\mathscr{C}) \rightarrow \mathcal{A}(\mathscr{Y}), \quad G^{\prime}: \mathcal{A}\left(\mathscr{C}^{\prime}\right) \rightarrow \mathcal{A}\left(\mathscr{Y}^{\prime}\right)
$$

be Q-linear functors. Now, suppose given a equivalence of functors

$$
\Psi: F_{\mathscr{Y}}^{*} \circ G^{\prime} \stackrel{\sim}{\rightarrow} G \circ F_{\mathscr{X}}^{*} .
$$

The triple $\left(G, G^{\prime}, \Psi\right)$ (we often abbreviate this as $(G, \Psi)$ or even $G$ if no confusion can arise) is called a cohomological functor with Frobenius isomorphism. The natural transform $\Psi$ is called the Frobenius isomorphism of the triple. Given $\left(G, G^{\prime}, \Psi\right)$ and an integer $d$, we define its Tate twist $\Psi(d)$ of the Frobenius isomorphism by $\Psi(d):=q^{-d} . \Psi$. We often denote by $G(d)$ the triple $\left(G, G^{\prime}, \Psi(d)\right)$ for simplicity. 
Now, we consider the situation 2.1.2. For a $\mathscr{D}_{\mathscr{X}, \mathrm{Q}}^{\dagger}$-module $\mathscr{C}$, we denote a $\mathscr{D}_{\mathscr{K}, Q}^{\dagger}$-module by $\mathscr{C}^{\sigma}$ the base change of $\mathscr{C}$ by using $\sigma$. Let $\mathscr{C}$ be an $F$ $\mathscr{D}_{\mathscr{X}, \mathrm{Q}}^{\dagger}$-module (resp. complex). By definition, this is a $\mathscr{D}_{\mathscr{K}, \mathrm{Q}}^{\dagger}$-module (resp. complex) equipped with an isomorphism ${ }^{1} \Phi: F^{*} \mathscr{L}^{\sigma} \stackrel{\sim}{\rightarrow} \mathscr{L}$. For any integer $d$, we define an $F$ - $\mathscr{D}_{\mathscr{C}}^{\dagger}$-module (resp. complex) $\mathscr{L}(d)$ called the Tate twist of $\mathscr{C}$ in the following way. The underlying $\mathscr{D}_{\mathscr{X}, Q}^{\dagger}$-module (resp. complex) is the same as that of $\mathscr{C}$. We denote by $\Phi^{\prime}$ the isomorphism $F^{*} \mathscr{C l}(d)^{\sigma} \rightarrow \mathscr{C}(d)$ induced by the Frobenius structure of $\mathscr{l} b$. The Frobenius structure $\Phi(d): F^{*} \mathscr{L}(d)^{\sigma} \rightarrow \mathscr{C}(d)$ of $\mathscr{L}(d)$ is by definition $q^{-d} \Phi^{\prime}$. Now, let $(G, \Psi)$ be a cohomological functor with Frobenius isomorphism. Then we get that $G(\mathscr{C})$ is naturally equipped with Frobenius structure, and we get for any integer $d$ that $G(d)(\mathscr{C l}) \cong G(\mathscr{C l})(d) \cong G(\mathscr{L}(d))$.

Let $(\mathscr{C l}, \Phi)$ and $(\mathscr{N}, \Psi)$ be two $F-\mathscr{D}_{\mathscr{C}, Q}^{\dagger}$-modules, and $\varphi: \mathscr{C l} \rightarrow \mathscr{N}$ be a homomorphism of $\mathscr{D}_{\mathscr{X}, \mathrm{Q}}^{\dagger}$-modules (where we do not consider the Frobenius structures). Consider the following diagrams where the left diagram is that of modules (or sheaves of modules):
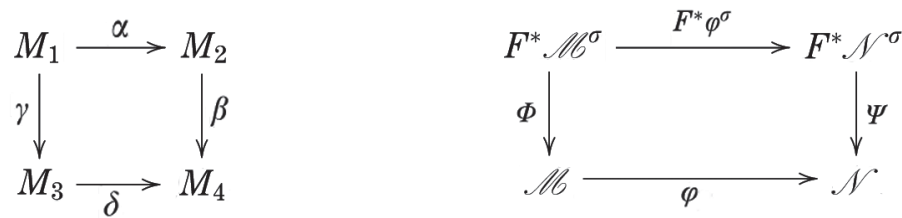

First, pay attention to the left diagram. Let $n$ be a rational number. We say that the diagram is commutative up to multiplication by $n$ if $n \cdot(\beta \circ \alpha)=$ $\delta \circ \gamma$ holds. Now, changing the attention to the right diagram, suppose that the diagram is commutative up to multiplication by $q^{d}$. Then we get that $\varphi$ defines a homomorphism $\mathscr{C}(d) \rightarrow \mathscr{N}$ as $F$ - $\mathscr{D}_{\mathscr{O}, \mathrm{Q}}^{\dagger}$-modules.

\section{Extraordinary pull-back and duality}

In this section, we prove a commutation result of the extraordinary pullback functor and the duality functor. The result can be seen as a part of a

${ }^{1}$ ) The definition of Frobenius structure here is slightly different from that

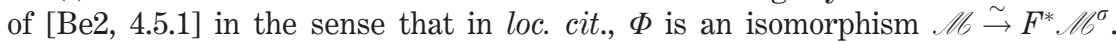
Since $\Phi$ is an isomorphism, it causes no difference. We adopted our definition to make it easier to see the compatibility with the definition of Frobenius structure of $F$-isocrystals. See also [loc. cit., Remarque 4.5.1]. 
"Poincare duality" in the theory of arithmetic $\mathscr{D}$-modules. For the explanation of this interpretation, see $\S 5$. By applying the commutation result, we get

$$
\mathrm{D}_{\mathscr{X}, Z}\left(\mathcal{O}_{\mathscr{X}, \mathrm{Q}}\left({ }^{\dagger} Z\right)\right) \cong \mathcal{O}_{\mathscr{K}, \mathrm{Q}}\left({ }^{\dagger} Z\right)(-d)
$$

where $\mathscr{X}$ is a smooth formal scheme, $Z$ is a divisor of its special fiber, and $d$ is the dimension of $\mathscr{C}$. Combining this result with a result of Caro, we are able to compare duality functors of arithmetic $\mathscr{D}$-modules and that of overconvergent isocrystals with Frobenius structures in a precise way. At the last part of this section, we compare the rigid cohomologies and the push-forwards in the theory of arithmetic $\mathscr{D}$-modules.

3.1. We consider the situation 2.1.1. Let $\mathscr{X}$ be a smooth formal scheme, and $Z$ be a divisor of its special fiber $X_{0}$. In this situation, we say that $(\mathscr{C}, Z)$ is a $d$-couple $e^{2}$ Let $(\mathscr{Y}, W)$ be another d-couple. A morphism of d-couples $f:(\mathscr{C}, Z) \rightarrow(\mathscr{Y}, W)$ is a morphism of special fibers $f_{0}: X_{0} \rightarrow Y_{0}$ such that $f\left(X_{0} \backslash Z\right) \subset Y_{0} \backslash W$, and $f^{-1}(W)$ is a divisor. A strict morphism of d-couples $f$ is a morphism $\widetilde{f}: \mathscr{X} \rightarrow \mathscr{Y}$ whose reduction on the special fiber is a morphism of d-couples. We say that the morphism $f_{0}$ (resp. $\widetilde{f}$ ) is the realization of the (resp. strict) morphism of d-couples $f$.

For a d-couple $(\mathscr{C}, Z)$, let us review the definition of the dual functor

$$
\mathrm{D}_{\mathscr{X}, Z}: D_{\text {perf }}^{b}\left(\mathscr{D}_{\mathscr{K}, Q}^{\dagger}\left({ }^{\dagger} Z\right)\right) \rightarrow D_{\text {perf }}^{b}\left(\mathscr{D}_{\mathscr{X}, Q}^{\dagger}\left({ }^{\dagger} Z\right)\right) \text {. }
$$

We note that there exists the canonical equivalence of categories

$$
D_{\text {perf }}^{b}\left(\mathscr{D}_{\mathscr{X}, \mathrm{Q}}^{\dagger}\left({ }^{\dagger} Z\right)\right) \stackrel{\sim}{\rightarrow} D_{\text {coh }}^{b}\left(\mathscr{D}_{\mathscr{L}, Q}^{\dagger}\left({ }^{\dagger} Z\right)\right)
$$

by [NH2, 3.2.3]. Let $d$ be the dimension of $\mathscr{C}$. For a perfect $\mathscr{D}_{\mathscr{C}, Q}^{\dagger}\left({ }^{\dagger} Z\right)$ complex $\mathscr{C}$, we define the functor $D_{\mathscr{C}, Z}$ by

$$
\mathrm{D}_{\mathscr{X}, Z}(\mathscr{C}):=R \mathcal{H o m}_{\mathscr{D}_{\mathscr{Q}, Q}^{\dagger}(Z)}\left(\mathscr{C}, \mathscr{D}_{\mathscr{X}, Q}^{\dagger}\left({ }^{\dagger} Z\right)\right) \otimes_{\mathcal{O}_{\mathscr{X}}} \omega_{\mathscr{X}}^{-1}[d] .
$$

For fundamental properties of this functor, see [Vi]. Here, we only note that this functor commutes with Frobenius pull-backs, and induces an equivalence between the derived categories of perfect complexes (with or without Frobenius structure).

Let $(\mathscr{C}, Z)$ and $(\mathscr{Y}, W)$ be d-couples, and let $f_{0}: X_{0} \rightarrow Y_{0}$ be a morphism such that $f_{0}\left(X_{0} \backslash Z\right) \subset Y_{0} \backslash W$. Assume that $f_{0}$ is smooth. Then $f_{0}^{-1}(W)$ is a

$\left({ }^{2}\right)$ This "d" stands for divisor. 
divisor, and in particular, $f_{0}$ induces a morphism of d-couples $f:(\mathscr{C}, Z) \rightarrow$ $(\mathscr{Y}, W)$. The functor $f_{0}^{!}: D_{\text {coh }}^{b}\left(\widehat{\mathscr{D}}_{\mathscr{Y}, \mathrm{Q}}^{(m)}(W)\right) \rightarrow D_{\mathrm{coh}}^{b}\left(\widehat{\mathscr{D}}_{\mathscr{X}, \mathrm{Q}}^{(m)}\left(f^{-1}(W)\right)\right)$ is defined in [Be2, 3.2.3 (ii)] and [BeI, 3.4.6]. By taking the inductive limit as [BeI, 4.3.3], we have the functor $f_{0}^{!}: D_{\mathrm{coh}}^{b}\left(\mathscr{D}_{\mathscr{Y}, \mathrm{Q}}^{\dagger}\left({ }^{\dagger} W\right)\right) \rightarrow D_{\mathrm{coh}}^{b}\left(\mathscr{D}_{\mathscr{X}, \mathrm{Q}}^{\dagger}\left({ }^{\dagger} f^{-1}(W)\right)\right)$. We define the functor

$$
f^{!}: D_{\mathrm{coh}}^{b}\left(\widehat{\mathscr{D}}_{\mathscr{Y}, \mathrm{Q}}^{(m)}(W)\right) \rightarrow D_{\mathrm{coh}}^{b}\left(\widehat{\mathscr{D}}_{\mathscr{K}, \mathrm{Q}}^{(m)}(Z)\right)
$$

by $(Z) \circ f_{0}^{!}$, where $(Z)$ denotes the functor tensoring with $\widehat{\mathscr{D}}_{\mathscr{X}, \mathrm{Q}}^{(m)}(Z)$. By taking the inductive limit, we also get a functor $f^{!}: D_{\operatorname{coh}}^{b}\left(\mathscr{D}_{\mathscr{Y}, \mathrm{Q}}^{\dagger}\left({ }^{\dagger} W\right)\right) \rightarrow$ $D_{\text {coh }}^{b}\left(\mathscr{D}_{\mathscr{Q}, Q}^{\dagger}\left({ }^{\dagger} Z\right)\right)$.

3.2. Let $m$ be an integer such that $p^{m}>e /(p-1)$ (cf. [Be2, A.4]). We denote $\mathcal{B}_{\mathscr{C}}^{(m)}(Z)$ and $\widehat{\mathcal{B}}_{\mathscr{K}}^{(m)}(Z)\left(\right.$ cf. [Be1, 4.2.4]) by $\mathcal{B}_{\mathscr{K}}^{(m)}$ and $\widehat{\mathcal{B}}_{\mathscr{C}}^{(m)}, \widehat{\mathcal{B}}_{\mathscr{C}}^{(m)} \widehat{\otimes} \widehat{\mathscr{D}}_{\mathscr{K}}^{(m)}$ by $\widetilde{\mathscr{D}}_{\mathscr{C}}^{(m)}$, and $\lim _{m} \widetilde{\mathscr{D}}_{\mathscr{X}}^{(m)}\left(=\mathscr{D}_{\mathscr{X}}^{\dagger}\left({ }^{\dagger} Z\right)\right)$ by $\widetilde{\mathscr{D}}_{\mathscr{X}}^{\dagger}$. We put $\widetilde{\omega}_{\mathscr{X}}:=\widehat{\mathcal{B}}_{\mathscr{X}}^{(m)} \widehat{\otimes}_{\mathcal{O}_{\mathscr{X}}} \omega_{\mathscr{X}} \cdot$ We denote by $D_{\mathrm{qc}}^{*}\left(\widetilde{\mathscr{D}}_{\mathscr{X}}^{(m)}\right)(* \in\{-, b\})$ the full subcategory of $D^{*}\left(\widehat{\mathscr{D}}_{\mathscr{K}}^{(m)}\right)$ consisting of quasi-coherent complexes (cf. [BeI, 3.2.1]), and $D_{\mathrm{Q}, q \mathrm{c}}^{*}\left(\widetilde{\mathscr{D}}_{\mathscr{X}}^{(m)}\right)$ by the category obtained by localizing $D_{\mathrm{qc}}^{*}\left(\widetilde{\mathscr{D}}_{\mathscr{X}}^{(m)}\right)$ with respect to isogenies (cf. [BeI, 3.3.2]). Finally, we denote by $D^{*}(\mathscr{X})(* \in\{+,-, b\})$ the derived category of $R$-modules on $\mathscr{C}$.

Lemma. Let $\mathscr{6}$ be a smooth formal scheme. Let 16 be a complex in $D_{\text {perf }}^{b}\left(\widetilde{\mathscr{D}}_{\mathscr{X}, \mathrm{Q}}^{(m)}\right)^{\mathrm{g}}$, and $\mathscr{N}$ be a complex in $D_{\mathrm{Q}, \mathrm{qc}}^{b}\left(\widetilde{\mathscr{D}}_{\mathscr{X}}^{(m)}\right)^{\mathrm{g}}$. Then the complex $\mathscr{L} \widehat{\otimes} \widehat{\mathcal{B}}_{x, Q}^{(m)} \mathscr{N}$ is bounded.

Proof. We will use the notation of SGA6 Exp. I. Since $D_{\text {perf }}^{b} \subset$ $D_{\text {ftd }}$ by 0.3 , we may assume that $\operatorname{parf-amp}(\mathscr{C l}) \subset[0, a]$. Let $n$ be an integer such that $\mathscr{H}^{i}(\mathscr{N})=0$ for $i<n$. It suffices to show that $\mathscr{H}^{i}\left(\mathscr{L} \widehat{\otimes}_{\widehat{B}_{x ; Q}^{(m)}}^{\mathrm{L}} \mathscr{N}\right)=0$ for $i<n-1$. Since this is local, and we may assume that $\mathscr{C}$ is affine. We will assume $\mathscr{C}$ to be affine in the following.

For a positive integer $r$, we say that a finitely generated $\widetilde{\mathscr{D}}_{\mathscr{C}}^{(m)}$-module $\mathscr{P}$ is $r$-nearly projective if there exists a $\widetilde{\mathscr{D}}_{\mathscr{C}}^{(m)}$-module $\mathcal{Q}$, an integer $b$, and a short exact sequence $0 \rightarrow \mathscr{P} \oplus \mathscr{Q} \rightarrow\left(\widetilde{\mathscr{D}}_{\mathscr{K}}^{(m)}\right)^{\oplus b} \rightarrow \mathscr{B} \rightarrow 0$ such that $\pi^{r} \mathscr{B}=0$. For any finitely generated projective $\widetilde{\mathscr{D}}_{\mathscr{L}, Q}^{(m)}$-module $\mathscr{P}^{\prime}$, there exists an integer $r$ and an $r$-nearly projective $\widetilde{\mathscr{D}}_{\mathscr{K}}^{(m)}$-module $\mathscr{P}$ such that $\mathscr{P} \otimes \mathrm{Q} \cong \mathscr{P}^{\prime}$. This shows that there exists a complex $\mathscr{P}$. of $r$-nearly projective $\widetilde{\mathscr{D}}_{\mathscr{C}}^{(m)}$-modules concentrated in $[0, a]$ such that $\mathscr{P} \bullet \otimes \mathrm{Q}$ is quasi-isomorphic to $\mathscr{C}$. Thus, it suffices to show that for any $r$-nearly 
Explicit calculation of Frobenius isomorphisms and Poincaré duality etc. 115 projective $\widetilde{\mathscr{D}}_{\mathscr{C}}^{(m)}$-module $\mathscr{P}$ and $\mathscr{N} \in D_{\mathrm{qc}}^{b}\left(\widetilde{\mathscr{D}}_{\mathscr{K}}^{(m)}\right)$ such that $\mathscr{H}^{i}(\mathscr{N})=0$ for $i<n$, we get

$$
\varpi^{r} \cdot \mathscr{H}^{i}\left(\mathscr{P} \widehat{\otimes}_{\widehat{\mathcal{B}}_{\mathscr{X}}^{(m)}}^{\mathbb{L}} \mathscr{N}\right)=0
$$

for $i<n-1$. Since $\mathscr{P}$ is $r$-nearly projective, $\varpi^{r} \mathscr{H}^{i}\left(\mathscr{P} \otimes_{\widehat{\mathcal{B}}_{\mathscr{C}}^{(m)}}^{\mathrm{L}} \mathscr{N}\right)=0$ for any $i<n$. This shows that

$$
\varpi^{r} \cdot \mathscr{H}^{i}\left(\mathscr{P}_{j} \otimes_{\mathcal{B}_{X_{j}}^{(m)}}^{\mathrm{L}} \mathscr{N}\right)=0
$$

for any $j$ and $i<n-1$, where $\mathscr{P}_{j}:=\mathscr{P} \otimes_{R} R_{j}$. Now, it remains to take $R l_{X_{*}}$, but since this functor is a right derived functor, we get the claim.

3.3. Now, let us state a key proposition in this section. Let $\mathscr{B}$ be a smooth formal scheme, and $\mathscr{C}^{\prime}$ be a smooth lifting of $X_{0}^{(s)}$.

Proposition. Let $\mathscr{M}$ be a complex in $D_{\text {perf }}^{b}\left(\widetilde{\mathscr{D}}_{\mathscr{X}, \mathrm{Q}}^{(m)}\right)^{\mathrm{g}}$, and $\mathscr{N}$ be a complex in $D_{\mathrm{Q}, \mathrm{qc}}^{b}\left(\widetilde{\mathscr{D}}_{\mathscr{C}}^{(m)}\right)^{\mathrm{g}}$. We denote $\mathrm{D}_{\mathscr{X}, Z}$ by $\mathrm{D}$. Then there exists the following quasi-isomorphism in $D^{+}(\mathscr{B})$

$$
\Psi: R \mathcal{H o m}_{\widetilde{\mathscr{D}}_{\mathscr{X}, Q}^{(m)}}\left(\widehat{\mathcal{B}}_{\mathscr{X}, Q}^{(m)}, \mathbb{D}(\mathscr{C l}) \widehat{\otimes}_{\widehat{\mathcal{B}}_{\mathscr{X}, Q}^{(m)}}^{\mathbb{L}} \mathscr{N}\right) \stackrel{\sim}{\rightarrow} R \mathcal{H o m}_{\widetilde{\mathscr{D}}_{\mathscr{X}, Q}^{(m)}}(\mathscr{C l}, \mathscr{N}) .
$$

Let $\mathscr{F}$ be a complex in $D_{\text {perf }}^{b}\left(\widetilde{\mathscr{D}}_{\mathscr{C}^{\prime}, \mathrm{Q}}^{(m)}\right)^{\mathrm{g}}$, and $\mathscr{G}$ be a complex in $D_{\mathrm{Q}, \mathrm{qc}}^{b}\left(\widetilde{\mathscr{D}}_{\mathscr{X}^{\prime}}^{(m)}\right)^{\mathrm{g}}$. Consider the following diagram:

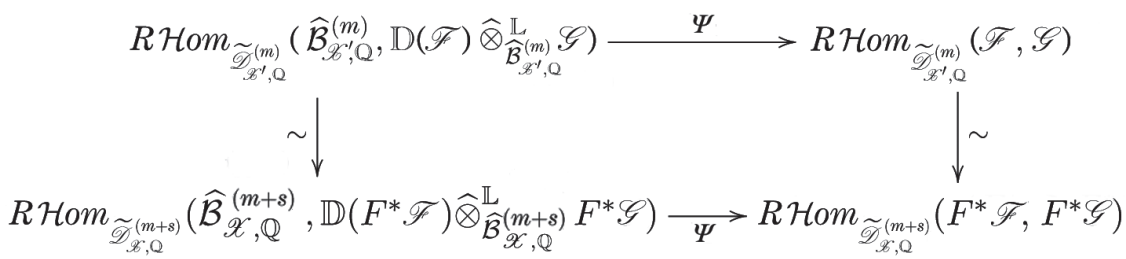

where the vertical homomorphisms are canonical isomorphisms of complexes which are defined by the theorem of Frobenius descent [Be2, 4.1.3]. This diagram is commutative up to multiplication by $q^{d}$ where d denotes the dimension of $\mathscr{X}$.

REMARK. We note that the complex $R \mathcal{H} m_{\widetilde{\mathscr{D}}_{\mathscr{B}, \mathrm{Q}}^{(m)}}\left(\widehat{\mathcal{B}}_{\mathscr{K}, \mathrm{Q}}^{(m)}, \mathrm{D}(\mathscr{C}) \widehat{\otimes}_{\widehat{\mathcal{B}}_{\mathscr{B}, Q}^{(m)}}^{\mathrm{L}} \mathscr{N}\right)$ makes sense thanks to Lemma 3.2.

The proof of the proposition will be given in paragraph 3.8, and we will start preparations of the proof from the next paragraph. Unless otherwise stated, $\mathscr{C}, \mathscr{N}, \mathscr{F}, \mathscr{G}$ are not the sheaves in the proposition. 
3.4. First, we will prove the following small lemma.

LEMma. Let $\mathscr{l l}$ be a complex in $D_{\mathrm{Q}, \mathrm{coh}}^{b}\left(\widetilde{\mathscr{D}}_{\mathscr{K}}^{(m)}\right)$ and $\mathscr{N}$ be one in $D_{\mathrm{Q}, \mathrm{qc}}^{b}\left(\widetilde{\mathscr{D}}_{\mathscr{X}}^{(m)}\right)$. Then, there is a canonical isomorphism

$$
\operatorname{Hom}_{D_{Q, q c}^{b}\left(\widetilde{\mathscr{Q}}_{\mathscr{X}}^{(m)}\right)}(\mathscr{l}, \mathscr{N}) \stackrel{\sim}{\rightarrow} \operatorname{Hom}_{\widetilde{\mathscr{X}}_{\mathscr{X}, Q}^{(m)}}(\mathscr{L l}, \mathscr{N}) .
$$

Proof. For $\mathscr{F}$ and $\mathscr{G}$ in $D_{\mathrm{Q}, \mathrm{qc}}^{b}\left(\widetilde{\mathscr{D}}_{\mathscr{C}}^{(m)}\right)$, we define

$$
R \mathcal{H o m}_{D_{\mathrm{Q}, \mathrm{c}}^{b}\left(\widetilde{\mathscr{V}}_{\mathscr{X}}^{(m)}\right)}(\mathscr{F}, \mathscr{G}):=\operatorname{RHom}_{\widetilde{\mathscr{D}}_{\mathscr{C}}^{(m)}(\mathscr{F}, \mathscr{G}) \otimes \mathbb{Q} .}
$$

There exists the canonical homomorphism

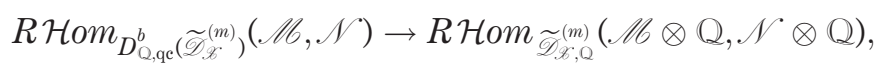

and it suffices to show that this is an isomorphism. Since the problem is local, we may assume that $\mathscr{X}$ is affine. It suffices to show the claim in the case where $\mathscr{\mathscr { C }}$ is projective. Since $\mathscr{\mathscr { C }}$ is a direct factor of $\left(\widetilde{\mathscr{D}}_{\mathscr{C}}^{(m)}\right)^{\oplus n}$ for some $n$, we are reduced to showing the case $\mathscr{\mathscr { C }}=\widetilde{\mathscr{D}}_{\mathscr{C}}^{(m)}$. In this case, the lemma is straightforward.

3.5. To compare Frobenius pull-backs, we need to construct a certain isomorphism in $D^{-}(\mathscr{C})$. Let. $\mathscr{N}$ be a complex in $D_{\text {perf }}^{b}\left(\widetilde{\mathscr{D}}_{\mathscr{X}^{\prime}, \mathrm{Q}}^{(m)}\right)^{\mathrm{d}}\left(\right.$ e.g. $\widetilde{\omega}_{\mathscr{O}^{\prime}, \mathrm{Q}}$ by (3.6.1)), and $\mathscr{C}$ be a complex in $D^{-}\left(\widetilde{\mathscr{D}}_{\mathscr{X}, \mathrm{Q}}^{(m)}\right)^{\mathrm{g}}$. The isomorphism we will construct is the following:

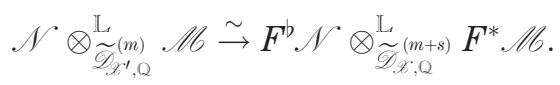

Let $\mathscr{Y}$ be a smooth formal scheme, and let $\mathscr{F}$ be a complex in $D_{\text {perf }}^{b}\left(\widetilde{\mathscr{D}}_{\mathscr{Y}, \mathrm{Q}}^{(m)}\right)^{\mathrm{d}}$, and $\mathscr{E}$ be a complex in $D^{-}\left(\widetilde{\mathscr{D}}_{\mathscr{Y}, \mathrm{Q}}^{(m)}\right)^{\mathrm{g}}$. First, there exists an isomorphism $D^{\mathrm{d}} \circ \mathbb{D}^{\mathrm{d}}(\mathscr{F}) \cong \mathscr{F}$ where

$$
\mathbb{D}^{\mathrm{d}}(\mathscr{T}):=R \mathcal{H o m}_{\widetilde{\mathscr{V}}_{\mathscr{y}, \mathrm{Q}}^{(m)}}\left(\mathscr{F}, \widetilde{\mathscr{V}}_{\mathscr{Y}, \mathrm{Q}}^{(m)}\right) \otimes \omega_{\mathscr{Y}}
$$

by [Vi, II, 3.6]. Using this, we get

$$
\begin{aligned}
\mathscr{F} \otimes_{\widetilde{\mathscr{V}}_{\mathscr{Y}, Q}^{(m)}}^{\mathrm{L}} \mathscr{E} & \cong R \mathcal{H o m}_{\widetilde{\mathscr{D}}_{\mathscr{Y}, \mathrm{Q}}^{(m)}}\left(\mathbb{D}^{\mathrm{d}}(\mathscr{F}), \widetilde{\mathscr{D}}_{\mathscr{Y}, \mathrm{Q}}^{(m)} \otimes \omega_{\mathscr{Y}}\right) \otimes_{\widetilde{\mathscr{V}}_{\mathscr{Y}, Q}^{(m)}}^{\mathrm{L}} \mathscr{E} \\
& \cong R \mathcal{H}_{\widetilde{\mathscr{V}}_{\mathscr{Y}, Q}^{(m)}}\left(\mathbb{D}^{\mathrm{d}}(\mathscr{F}), \omega_{\mathscr{Y}} \otimes \mathscr{E}\right)
\end{aligned}
$$

where the second isomorphism is by [Ca3, 2.1.17 (i)]. Now, we define (3.5.1) 
Explicit calculation of Frobenius isomorphisms and Poincaré duality etc. 117

in the following way:

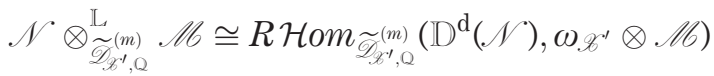

$$
\begin{aligned}
& \stackrel{\sim}{\rightarrow} R \mathcal{H o m}_{\widetilde{\mathscr{D}}_{\mathscr{X}, Q}^{(m+s)}}\left(F^{\mathrm{b}} \mathbb{D}^{\mathrm{d}}(\mathscr{N}), F^{\mathrm{b}}\left(\omega_{\mathscr{C}^{\prime}} \otimes \mathscr{\mathscr { C }}\right)\right)
\end{aligned}
$$

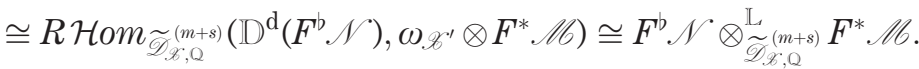

Here the second isomorphism follows by the theorem of Frobenius descent.

3.6. We will construct the homomorphism $\Psi$ in the proposition step by step. Let $\Theta_{\mathscr{C}}$ be the tangent sheaf on $\mathscr{C}$, and we put $\Theta_{\mathscr{C}}^{i}:=\bigwedge^{i} \Theta_{\mathscr{X}}$. First, note that there exists the Spencer resolution

$$
\widetilde{\mathscr{D}}_{\mathscr{X}, \mathrm{Q}}^{(m)} \otimes \Theta_{\mathscr{C}}^{\bullet} \rightarrow \widehat{\mathcal{B}}_{\mathscr{X}, \mathrm{Q}}^{(m)} \text {. }
$$

This can be seen in exactly the same way as the proof of [Be2, 4.3.3]. Indeed, $\mathcal{B}_{\mathscr{K}, \mathrm{Q}}^{(m)} \otimes \mathscr{D}_{\mathscr{K}, \mathrm{Q}}^{(m)}$ is flat over $\mathscr{D}_{\mathscr{K}, \mathrm{Q}}^{(m)}$. Since $\mathcal{B}_{\mathscr{K}}^{(m)} \otimes \mathscr{D}_{\mathscr{K}}^{(m)}$ is noetherian, the $p$-adic completion $\widetilde{\mathscr{D}}_{\mathscr{X}}^{(m)}$ is flat over $\mathcal{B}_{\mathscr{X}}^{(m)} \otimes \mathscr{D}_{\mathscr{C}}^{(m)}$. Thus, $\widetilde{\mathscr{D}}_{\mathscr{X}, \mathrm{Q}}^{(m)}$ is flat over $\mathscr{D}_{\mathscr{K}, \mathrm{Q}}^{(0)}$. It remains to show that $\widetilde{\mathscr{D}}_{\mathscr{K}, \mathrm{Q}}^{(m)} \otimes_{\mathscr{D} \mathscr{\mathscr { X }}, \mathrm{Q}}^{(0)} \mathcal{O}_{\mathscr{X}, \mathrm{Q}} \cong \widehat{\mathcal{B}}_{\mathscr{K}, \mathrm{Q}}^{(m)}$, whose proof is straightforward. This shows that $\widehat{\mathcal{B}}_{\mathscr{K}, Q}^{(m)}$ is perfect as a complex.

Let $\mathscr{C}$ be a bounded $\widetilde{\mathscr{D}}_{\mathscr{C}, \mathrm{Q}}^{(m)}$-complex. We have the following quasi-isomorphisms

$$
\begin{aligned}
& R \mathcal{H o m}_{\widetilde{\mathscr{D}}_{\mathscr{X}, Q}^{(m)}}\left(\widehat{\mathcal{B}}_{\mathscr{X}, \mathrm{Q}}^{(m)}, \mathscr{C l}\right) \\
& \cong R \mathcal{H o m}_{\widetilde{\mathscr{D}}_{\mathscr{X}, \mathrm{Q}}^{(m)}}\left(\widehat{\mathcal{B}}_{\mathscr{X}, \mathrm{Q}}^{(m)}, \widetilde{\mathscr{D}}_{\mathscr{X}, \mathrm{Q}}^{(m)}\right) \otimes \widetilde{\mathscr{D}}_{\mathscr{X}, \mathrm{L}}^{\mathrm{L}}(\mathrm{l}) \cong \widetilde{\omega}_{\mathscr{X}, \mathrm{Q}} \otimes \widetilde{\mathscr{D}}_{\mathscr{X}, \mathrm{L}}^{\mathrm{L}} \mathscr{L}[-d]
\end{aligned}
$$

where the first isomorphism holds by [Ca3, 2.1.17], and the second by (3.6.1) and [BeI, (3.5.5.1)]. Now the point where we need to use the explicit computation of Frobenius isomorphism of $\S 1$ is in the proof of the following lemma.

Lemma. Let $\mathscr{l l}$ be a complex in $D^{b}\left(\widetilde{\mathscr{D}}_{\mathscr{O}^{\prime}, \mathrm{Q}}^{(m)}\right)$. Consider the following diagram in $D^{b}(\mathscr{C})$.

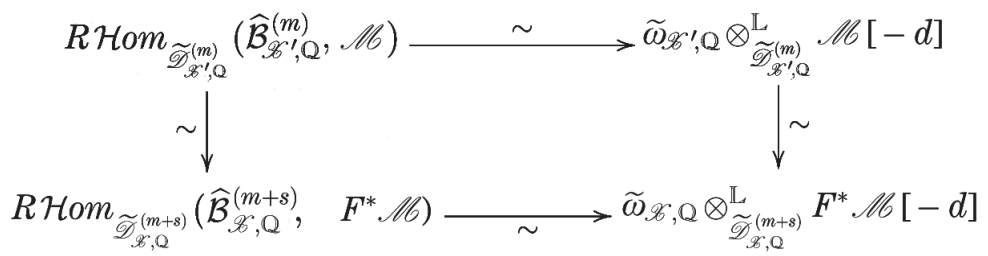


Here the right vertical homomorphism is (3.5.1) composed with the canonical isomorphism $F^{b} \omega_{\mathscr{O}} \cong \omega_{\mathscr{C}}$, and the left vertical homomorphism is induced by the functor $F^{*}$. Then this diagram is commutative up to multiplication by $q^{d}$.

Proof. Let $G$ be the functor $\mathcal{H o m}_{\widetilde{\mathscr{D}}_{\mathscr{8}, Q}^{(m+s)}}\left(F^{*} \widehat{\mathcal{B}}_{\mathscr{S}^{\prime}, \mathrm{Q}}^{(m)}, \bullet\right)$, and $G^{\prime}$ be the functor $\mathcal{H o m}_{\widetilde{\mathscr{D}}_{\mathscr{K}, \mathrm{Q}}^{(m+s)}}\left(\widehat{\mathcal{B}}_{\mathscr{K}, \mathrm{Q}}^{(m+s)}, \bullet\right)$. By (3.6.1), $R^{i} G$ and $R^{i} G^{\prime}$ vanishes for $i>d$. We define a functor $H$ to be $R^{d} G$, and $H^{\prime}$ to be $R^{d} G^{\prime}$. The canonical isomorphism $F^{*} \widehat{\mathcal{B}}_{\mathscr{K}^{\prime}, \mathrm{Q}}^{(m)} \stackrel{\sim}{\rightarrow} \widehat{\mathcal{B}}_{\mathscr{K}, \mathrm{Q}}^{(m+s)}$ induces an isomorphism of functors $H \stackrel{\sim}{\rightarrow} H^{\prime}$. By [Ha, I, 7.4], we get the following commutative diagram of functors.

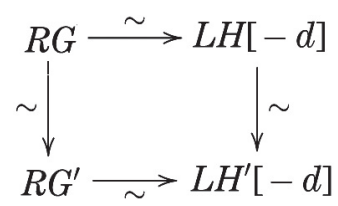

We note that flat $\widetilde{\mathscr{D}}_{\mathscr{K}, \mathrm{Q}}^{(m+s)}$-modules belong to the set $P$ of loc. cit. For a flat $\widetilde{\mathscr{D}}_{\mathscr{K}, \mathrm{Q}}^{(m+s)} L$-module $\mathscr{P}$, we get a canonical isomorphism $H(\mathscr{P}) \cong$ $F^{*} \widetilde{\omega}_{\mathscr{O}^{\prime}} \otimes_{\widetilde{D}_{\mathscr{X}}^{(m+s)}}^{(m)} \mathscr{P}$ and $H^{\prime}(\mathscr{P}) \cong \widetilde{\omega}_{\mathscr{C}} \otimes_{\widetilde{D}_{\mathscr{C}}^{(m+s)} \mathscr{P}}$, which induces canonical

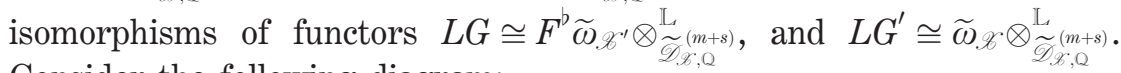
Consider the following diagram:

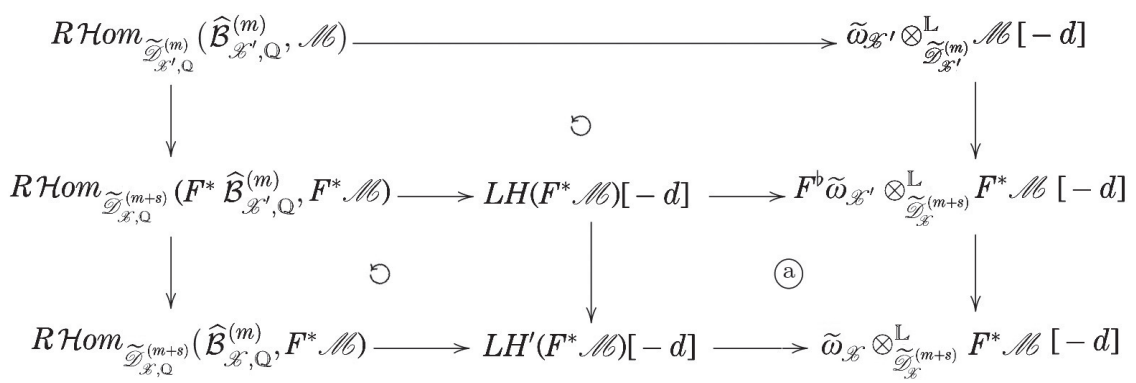

where all the arrows are isomorphisms, and $\bigcirc$ denotes that the marked square is commutative. Thus, to show that the big diagram is commutative up to some multiplication is equivalent to showing that the diagram (a) is commutative up to the same multiplication. Since the homomorphisms of the diagram (a) are induced by a diagram of functors between $H, H^{\prime}, F^{*} \widetilde{\omega}_{\mathscr{O}^{\prime}} \otimes, \widetilde{\omega}_{\mathscr{C}} \otimes$, it suffices to show the commutativity up to the same multiplication for this diagram of functors. Thus the problem is local. 
We may assume that $\mathscr{C}^{\prime}$ is affine, and possesses a system of local coordinates $\left\{y_{1}, \ldots, y_{d}\right\}$. Moreover, we can take a system of local coordinates $\left\{x_{1}, \ldots, x_{d}\right\}$ of $\mathscr{C}$ and a lifting $F: \mathscr{C} \rightarrow \mathscr{C}^{\prime}$ of relative Frobenius morphism such that $F^{*}\left(y_{i}\right)=x_{i}^{q}$. Under this situation, let us show that the diagram is commutative up to multiplication by $q^{d}$. From now on, we do not make any difference between quasi-coherent modules and its global sections. It suffices to show the commutativity in the case where $\mathscr{C}$ is flat over $\widehat{\mathscr{D}}_{\mathscr{O}^{\prime}, \mathrm{Q}}^{(m)}$.

Let $F_{*}: \Theta_{\mathscr{O}} \rightarrow F^{*} \Theta_{\mathscr{C}^{\prime}}$ be the canonical homomorphism. We have the following homomorphism $\widetilde{\mathscr{D}}_{\mathscr{X}, \mathrm{Q}}^{(m+s)} \otimes \Theta_{\mathscr{K}}^{k} \rightarrow F^{*}\left(\widetilde{\mathscr{V}}_{\mathscr{C}^{\prime}, \mathrm{Q}}^{(m)} \otimes \Theta_{\mathscr{X}^{\prime}}^{k}\right)$ sending $P \otimes\left(d y_{i_{1}} \wedge \cdots \wedge d y_{i_{k}}\right)$ to $P \cdot\left(1 \otimes q x_{i_{1}}^{q-1} \ldots q x_{i_{k}}^{q-1}\left(d x_{i_{1}} \wedge \cdots \wedge d x_{i_{k}}\right)\right)$. This defines, in fact, a homomorphism of complexes

$$
\widetilde{\mathscr{D}}_{\mathscr{K}, \mathrm{Q}}^{(m+s)} \otimes \Theta_{\mathscr{K}}^{\bullet} \rightarrow F^{*}\left(\widetilde{\mathscr{D}}_{\mathscr{K}^{\prime}, \mathrm{Q}}^{(m)} \otimes \Theta_{\mathscr{C}^{\prime}}^{\bullet}\right)
$$

by the proof of [Be2, 4.3.5]. It suffices to show that the diagram of modules

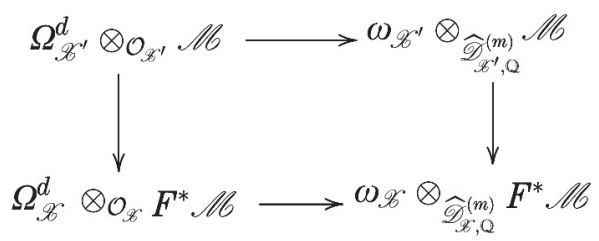

is commutative up to multiplication by $q^{d}$, where the left vertical homomorphism is induced by (3.6.3). Since the right vertical homomorphism sends $\left(d y_{1} \wedge \cdots \wedge d y_{d}\right) \otimes m$ to $x_{1}^{q-1} \ldots x_{d}^{q-1} \cdot\left(d x_{1} \wedge \cdots \wedge d x_{d}\right) \otimes(1 \otimes m)$ by using Proposition 1.5 and Theorem 1.7, we get the claim, and conclude the proof of the lemma.

3.7. We have the following lemma whose proof is similar to that of [Ca3, 2.1.27], and we leave it to the reader.

LEMma. Let $\mathscr{N}$ be a complex in $D_{\mathrm{qc}}^{-}\left(\widetilde{\mathscr{D}}_{\mathscr{C}}^{(m)}\right)^{\mathrm{d}}$, and $\mathscr{C l}$ and $\mathscr{C l}^{\prime}$ be two complexes in $D_{\mathrm{qc}}^{-}\left(\widetilde{\mathscr{D}}_{\mathscr{X}}^{(m)}\right)^{\mathrm{g}}$. Then there is a canonical isomorphism

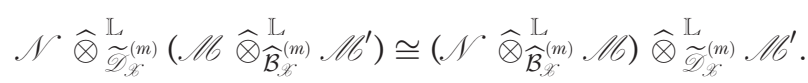

3.8. Proof of Proposition 3.3. Here, we use the notation in the proposition. We apply Lemma 3.6 to $\mathrm{D}(\mathscr{C}) \widehat{\otimes}_{\widehat{\mathcal{B}}_{x: Q}^{(m)}}^{\mathrm{L}}$. When we omit bases of tensor products, they are taken over $\widehat{\mathcal{B}}_{\mathscr{C}, Q}^{(m)}$. Then we get isomorphisms 


$$
\begin{aligned}
& R \mathcal{H o m}_{\widetilde{\mathscr{D}}_{\mathscr{X}, Q}^{(m)}}\left(\widehat{\mathcal{B}}_{\mathscr{K}, \mathrm{Q}}^{(m)}, \mathrm{D}(\mathscr{C l}) \widehat{\otimes}^{\mathrm{L}} \mathscr{N}\right) \cong \widetilde{\omega}_{\mathscr{X}, \mathrm{Q}} \otimes_{\widetilde{\mathscr{D}}_{\mathscr{P}, \mathrm{Q}}^{(m)}}^{\mathrm{L}}\left(\mathrm{D}(\mathscr{\mathscr { C }}) \widehat{\otimes}^{\mathrm{L}} \mathscr{N}\right)[-d]
\end{aligned}
$$

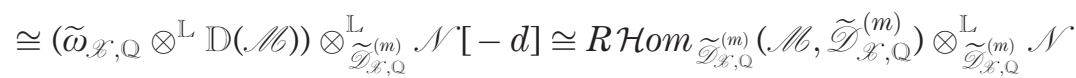

$$
\begin{aligned}
& \cong R \mathcal{H o m}_{\widetilde{\mathscr{D}}_{\mathscr{X}, Q}^{(m)}}(\mathscr{L}, \mathscr{N})
\end{aligned}
$$

where the first isomorphism is the one in paragraph 3.6, the second isomorphism is defined by Lemma 3.7, and we used the fact that $\mathscr{C}$ is a perfect complex in the last isomorphism (cf. [Ca3, 2.1.12, or 2.1.17]). This is nothing but $\Psi$ that we are looking for. The second, third, and the last isomorphisms are compatible with Frobenius. Thus the statement of Frobenius follows by Lemma 3.6.

3.9. Let $\mathscr{\mathscr { C }}$ be a smooth formal scheme. We denote by $\widetilde{D}_{\text {perf }}^{b}\left(\widetilde{\mathscr{D}}_{\mathscr{K}, Q}^{\dagger}\right)$ the full subcategory of $D_{\text {perf }}^{b}\left(\widetilde{\mathscr{D}}_{\mathscr{K}, \mathrm{Q}}^{\dagger}\right)$ consisting of a complex $\mathscr{C}$ such that there exists a complex $\mathscr{C}^{\prime}$ in $D_{\text {perf }}^{b}\left(\widetilde{\mathscr{D}}_{\mathscr{X}, \mathrm{Q}}^{(m)}\right)$ for some $m$ and an isomorphism $\widetilde{\mathscr{D}}_{\mathscr{X}, \mathrm{Q}}^{\dagger} \otimes \widetilde{\mathscr{D}}_{\mathscr{x}, \mathrm{Q}}^{(m)} \mathscr{\mathscr { C }}^{\prime} \cong \mathscr{\mathscr { C }}$.

LEMMA. Assume that $\mathscr{B}$ is quasi-compact. For any complex $\mathscr{C}$ in $D_{\text {coh }}^{b}\left(\widetilde{\mathscr{D}}_{\mathscr{X}, \mathrm{Q}}^{(m)}\right)$, there exists an integer $m^{\prime} \geq m$ such that $\widetilde{\mathscr{D}}_{\mathscr{C}, \mathrm{Q}}^{\left(m^{\prime}\right)} \otimes \widetilde{\mathscr{D}}_{\mathscr{X}, \mathrm{Q}}^{(m)} \mathscr{l l}$ is in $D_{\text {perf }}^{b}\left(\widetilde{\mathscr{D}}_{\mathscr{C}, \mathrm{Q}}^{\left(m^{\prime}\right)}\right)$. In particular, we have the canonical equivalence of categories

$$
\widetilde{D}_{\text {perf }}^{b}\left(\widetilde{\mathscr{D}}_{\mathscr{X}, \mathrm{Q}}^{\dagger}\right) \stackrel{\sim}{\rightarrow} D_{\text {perf }}^{b}\left(\widetilde{\mathscr{D}}_{\mathscr{K}, \mathrm{Q}}^{\dagger}\right) \stackrel{\sim}{\rightarrow} D_{\mathrm{coh}}^{b}\left(\widetilde{\mathscr{D}}_{\mathscr{K}, \mathrm{Q}}^{\dagger}\right) .
$$

Proof. Let us see the first claim. Since $\mathscr{B}$ is quasi-compact, the problem is local, and we may assume that $\mathscr{C}$ is affine. Since $\mathscr{C}$ is affine, we can take $\mathscr{C}$ to be a bounded complex such that each term is a coherent $\widetilde{\mathscr{D}}_{\mathscr{K}, Q}^{(m)}$-module. For $m^{\prime} \geq m$, we denote $\mathscr{L}^{\left(m^{\prime}\right)}:=\widetilde{\mathscr{D}}_{\mathscr{K}, \mathrm{Q}}^{\left(m^{\prime}\right)} \otimes \mathscr{\mathscr { C }}$ and $\mathscr{C l}^{\dagger}:=\widetilde{\mathscr{D}}_{\mathscr{C}, \mathrm{Q}}^{\dagger} \otimes \mathscr{\mathscr { C l }}$. Now, there exists a bounded finite locally projective $\widetilde{\mathscr{D}}_{\mathscr{O}, \mathrm{Q}}^{\dagger}$-complex $\mathscr{L}$ and a quasiisomorphism of complexes $\varphi: \mathscr{L} \rightarrow \mathscr{L}^{\dagger}$ since $\widetilde{\mathscr{D}}_{\mathscr{K}, \mathrm{Q}}^{\dagger}$ has finite Tor-dimension by the result of Noot-Huyghe in [NH2]. For a sufficiently large $m^{\prime}$, this complex can be descended to level $m^{\prime}$. Namely there exists a bounded locally finite projective $\widetilde{\mathscr{D}}_{\mathscr{C}}^{(m)}$-complex $\mathscr{L}^{\left(m^{\prime}\right)}$ and a homomorphism of complexes $\varphi^{\prime}: \mathscr{L}^{\left(m^{\prime}\right)} \rightarrow \mathscr{C}^{\left(m^{\prime}\right)}$ such that $\widetilde{\mathscr{D}}_{\mathscr{K}, Q}^{\dagger} \otimes \varphi^{\prime} \cong \varphi$. The homomorphism $\varphi^{\prime}$ may not be a quasi-isomorphism, but since the complexes are bounded and each term is coherent, there exists $m^{\prime \prime} \geq m^{\prime}$ such that the homomorphism $\widetilde{\mathscr{D}}_{\mathscr{K}, \mathrm{Q}}^{\left(m^{\prime \prime}\right)} \otimes \varphi^{\prime}$ becomes a quasi-isomorphism, which concludes the proof. The latter statement follows from [BeI, 4.2.4].

REMARK. We do not know if $\widetilde{D}_{\text {perf }}^{b}\left(\widetilde{\mathscr{D}}_{\mathscr{C}, \mathrm{Q}}^{\dagger}\right)$ and $D_{\text {perf }}^{b}\left(\widetilde{\mathscr{D}}_{\mathscr{X}, \mathrm{Q}}^{\dagger}\right)$ coincide or not in general. 
Explicit calculation of Frobenius isomorphisms and Poincaré duality etc. 121

3.10 TheOREM. Let $f:(\mathscr{C}, Z) \rightarrow(\mathscr{H}, W)$ be a morphism of $d$-couples whose realization is smooth. We assume that $X_{0}^{(s)}$ and $Y_{0}^{(s)}$ can be lifted to smooth formal schemes $\mathscr{O}^{\prime}$ and $\mathscr{Y}^{\prime}$. Let us denote by $d \geq 0$ the relative dimension of $X_{0}$ over $Y_{0}$. Then there is a canonical equivalence of cohomological functors from $\widetilde{D}_{\text {perf }}^{b}\left(\mathscr{D}_{\mathscr{Q}, Q}^{\dagger}\left({ }^{\dagger} W\right)\right)$ to $\left.\widetilde{D}_{\text {perf }}^{b}\left(\mathscr{D}_{\mathscr{X}, Q}^{\dagger}{ }^{\dagger} Z\right)\right)$ with Frobenius isomorphisms

$$
\left(\mathbb{D}_{\mathscr{X}, Z} \circ f^{!}\right)(d)[2 d] \stackrel{\sim}{\rightarrow} f^{!} \circ \mathbb{D}_{\mathscr{Y}, W} .
$$

Proof. Let $\mathscr{L}^{\dagger}$ be a complex in $\widetilde{D}_{\text {perf }}^{b}\left(\widetilde{\mathscr{D}}_{\mathscr{Y}, \mathrm{Q}}^{\dagger}\right)$. Then by definition, there exists $\mathscr{C}$ in $D_{\text {perf }}^{b}\left(\widetilde{\mathscr{D}}_{\mathscr{Y}, \mathrm{Q}}^{(m)}\right)$ for some $m$ and $\widetilde{\mathscr{D}}_{\mathscr{Y}, \mathrm{Q}}^{\dagger} \otimes_{\widetilde{D}_{\mathscr{Y}, Q}^{(m)}} \mathscr{C} \cong \mathscr{L}^{\dagger}$. First, let us define the homomorphism. By Proposition 3.3, we get a homomorphism

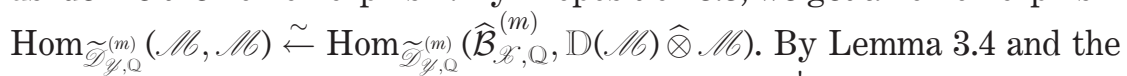
functoriality of the extraordinary pull-back functor $f$, we get

$$
\operatorname{Hom}_{\widetilde{\mathscr{D}}_{\mathscr{Y}, Q}^{(m)}}\left(\widehat{\mathcal{B}}_{\mathscr{X}, Q}^{(m)}, \mathrm{D}(\mathscr{C l}) \widehat{\otimes} \mathscr{\mathscr { C l }}\right) \rightarrow \operatorname{Hom}_{\widetilde{\mathscr{D}}_{\mathscr{X}, Q}^{(m)}}\left(f^{!} \widehat{\mathcal{B}}_{\mathscr{C}, Q}^{(m)}, f^{!}(\mathrm{D}(\mathscr{C l}) \widehat{\otimes} \cdot \mathscr{C l})\right) .
$$

This homomorphism is compatible with Frobenius pull-backs by the functoriality of the isomorphism [Be2, 3.2.4]. We get $f^{!} \widehat{\mathcal{B}}_{\mathscr{K}, \mathrm{Q}}^{(m)} \cong \widehat{\mathcal{B}}_{\mathscr{K}, \mathrm{Q}}^{(m)}\left[d_{f}\right]$. Moreover, $f^{!}(\mathbb{D}(\mathscr{C}) \widehat{\otimes} \mathscr{C l}) \cong f^{!} \circ \mathrm{D}(\mathscr{C l}) \widehat{\otimes} f^{!} \mathscr{C l}\left[-d_{f}\right]$. This isomorphism is also compatible with Frobenius pull-backs. Thus we get

$\operatorname{Hom}_{\widetilde{\mathscr{D}}_{\mathscr{X}, Q}^{(m)}}\left(f^{!} \widehat{\mathcal{B}}_{\mathscr{X}, \mathrm{Q}}^{(m)}, f^{!}(\mathbb{D}(\mathscr{C}) \widehat{\otimes} \mathscr{\mathscr { C }})\right) \cong \operatorname{Hom}_{\widetilde{\mathscr{D}}_{\mathscr{X}, Q}^{(m)}}\left(\widehat{\mathcal{B}}_{\mathscr{X}, \mathrm{Q}}^{(m)}\left[d_{f}\right], f^{!} \mathrm{D}(\mathscr{\mathscr { C }}) \widehat{\otimes} f^{!} \mathscr{\mathscr { C }}\left[-d_{f}\right]\right)$.

Now, using the proposition once again, we get an isomorphism

$\operatorname{Hom}_{\widetilde{\mathscr{D}}_{\mathscr{X}, Q}^{(m)}}\left(\widehat{\mathcal{B}}_{\mathscr{C}, \mathrm{Q}}^{(m)}\left[d_{f}\right], f^{!} \mathrm{D}(\mathscr{\mathscr { C }}) \widehat{\otimes} f^{!} \mathscr{\mathscr { C }}\left[-d_{f}\right]\right) \stackrel{\sim}{\rightarrow} \operatorname{Hom}_{\widetilde{\mathscr{D}}_{\mathscr{X}, Q}^{(m)}}\left(\mathbb{D} f^{!} \mathscr{\mathscr { C l }}, f^{!} \mathrm{D}(\mathscr{C l})\left[-2 d_{f}\right]\right)$.

Composing all of them, we obtain a homomorphism

$$
\operatorname{Hom}_{\widetilde{\mathscr{V}}_{\mathscr{Y}, Q}^{(m)}}(\mathscr{C l}, \mathscr{C l}) \rightarrow \operatorname{Hom}_{\widetilde{\mathscr{V}}_{\mathscr{B}, Q}^{(m)}}\left(\mathrm{D} f^{!} \mathscr{\mathscr { C }}, f^{!} \mathrm{D}(\mathscr{C l})\left[-2 d_{f}\right]\right) .
$$

The image of the identity is the homomorphism we wanted. By using [BeI, 4.3.3, 4.3.11], we get the following diagram.

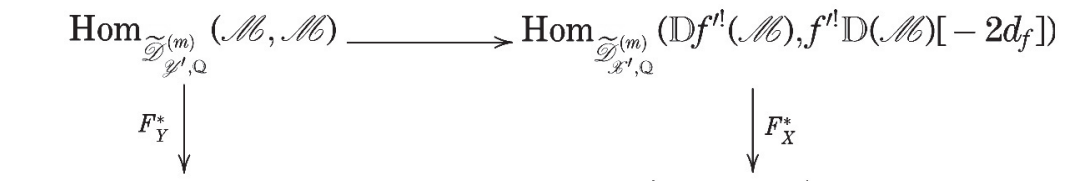

$$
\operatorname{Hom}_{\widetilde{\mathscr{V}}_{\mathscr{Y}, \mathrm{Q}}^{(m)}}\left(F_{Y}^{*} \mathscr{\mathscr { C }}, F_{Y}^{*} \mathscr{\mathscr { C l }}\right) \longrightarrow \operatorname{Hom}_{\widetilde{\mathscr{V}}_{\mathscr{X}, Q}^{(m)}}\left(\mathbb{D} f^{!}\left(F_{Y}^{*} \mathscr{\mathscr { C }}\right), f^{!} \mathbb{D}\left(F_{Y}^{*} \mathscr{\mathscr { C }}\right)\left[-2 d_{f}\right]\right)
$$

This diagram is commutative up to multiplication by $q^{-d_{Y}} \cdot q^{d_{X}}=q^{d_{f}}$ by the commutativity of Proposition 3.3. Thus we obtain the homomorphism 
$\mathrm{D} \circ f^{!}(\mathscr{C O})\left(d_{f}\right)\left[2 d_{f}\right] \rightarrow f^{!} \circ \mathrm{D}(\mathscr{C O})$. By tensoring with $\widetilde{\mathscr{D}}_{\mathscr{X}, \mathrm{Q}}^{\dagger}$, we get

$$
\mathrm{D} \circ f^{!}\left(\mathscr{\mathscr { C }} \mathrm{G}^{\dagger}\right)\left(d_{f}\right)\left[2 d_{f}\right] \rightarrow f^{!} \circ \mathrm{D}\left(\mathscr{\mathscr { C }} \mathrm{C}^{\dagger}\right)
$$

by using [BeI, 3.4.6 (iii)] and [Vi, I.5.4]. By construction, this does not depend on the choice of $\mathscr{L}$. It remains to show that this homomorphism is an isomorphism when $f$ is smooth.

It suffices to show the equality for $\mathscr{C}=\widetilde{\mathscr{D}}_{\mathscr{Y}, \mathrm{Q}}^{\dagger}$, and we can forget about Frobenius pull-backs. We get $R \mathcal{H} \operatorname{lom}_{\widetilde{\mathscr{D}}_{\mathscr{B}, \mathrm{Q}}^{\dagger}}\left(\widetilde{\mathscr{D}}_{\mathscr{O} \rightarrow \mathscr{Y}, \mathrm{Q}}^{\dagger}, \widetilde{\mathscr{D}}_{\mathscr{X}, \mathrm{Q}}^{\dagger}\right)\left[d_{f}\right] \cong \widetilde{\mathscr{D}}_{\mathscr{Y} \leftarrow \mathscr{X}, \mathrm{Q}}^{\dagger}$. Indeed,

$$
\begin{aligned}
& R \mathcal{H o m}_{\widetilde{\mathscr{D}}_{\mathscr{X}, \mathrm{Q}}^{\dagger}}\left(\widetilde{\mathscr{D}}_{\mathscr{C} \rightarrow \mathscr{Y}, \mathrm{Q}}^{\dagger}, \widetilde{\mathscr{D}}_{\mathscr{R}, \mathrm{Q}}^{\dagger}\right)\left[d_{f}\right] \cong R \mathcal{H o m}_{\mathscr{D}_{\mathscr{X}, \mathrm{Q}}^{\dagger}}\left(\mathscr{D}_{\mathscr{X}, \mathrm{Q}}^{\dagger} \otimes \Theta_{\mathscr{K} \mid \mathscr{Y}}^{\bullet}, \mathscr{D}_{\mathscr{X}, \mathrm{Q}}^{\dagger}\right)\left[d_{f}\right] \\
& \cong \Omega_{\mathscr{K} / \mathscr{Y}}^{\bullet} \otimes \widetilde{\mathscr{D}}_{\mathscr{X}, \mathrm{Q}}^{\dagger} \cong \widetilde{\mathscr{D}}_{\mathscr{Y} \leftarrow \mathscr{K}, \mathrm{Q}}^{\dagger} \text {. }
\end{aligned}
$$

Thus, we get

$$
\begin{aligned}
\mathbb{D} \circ f^{!}\left(\widetilde{\mathscr{D}}_{\mathscr{Y}, \mathrm{Q}}^{\dagger}\right)\left[2 d_{f}\right] \cong \mathbb{D}\left(\widetilde{\mathscr{D}}_{\mathscr{X} \rightarrow \mathscr{Y}, \mathrm{Q}}^{\dagger}\right)\left[d_{f}\right] \\
\quad \cong \widetilde{\mathscr{D}}_{\mathscr{Y} \leftarrow \mathscr{K}, \mathrm{Q}}^{\dagger} \otimes \omega_{\mathscr{X}}^{-1}\left[d_{f}\right] \cong f^{!} \widetilde{\mathscr{V}}_{\mathscr{Y}, \mathrm{Q}}^{\dagger} \otimes \omega_{\mathscr{Y}}^{-1} \cong f^{!} \circ \mathbb{D}\left(\widetilde{\mathscr{D}}_{\mathscr{Y}, \mathrm{Q}}^{\dagger}\right) .
\end{aligned}
$$

We can see that this isomorphism coincides with the homomorphism we have constructed, and the theorem follows.

REMARK. We may be able to weaken the assumption of the theorem. The theorem should hold only by assuming that $X_{0} \backslash Z \rightarrow Y_{0} \backslash W$ is smooth. Moreover, we may be able to see the theorem as a solution of a part of "Cauchy-Kovalevskaya type problem". Classically, this observation first appeared in Kashiwara's thesis [Ka], and the problem was interpreted in terms of the language of $\mathscr{D}$-modules. We expect that the analogous theorem also holds in our setting: if the morphism $f$ is "non-characteristic" to a coherent $F-\mathscr{D}_{\mathscr{K}, \mathrm{Q}}^{\dagger}$-module, then we get the isomorphism (3.10.1).

3.11 Corollary. Let $\mathscr{B}$ be a smooth formal scheme. Let $d$ be the dimension of $\mathscr{X}$, and $Z$ be a divisor of the special fiber of $\mathscr{X}$. Then we get a canonical isomorphism

$$
\left.\mathrm{D}_{\mathscr{X}, Z}\left(\mathcal{O}_{\mathscr{X}, \mathrm{Q}}\left({ }^{\dagger} Z\right)\right) \stackrel{\sim}{\rightarrow} \mathcal{O}_{\mathscr{X}, \mathrm{Q}}{ }^{\dagger} Z\right)(-d)
$$

where d denotes the dimension of $\mathscr{X}$.

Proof. Apply Theorem 3.10 in the case where $\mathscr{Y}=\operatorname{Spf}(R)$. 
Explicit calculation of Frobenius isomorphisms and Poincaré duality etc. 123

REMARK. The question to calculate $\mathrm{D}\left(\mathcal{O}_{\mathscr{C}, \mathrm{Q}}\right)$ was posed by Caro in [Ca5, 4.3.3], saying that "En effet, lorsque $X=P$, on retrouve l'isomorphisme canonique: $\mathrm{D}_{\mathcal{P}, \mathcal{T}}\left(\mathcal{O}_{\mathcal{P}}\left({ }^{\dagger} T\right)_{\mathrm{Q}}\right) \rightarrow \mathcal{O}_{\mathcal{P}}\left({ }^{\dagger} T\right)_{\mathrm{Q}}$. Je n'ai pas de contre-exemple mais la compatibilité à Frobenius de ce dernier isomorphisme me paraît inexacte."

3.12. Let $\mathscr{C}$ be a smooth formal scheme of dimension $d, Z$ be a divisor of the special fiber $X_{0}$ of $\mathscr{C}$. For an overconvergent $F$-isocrystal $M$ on $X_{0}$, we denote by $M^{\vee}$ the dual overconvergent $F$-isocrystal of $M$.

Corollary. Let sp: $\mathscr{C}_{K} \rightarrow \mathscr{6}$ be the specialization map, and let $M$ be an overconvergent $F$-isocrystal on $X_{0} \backslash Z$. Then,

$$
\left(\mathrm{D}_{\mathscr{C}, Z}\left(\operatorname{sp}_{*}(M)\right) \cong \operatorname{sp}_{*}\left(M^{\vee}\right)(-d)\right.
$$

Proof. Apply Corollary 3.11 to [Ca3, 2.3.37].

REMARK. This corollary completes the comparison of $L$-functions of isocrystals and arithmetic $\mathscr{D}$-modules [Ca2, 3.3.1]. Namely, we get

$$
L(Y, E, t)=L\left(\mathscr{H}, \operatorname{sp}_{*} E, q^{d_{X}} \cdot t\right)
$$

using the notation of loc. cit. However, in loc. cit. the definition of the Frobenius structure of the push-forward is modified in order to make the relative duality compatible with Frobenius (cf. [Ca2, 1.2.11]), and, a priori, the definition may not be the same as that of Berthelot. Still, we will show that this duality is compatible with Frobenius in the next section (cf. Corollary 4.17), and the Frobenius structure of the push-forward is in fact the same as that of Berthelot.

3.13. Now, we will compare the rigid cohomologies and the push-forwards of arithmetic $\mathscr{D}$-modules with Frobenius structure when varieties can be lifted to smooth formal schemes. If we do not consider Frobenius structure, they coincide up to shifts of degree, which is a result of Berthelot (cf. [BeI, 4.3.6.3]). If we consider Frobenius structure, we need a Tate twist. This twist naturally appears in the philosophy of six functors (cf. paragraph 5.9).

Let us fix the notation. We consider the situation 2.1.2. Let $\mathscr{C}$ be a smooth formal scheme and let $p: \mathscr{C} \rightarrow \mathscr{S}:=\operatorname{Spf}(R)$ be the structural morphism. Let $X_{0}$ be the special fiber of $\mathscr{C}$ as usual, $\mathscr{C}^{\prime}$ be a lifting of $X_{0}^{(s)}$, and $\mathscr{C}_{K}$ be the Raynaud generic fiber. We denote by sp: $\mathscr{C}_{K} \rightarrow \mathscr{C}$ the specialization map of topoi. Let $\mathscr{C}$ be an $F-\mathscr{D}_{\mathscr{K}, Q}^{\dagger}$-module. We define the 
"rigid cohomology" of $\mathscr{C} 6$ in the following way. Let $p_{K}: \mathscr{C}_{K} \rightarrow \mathrm{Spm}(\mathrm{K})$ be the structural morphism. We define

$$
H_{\text {rig }}^{i}\left(X_{0}, \mathscr{C O}\right):=R^{i} p_{K *}\left(\Omega_{\mathscr{C}_{K}}^{\bullet} \otimes_{\mathcal{O}_{K}} \mathrm{sp}^{*} \mathscr{C l}\right) .
$$

We define the Frobenius structure in the following way. There exists an isomorphism

$$
\varphi: \Omega_{\mathscr{X}_{K}^{\prime}}^{\bullet} \otimes_{\mathcal{O}_{X^{\prime}}} \mathscr{\mathscr { C } ^ { \sigma }} \stackrel{\sim}{\rightarrow} \Omega_{\mathscr{C}_{K}}^{\bullet} \otimes_{\mathcal{O}_{X}} F^{*} \mathscr{l ^ { \sigma }} \stackrel{\sim}{\rightarrow} \Omega_{\mathscr{X}_{K}}^{\bullet} \otimes_{\mathcal{O}_{X}} \mathscr{l l}
$$

in $D^{+}(\mathscr{C})$ where the first isomorphism follows from [Be2, 4.3.5] and we used the Frobenius structure of $\mathscr{A} b$ in the second isomorphism. Thus, we get an isomorphism

$$
H_{\text {rig }}^{i}\left(X_{0}, \mathscr{C l}\right) \stackrel{\sim}{\rightarrow} H_{\text {rig }}^{i}\left(X_{0}^{(s)}, \mathscr{L l}^{\sigma}\right) \underset{\varphi}{\stackrel{\sim}{\rightarrow}} H_{\text {rig }}^{i}\left(X_{0}, \mathscr{C l}\right),
$$

where the first isomorphism is the base change homomorphism. This is the induced Frobenius structure on the cohomology.

Suppose moreover that $\mathscr{C}$ is smooth proper and let $Z$ be a divisor of $X_{0}$. When $M$ is an overconvergent $F$-isocrystal on $X_{0} \backslash Z$ along $Z$, the rigid cohomology of $\operatorname{sp}_{*}(M)$ is isomorphic to the usual rigid cohomology of $M$.

3.14. Theorem. We preserve the notation. We suppose that $\mathscr{X}$ is purely of dimension d. Let 16 be a coherent $F-\mathscr{D}_{\mathscr{C}, \mathrm{Q}}^{\dagger}$-module. Then we get

$$
H^{i} p_{+} \mathscr{L} \cong H_{\text {rig }}^{i+d}\left(X_{0}, \mathscr{L} b\right)(d) .
$$

PRoOF. Let $\mathscr{C}^{\prime}$ be a coherent $\mathscr{D}_{\mathscr{Q}}^{\dagger}, \mathrm{Q}$-module, and consider the following diagram of complexes in $D^{b}(\mathscr{B})$.

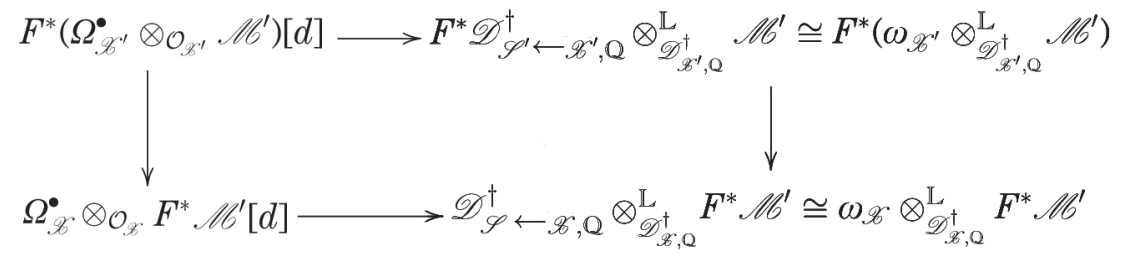

where the horizontal arrows are induced by [BeI, 4.2.1.1]. We need to see that this diagram is commutative up to multiplication by $q^{d}$. Indeed, this diagram is nothing but Lemma 3.6 by taking into account the proof of [Be2, 4.3.5].

Now to know the Frobenius actions on the cohomologies, apply $\mathscr{C}^{\prime}$ to be $\mathscr{C G}^{\sigma}$. We only need to take $R p_{*}$ to the four sheaves in the diagram with this $\mathscr{C l}^{\prime}$, and we get the theorem. 
3.15 Remark. (i) We can also compare in the relative situations. Namely, when we are given a smooth morphism of smooth formal schemes $\mathscr{X} \rightarrow \mathscr{Y}$, we are able to compare the relative rigid cohomology and the push-forward as arithmetic $\mathscr{D}$-module. Required methods are exactly the same, so we leave the precise formulation and calculation to the readers.

(ii) In [NH1, 6.2], Noot-Huyghe cited a calculation of BaldassarriBerthelot [BB]. However, the definition of Frobenius structures in [NH1] and $[\mathrm{BB}]$ are not the same, and we need a Tate twist here. Precisely, NootHuyghe used cohomological functors of the arithmetic $\mathscr{D}$-module theory to define the Frobenius structure. On the other hand Baldassarri-Berthelot used the relative rigid cohomologies to define the Frobenius structure on the Fourier transform. Thus we need to add the Tate twist $(N)$ on the right hand side of the isomorphism in [NH1, 6.2], namely

$$
\mathcal{F}_{\pi}\left(\mathcal{O}_{Y, \mathbf{Q}}^{\dagger}\right)[2-N] \cong \mathcal{H}_{X_{0}}^{\dagger N}\left(\mathcal{O}_{Y^{\vee}, \mathbf{Q}}^{\dagger}\right)(N)
$$

using the notation of loc. cit.

(iii) D. Caro pointed out to the author in personal communications that we need a suitable Tate twist in [Ca4, Proposition 2.3.12]. He also pointed out that some modifications might be needed in loc. cit. Theorem 3.3.4, in whose proof he used the proposition.

\section{Complementary results}

In this section, we will prove three complementary results; 1) commutation of the dual functor and the tensor product, 2) the Künneth formula, and 3) the compatibility of the relative duality isomorphism by Virrion with Frobenius. The first commutation result is another application of Theorem 3.14, and the proofs of 2) and 3) are independent from the other part of this paper. Although the Künneth formula for arithmetic $\mathscr{D}$-modules seems to be well-known to experts, we could not find any appropriate reference. We think that this would be a good occasion to include the proof. The compatibility of relative duality is needed to establish the Poincaré duality.

\section{Commutation of the dual functor and tensor product}

4.1. We consider the situation 2.1.1. Let $X$ be a smooth scheme over $\operatorname{Spec}\left(R_{i}\right)$ for some $i$, and let $Z$ be a divisor. We put $\widehat{\mathcal{B}}_{\mathscr{X}, Q}^{(m)}:=\widehat{\mathcal{B}}_{\mathscr{C}, Q}^{(m)}(Z)$, and $\widetilde{\mathscr{D}}_{\mathscr{X}, \mathrm{Q}}^{(m)}:=\widehat{\mathcal{B}}_{\mathscr{X}, \mathrm{Q}}^{(m)} \widehat{\otimes}_{\mathcal{O}_{\mathscr{X}}} \widehat{\mathscr{D}}_{\mathscr{X}}^{(m)}$. We denote the dual functor with respect to $\widetilde{\mathscr{D}}_{\mathscr{X}, \mathrm{Q}}^{(m)}$ by $\mathrm{D}$. First, we get the following lemma. 
LEMMA. Let $\mathscr{l}$ be a complex in $D_{\text {perf }}^{b}\left(\widetilde{\mathscr{D}}_{\mathscr{X}, \mathrm{Q}}^{(m)}\right)$, and $\mathscr{N}$ be a coherent $\widetilde{\mathscr{D}}_{\mathscr{X}, \mathrm{Q}}^{(m)}$-module which is also coherent as a $\widehat{\mathcal{B}}_{\mathscr{X}, \mathrm{Q}}^{(m)}$-module. We denote the dimension of $\mathscr{B}$ by $d$. Then, we have the following isomorphism

$$
\kappa: \mathrm{D}(\mathscr{C l}) \otimes_{\widehat{\mathcal{B}}_{X ; Q}^{(m)}}^{\mathrm{L}} \mathscr{N} \cong R \mathcal{H o m}_{\widetilde{\mathscr{D}}_{\mathscr{X}, \mathrm{Q}}^{(m)}}\left(\mathscr{L l}, \widetilde{\mathscr{D}}_{\mathscr{X}, \mathrm{Q}}^{(m)} \otimes_{\widehat{\mathcal{B}}_{\mathscr{X}, \mathrm{Q}}^{(m)}}^{\mathrm{L}} \mathscr{N}\right) \otimes \omega_{\mathscr{X}}^{-1}[d]
$$

of complexes in $D^{b}\left(\widetilde{\mathscr{D}}_{\mathscr{X}, \mathrm{Q}}^{(m)}\right)$. Here, the right module structure of $\widetilde{\mathscr{D}}_{\mathscr{C}, \mathrm{Q}}^{(m)} \otimes_{\widehat{\mathcal{B}}_{\mathscr{X}, Q}^{(m)}}^{\mathrm{L}} \mathscr{N}$ is defined by that of $\widetilde{\mathscr{D}}_{\mathscr{C}, Q}^{(m)}$, and the left structure by [Be2, 1.1.7]. Moreover, this isomorphism is compatible with Frobenius.

Proof. By [Be1, 4.4.2], $\mathscr{N}$ is a locally projective $\widehat{\mathcal{B}}_{\mathscr{K}, Q}^{(m)}$-module, and we do not need to take the derived tensor products. Let $\mathscr{L}^{\prime}$ be a right $\widetilde{\mathscr{D}}_{\mathscr{O}, Q^{-}}^{(m)}$ module. Then $\mathscr{C}^{\prime} \otimes_{\widehat{\mathcal{B}}_{\mathscr{C}, Q}^{(m)}} \mathscr{N}$ possesses a right $\widetilde{\mathscr{D}}_{\mathscr{K}, \mathrm{Q}}^{(m)}$-module structure. Indeed let $\mathscr{\mathscr { C }}$ be an affine open formal subscheme of $\mathscr{b}$. Let $M^{\prime}:=\Gamma\left(\mathscr{C}, \mathscr{C l}^{\prime}\right)$, $N:=\Gamma(\mathscr{C}, \mathscr{N}), B:=\Gamma\left(\mathscr{C}, \widehat{\mathcal{B}}_{\mathscr{X}, Q}^{(m)}\right)$, and $D:=\Gamma\left(\mathscr{C}, \widetilde{\mathscr{D}}_{\mathscr{K}, Q}^{(m)}\right)$. Then it suffices to define a right $D$-module structure on $M^{\prime} \otimes_{B} N$. For $a \otimes b \in M^{\prime} \otimes_{B} N$ and $P \in D$, it suffices to define $(a \otimes b) \cdot P$. Take $S \subset M^{\prime}$ to be the finite $\Gamma\left(\mathscr{C}, \widetilde{\mathscr{D}}_{\mathscr{C}, \mathrm{Q}}^{(m)}\right)$-submodule generated by $a$. Then $S \otimes N$ is naturally a $D$ module considering [Be1, 4.4.7] and [Be2, 1.1.7]. This defines $(a \otimes b) \cdot P$.

Now back to the proof, by using [Vi, I, 1.2.2], we get an isomorphism $\kappa$ of complexes in $D^{b}\left(\widehat{\mathcal{B}}_{\mathscr{C}, Q}^{(m)}\right)$. It suffices to show that this isomorphism is an

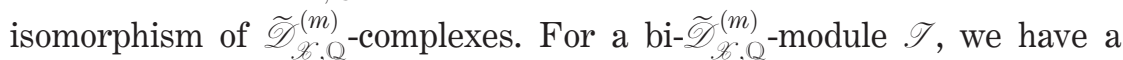
canonical homomorphism

$$
\mathcal{H o m}_{\widetilde{\mathscr{D}}_{\mathscr{X}, Q}^{(m)}}(\mathscr{L l}, \mathscr{T}) \otimes_{\widehat{\mathcal{B}}_{x ; Q}^{(m)}} \mathscr{N} \rightarrow \mathcal{H o m}_{\widetilde{\mathscr{D}}_{x, Q}^{(m)}}\left(\mathscr{L l}, \mathscr{T} \otimes_{\widehat{\mathcal{B}}_{x ; Q}^{(m)}} \mathscr{N}\right) .
$$

A priori, this is a homomorphism of $\widehat{\mathcal{B}}_{\mathscr{C}, Q}^{(m)}$-modules. By the argument above, both sides of the homomorphism possess the right $\widetilde{\mathscr{D}}_{\mathscr{K}, \mathrm{Q}}^{(m)}$-module structures. To finish the proof, is suffices to see that the homomorphism is $\widetilde{\mathscr{D}}_{\mathscr{X}, Q^{-}}^{(m)}$ linear. The verification is straightforward.

4.2. Now, consider the situation 2.1.2. Let $\mathscr{B}$ be a smooth formal scheme over $\operatorname{Spf}(R)$, and $Z$ be a divisor of the special fiber. Let $\mathscr{N}^{\prime}$ be a coherent $F$ $\mathscr{D}_{\mathscr{X}, \mathrm{Q}}^{\dagger}\left({ }^{\dagger} Z\right)$-module which is also coherent as an $\mathcal{O}_{\mathscr{C}, \mathrm{Q}}\left(^{\dagger} Z\right)$-module. By abuse of language, we say that $\mathscr{N}^{\prime}$ is a convergent $F$-isocrystal overconvergent along $Z$ (cf. Notation 0.5 ). We put $N^{\prime}:=\operatorname{sp}^{*}\left(N^{\prime}\right)$, which is an overconvergent $F$ -

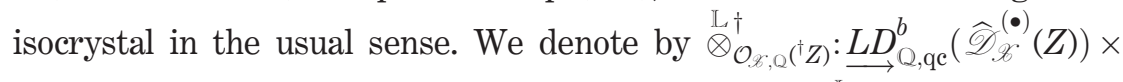

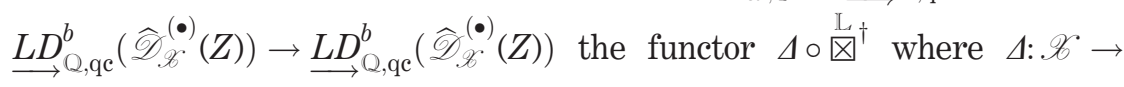


Explicit calculation of Frobenius isomorphisms and Poincaré duality etc. 127

$\mathscr{B} \times \mathscr{f}$ (cf. [BeI, 3.4.7]). Thanks to Corollary 3.12, we get the following isomorphisms of bimodules. We omit subscripts Q and denote by $(Z)$ instead of $\left({ }^{\dagger} Z\right)$ in the next equality to save the space.

(4.2.1) $\operatorname{RHom}_{\mathcal{O}_{\mathscr{X}}(Z)}\left(\mathbb{D}_{\mathscr{X}, Z}\left(\mathscr{N}^{\prime}\right), \mathscr{D}_{\mathscr{X}}^{\dagger}(Z)\right)$

$\cong \mathscr{D}_{\mathscr{X}}^{\dagger}(Z) \otimes_{\mathcal{O}_{\mathscr{X}}(Z)} R \mathcal{H o m}_{\mathcal{O}_{\mathscr{X}}(Z)}\left(\operatorname{sp}_{*}\left(N^{\prime \vee}\right)(-d), \mathcal{O}_{\mathscr{X}}(Z)\right) \cong \mathscr{D}_{\mathscr{X}}^{\dagger}(Z){\stackrel{\mathrm{L}}{\dagger} \otimes_{\mathcal{O}}^{\dagger}(Z)}^{N^{\prime}}(d)$

Here the right module structure of the first module is defined by [Be2, 1.1.7] using the right module structure of $\mathscr{D}_{\mathscr{X}, Q}^{\dagger}\left({ }^{\dagger} Z\right)$, and the left structure by using that of $\mathscr{D}_{\mathscr{C}, Q}^{\dagger}\left({ }^{\dagger} Z\right)$. This is compatible with Frobenius, which means that the following diagram of canonical isomorphisms is commutative. We again omit $Q$ and denote by $(Z)$ in the following.

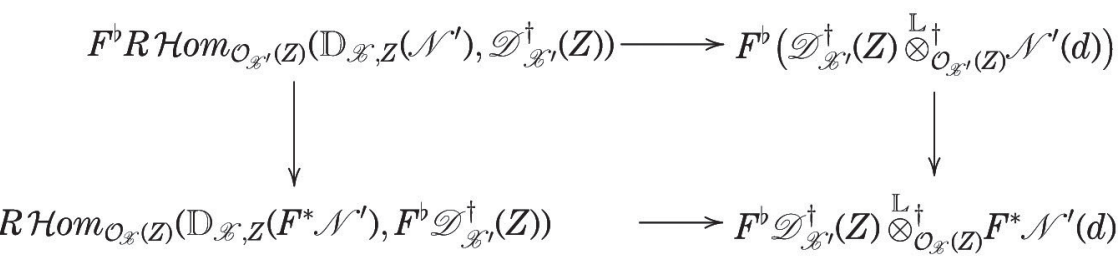

Here the homomorphisms are the canonical ones except for the right vertical homomorphism, which is $q^{-d}$ times the canonical homomorphism.

Let $\mathscr{C}$ be an object in $F-D_{\text {perf }}^{b}\left(\mathscr{D}_{\mathscr{X}, \mathrm{Q}}^{\dagger}\left({ }^{\dagger} Z\right)\right)$. We get the following isomorphisms compatible with Frobenius structures:

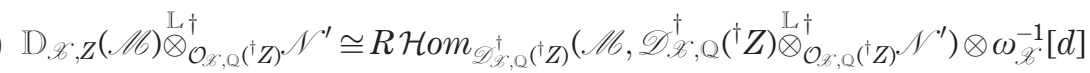

$\cong R \mathcal{H o m}_{\left.\mathscr{D}_{\mathscr{X}, Q}^{\dagger}{ }^{\dagger} Z\right)}\left(\mathscr{l}, R \mathcal{H o m}_{\left.\mathcal{O}_{\mathscr{X}, Q}{ }^{\dagger} Z\right)}\left(\mathrm{D}_{\mathscr{X}, Z}\left(\mathscr{N}^{\prime}\right), \mathscr{D}_{\mathscr{X}, \mathrm{Q}}^{\dagger}\left({ }^{\dagger} Z\right)\right)\right) \otimes \omega_{\mathscr{K}}^{-1}(-d)[d]$

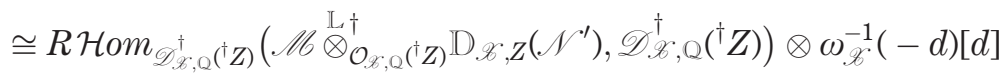

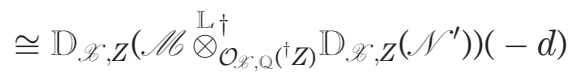

where the first isomorphism by Lemma 4.1, the second by (4.2.1), and the third by using [Ca3, 2.1.34]. Now, we get the following proposition.

4.3 Proposition. Let $\mathscr{B}$ be a smooth formal scheme over $\operatorname{Spf}(R)$, and $Z$ be a divisor of its special fiber. Let $\mathscr{L}$ be a coherent $\left.F-\mathscr{D}_{\mathscr{R}, Q}^{\dagger}{ }^{\dagger} Z\right)$-module, and $\mathscr{N}$ be an overconvergent $F$-isocrystal along $Z$. Then we get

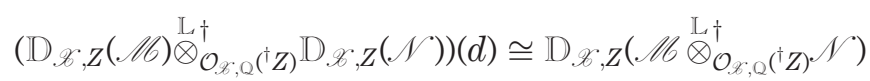

which is compatible with Frobenius structures. 
Proof. Take $\mathscr{N}^{\prime}:=\mathbb{D}_{\mathscr{K}, Z}(\mathscr{N})$, and the isomorphism (4.2.2) induces the isomorphism we are looking for.

REMARK. We are not able to expect the isomorphism (4.3.1) in general. For example, consider the closed immersion $i:\{0\} \hookrightarrow \widehat{A}_{R}$. Then taking $\mathscr{C} b=\mathscr{N}=i_{+} K$, we do not have such an isomorphism.

\section{The Künneth formula}

4.4. Now, we will show the Künneth formula. The ideas used to show the

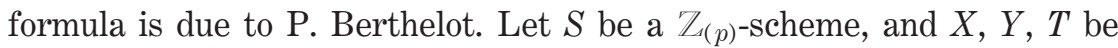
smooth formal schemes over $S$. Consider the following commutative diagram:

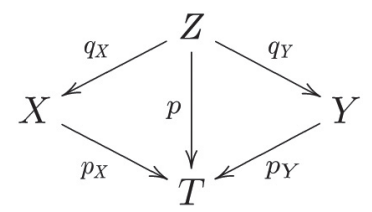

where $Z=X \times_{T} Y$. Suppose that $p_{X}$ and $p_{Y}$ are smooth.

4.5. Let $\mathcal{B}_{T}$, (resp. $\mathcal{B}_{X}, \mathcal{B}_{Y}$ ) be a commutative $\mathcal{O}_{T}$-algebra (resp. $q_{X}^{*} \mathcal{B}_{T}$-algebra, $q_{Y}^{*} \mathcal{B}_{Y}$-algebra) endowed with an action of $\mathscr{D}_{T}^{(m)}\left(\right.$ resp. $\left.\mathscr{D}_{X}^{(m)}, \mathscr{D}_{Y}^{(m)}\right)$ compatible with that of $\mathcal{O}_{T}\left(\operatorname{resp} . \mathcal{O}_{X}, \mathcal{O}_{Y}\right)$. For a $\mathcal{B}_{X}$-module $\mathscr{F}$ and a $\mathcal{B}_{Y}$ module $\mathscr{G}$, we put $\mathscr{F} \otimes_{\mathcal{B}_{T}} \mathscr{G}:=q_{X}^{*} \mathscr{F} \otimes_{p^{*} \mathcal{B}_{T}} q_{Y}^{*} \mathscr{G}$. Let $\mathcal{B}_{Z}:=\mathcal{B}_{X} \otimes_{\mathcal{B}_{T}} \mathcal{B}_{Y}$. We put $\widetilde{\mathscr{D}}_{*}^{(m)}:=\mathcal{B}_{*} \otimes \mathscr{D}_{*}^{(m)}$ where $* \in\{X, Y, Z\}$. We note that $p^{*} \widetilde{\mathscr{D}}_{T}^{(m)}$ is a sub$\mathcal{O}_{Z}$-algebra of $\widetilde{\mathscr{D}}_{Z}^{(m)}$. We get the following lemma.

LEMma. We preserve the notation.

(i) There exists the canonical isomorphism

$$
\widetilde{\mathscr{D}}_{Z}^{(m)} \cong q_{X}^{*} \widetilde{\mathscr{D}}_{X}^{(m)} \otimes_{p^{*} \widetilde{\mathscr{V}}_{T}^{(m)}} q_{Y}^{*} \widetilde{\mathscr{D}}_{Y}^{(m)} .
$$

(ii) There exists the canonical isomorphism

$$
\widetilde{\mathscr{D}}_{T \leftarrow Z}^{(m)} \cong q_{X}^{*} \widetilde{\mathscr{D}}_{T \leftarrow X}^{(m)} \otimes_{p^{*} \widetilde{\mathscr{D}}_{T}^{(m)}} q_{Y}^{*} \widetilde{\mathscr{D}}_{T \leftarrow Y}^{(m)} .
$$

Proof. The natural homomorphisms $q_{X}^{*} \widetilde{\mathscr{D}}_{X}^{(m)} \rightarrow \widetilde{\mathscr{D}}_{Z}^{(m)}$ and $q_{Y}^{*} \widetilde{\mathscr{D}}_{Y}^{(m)} \rightarrow$ $\widetilde{\mathscr{D}}_{Z}^{(m)}$ induces the homomorphism $q_{X}^{*} \widetilde{\mathscr{D}}_{X}^{(m)} \otimes_{p^{*} \widetilde{\mathscr{D}}_{T}^{(m)}} q_{Y}^{*} \widetilde{\mathscr{D}}_{Y}^{(m)} \rightarrow \widetilde{\mathscr{D}}_{Z}^{(m)}$. To see that this is an isomorphism, we may assume that $T$ possesses a system of local 
Explicit calculation of Frobenius isomorphisms and Poincaré duality etc. 129

coordinate and $X$ and $Y$ possesses a system of local coordinate over $T$. Then proof is straightforward, and we leave the reader for the detail. Let us see (ii). We know the following isomorphisms

$$
\omega_{Z / T} \cong q_{X}^{*} \omega_{X / T} \otimes_{\mathcal{O}_{Z}} q_{Y}^{*} \omega_{Y / T}
$$

We get

$$
\begin{aligned}
\widetilde{\mathscr{D}}_{T \leftarrow Z}^{(m)} & \cong p^{*} \widetilde{\mathscr{D}}_{T}^{(m)} \otimes_{\mathcal{O}_{Z}} \omega_{Z / T} \\
& \cong\left(q_{X}^{*} p_{X}^{*} \widetilde{\mathscr{D}}_{T}^{(m)} \otimes_{p^{*}} \widetilde{\mathscr{D}}_{T}^{(m)} q_{Y}^{*} p_{Y}^{*} \widetilde{\mathscr{D}}_{T}^{(m)}\right) \otimes_{\mathcal{O}_{Z}}\left(q_{X}^{*} \omega_{X / T} \otimes q_{Y}^{*} \omega_{Y / T}\right) \\
& \cong q_{X}^{*} \widetilde{\mathscr{D}}_{T \leftarrow X}^{(m)} \otimes_{p^{*} \widetilde{\mathscr{D}}_{T}^{(m)}} q_{Y}^{*} \widetilde{\mathscr{D}}_{T \leftarrow Y}^{(m)} .
\end{aligned}
$$

4.6 Lemma. Let $\mathscr{l}$ be a left flat $\widetilde{\mathscr{D}}_{X}^{(m)}$-module, and $\mathscr{N}$ be a left flat $\widetilde{\mathscr{D}}_{Y}^{(m)}$ module. Then we get that $\mathscr{C l}_{\mathcal{B}_{T}}$ N is a flat left $\widetilde{\mathscr{D}}_{Z}^{(m)}$-module.

Proof. In the case where $T=\operatorname{Spf}(R)$, the verification is left to the readers. To see the lemma, since the verification is local, we may assume that $T$ is affine. Let $i: Z=X \times_{T} Y \hookrightarrow W:=X \times Y$ be the canonical inclusion. Since $T$ is separated, this is a closed immersion. By the $T=\operatorname{Spf}(R)$ case, we get that $\mathscr{C} \bigotimes_{S} \mathscr{N}$ is a flat $\widetilde{\mathscr{D}}_{W}^{(m)}$-module. We put $\widetilde{\mathscr{D}}_{Z \rightarrow W}^{(m)}:=i^{*} \widetilde{\mathscr{D}}_{W}^{(m)}$, which is a $\left(\widetilde{\mathscr{D}}_{Z}^{(m)}, i^{-1} \widetilde{\mathscr{D}}_{W}^{(m)}\right)$-module as usual. Let $\mathscr{F}$ be a right $\widetilde{\mathscr{D}}_{Z}^{(m)}$-module. Then we get

$$
\mathscr{F} \otimes_{\widetilde{\mathscr{D}}_{Z}^{(m)}}\left(\mathscr{L} \bigotimes_{\mathcal{B}_{T}} \mathscr{N}\right) \cong\left(\mathscr{F} \otimes_{\widetilde{\mathscr{V}}_{Z}^{(m)}} \widetilde{\mathscr{D}}_{Z \rightarrow W}^{(m)}\right) \otimes_{i^{-1} \widetilde{\mathscr{D}}_{W}^{(m)}} i^{-1}\left(\mathscr{L} \bigotimes_{S} \mathscr{N}\right) .
$$

Since $\widetilde{\mathscr{D}}_{Z \rightarrow W}^{(m)}$ is flat over $\widetilde{\mathscr{D}}_{Z}^{(m)}$ and $\mathscr{C} \bigotimes_{S} \mathscr{N}$ is flat over $\widetilde{\mathscr{D}}_{W}^{(m)}$, we get the lemma.

Using this preparation, we get the following Künneth formula.

4.7 Proposition. We preserve the notation. Let $\mathscr{l l}$ (resp. $\mathscr{N}$ ) be a complex in $D_{\mathrm{qc}}^{-}\left(\widetilde{\mathscr{D}}_{X}^{(m)}\right)$ (resp. $D_{\mathrm{qc}}^{-}\left(\widetilde{\mathscr{D}}_{Y}^{(m)}\right)$ ). Then we get a canonical isomorphism in $D_{\mathrm{qc}}^{-}\left(\widetilde{\mathscr{D}}_{T}^{(m)}\right)$

$$
p_{+}\left(\mathscr{C} \otimes_{\mathcal{B}_{T}}^{\mathrm{L}} \mathscr{N}\right) \cong p_{X+}(\mathscr{C l}) \otimes_{\mathcal{B}_{T}}^{\mathrm{L}} p_{Y+}(\mathscr{N}) .
$$

Proof. Let $\mathscr{F}$ (resp. $\mathscr{G})$ be a quasi-coherent $\widetilde{\mathscr{D}}_{X}^{(m)}$-module (resp. $\widetilde{\mathscr{D}}_{Y}^{(m)}$ module). Then by Lemma 4.5, we get a canonical isomorphism of $p^{-1} \widetilde{\mathscr{D}}_{T}^{(m)}$ modules

$$
\widetilde{\mathscr{D}}_{T \leftarrow Z}^{(m)} \otimes_{\widetilde{\mathscr{D}}_{Z}^{(m)}}\left(\mathscr{F} \otimes_{\mathcal{B}_{T}} \mathscr{G}\right) \cong\left(\widetilde{\mathscr{D}}_{T \leftarrow X}^{(m)} \otimes_{\widetilde{\mathscr{D}}_{X}^{(m)}} \mathscr{F}\right) \bigotimes_{\mathcal{B}_{T}}\left(\widetilde{\mathscr{D}}_{T \leftarrow Y}^{(m)} \otimes_{\widetilde{\mathscr{D}}_{Y}^{(m)}} \mathscr{G}\right)
$$


Let $\mathscr{L} \cdot$ be a flat resolution of $\mathscr{F}$ as a $\widetilde{\mathscr{D}}_{X}^{(m)}$-module, and $\mathscr{C} \cdot$ be a flat resolution of $\mathscr{G}$ as a $\widetilde{\mathscr{D}}_{Y}^{(m)}$-module. Then we get that $\mathscr{L}_{i} \bigotimes_{\mathcal{B}_{T}} \mathscr{C}_{j}$ is a flat $\widetilde{\mathscr{D}}_{Z}^{(m)}$ module for any $i$ and $j$ by Lemma 4.6. Thus we get

$$
\begin{aligned}
& \widetilde{\mathscr{D}}_{T \leftarrow Z}^{(m)} \otimes_{\widetilde{\mathscr{D}}_{Z}^{(m)}}^{\mathrm{L}}\left(\mathscr{F} \otimes_{\mathcal{B}_{T}}^{\mathrm{L}} \mathscr{G}\right) \cong \widetilde{\mathscr{D}}_{T \leftarrow Z}^{(m)} \otimes_{\widetilde{D}_{Z}^{(m)}}\left(\mathscr{L} \cdot \otimes_{\mathcal{B}_{T}}^{\mathrm{L}} \mathscr{C l}_{\bullet}\right) \\
& \cong\left(\widetilde{\mathscr{D}}_{T \leftarrow X}^{(m)} \otimes_{\widetilde{\mathscr{D}}_{X}^{(m)}} \mathscr{L}_{\bullet}\right) \bigotimes_{\mathcal{B}_{T}}\left(\widetilde{\mathscr{D}}_{T \leftarrow Y}^{(m)} \otimes_{\widetilde{\mathscr{D}}_{Y}^{(m)}} \mathscr{C l}_{\bullet}\right) \\
& \cong\left(\widetilde{\mathscr{D}}_{T \leftarrow X}^{(m)} \otimes_{\widetilde{\mathscr{D}}_{X}^{(m)}}^{\mathrm{L}} \mathscr{F}\right) \bigotimes_{\mathcal{B}_{T}}^{\mathrm{L}}\left(\widetilde{\mathscr{D}}_{T \leftarrow Y}^{(m)} \otimes_{\widetilde{\mathscr{D}}_{Y}^{(m)}}^{\mathrm{L}} \mathscr{Y}\right) .
\end{aligned}
$$

By using the Künneth formula for quasi-coherent sheaves, we get the proposition.

4.8. Now, let us consider Frobenius. Suppose $T$ is endowed with a quasicoherent $m$-PD-ideal $(\mathfrak{a}, \mathfrak{b}, \alpha)$, and $p \in \mathfrak{a}$. With this hypothesis, we are able to consider Frobenius pull-back even if there are no liftings of relative Frobenius morphisms.

LEMma. Suppose that $X_{0}$ and $Y_{0}$ be the reductions of $X$ and $Y$ respectively, and $X^{\prime}, Y^{\prime}$ be liftings of $X_{0}^{(s)}, Y_{0}^{(s)}$. We take $Z^{\prime}:=X^{\prime} \times_{T} Y^{\prime}$. Then the isomorphism (4.7.1) is compatible with Frobenius isomorphisms. Moreover, if a is m-PD-nilpotent, it is compatible with Frobenius isomorphism even if there are no liftings.

Proof. The verification uses only standard arguments, so we leave the details to the readers.

4.9. Finally, by taking inverse limit and inductive limit, we get the following Künneth formula for $\mathscr{D}^{\dagger}$-modules.

Proposition. Consider the following diagram

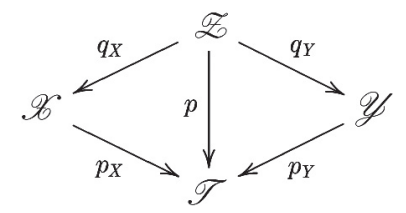

where $\mathscr{T}$ is a smooth formal scheme, $p_{X}$ and $p_{Y}$ are smooth, and $\mathscr{Z}:=$ $\mathscr{B} \times \mathscr{T} \mathscr{Y}$. Let $D$ be a divisor of the special fiber of $\mathscr{T}, D_{X}\left(r e s p . D_{Y}\right)$ be a divisor of the special fiber of $\mathscr{B}$ (resp. $\mathscr{Y}$ ) such that $D_{X} \supset p_{X}^{-1}(D)$ (resp. 
Explicit calculation of Frobenius isomorphisms and Poincaré duality etc. 131

$\left.D_{Y} \supset p_{Y}^{-1}(D)\right)$. Let $\mathscr{C l}($ resp. $\mathscr{N})$ be a complex in $\left.\underset{L D_{\mathrm{Q}, \mathrm{qc}}^{b}}{\left(\widehat{\mathscr{D}}_{\mathscr{O}}^{(\bullet)}\right.}\left(D_{X}\right)\right)$ (resp. $\left.\stackrel{L D_{Q, q c}^{b}}{\longrightarrow}\left(\widehat{\mathscr{D}}_{\mathscr{Y}}^{(\bullet)}\left(D_{Y}\right)\right)\right)$. Then we get the canonical isomorphism

$$
p_{+}\left(\mathscr{C} \mathbb{Q}_{\mathcal{O}_{\mathscr{T}}\left({ }^{\dagger} D\right)}^{\dagger} \mathscr{N}\right) \cong p_{X+}(\mathscr{C}){\stackrel{\mathrm{L}}{\mathrm{L}^{\dagger}}}_{\mathcal{O}_{\mathscr{T}}\left({ }^{\dagger} D\right)} p_{Y+}(\mathscr{N})
$$

in $\underset{L D_{\mathrm{Q}, \mathrm{qc}}^{b}}{b}\left(\widehat{\mathscr{D}}_{\mathscr{T}}^{(\bullet)}(D)\right)$. This isomorphism is compatible with the Frobenius isomorphisms.

4.10 REMARK. To get the proposition directly, we can also proceed as follows. Consider the following cartesian diagram.

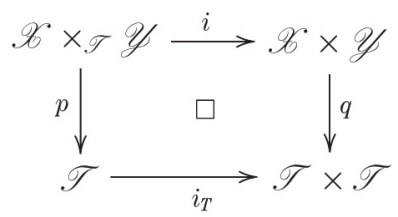

We see easily that $q_{+}\left(\mathscr{C} \otimes^{\mathrm{L}} \mathscr{N}\right) \cong q_{+}(\mathscr{C l}) \bigotimes_{\mathcal{O}_{\mathscr{T} \times \mathscr{T}}}^{\mathrm{L}} q_{+}(\mathscr{N})$. Let $\mathscr{S}:=\operatorname{Spf}(R)$. By using this and [Ca1, 2.1.9], we get

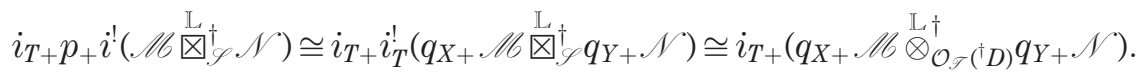

Taking $H^{0} i_{T}^{!}$, we get what we want.

\section{Compatibility of Frobenius pull-backs with relative duality}

4.11. We will show that the relative duality homomorphism by Virrion is compatible with Frobenius pull-back.

First, let us fix the situation. We consider the situation 2.1.1. Let $f: \mathscr{C} \rightarrow \mathscr{H}$ be a proper morphism of smooth formal schemes, and $W$ be a divisor of the special fiber of $\mathscr{H}$ such that $Z:=f^{-1}(W)$ is a divisor. Under this situation, Virrion [Vi2] defined the trace homomorphism

$$
\operatorname{Tr}_{+, f}: f_{+} \omega_{\mathscr{X}, \mathrm{Q}}\left[d_{X}\right] \rightarrow \omega_{\mathscr{Y}, \mathrm{Q}}\left[d_{Y}\right]
$$

where $d_{X}$ and $d_{Y}$ denotes the dimension of $\mathscr{B}$ and $\mathscr{Y}$. Using the trace map, for an object $\mathscr{E}$ in $D_{\text {perf }}^{b}\left(\mathscr{D}_{\mathscr{C}, Q}^{\dagger}\left({ }^{\dagger} Z\right)\right)$, she also constructed the relative duality isomorphism

$$
\chi: \mathbb{D}_{\mathscr{Y}, W} f_{+}(\mathscr{E}) \stackrel{\sim}{\rightarrow} f_{+} \mathrm{D}_{\mathscr{K}, Z}(\mathscr{E})
$$

in $D_{\text {perf }}^{b}\left(\mathscr{D}_{\mathscr{Y}, \mathrm{Q}}^{\dagger}\left({ }^{\dagger} W\right)\right.$ ) (see also [Ca2, 1.2.7]). Now, we assume that there exist liftings $\mathscr{C}^{\prime}$ and $\mathscr{H}^{\prime}$ of $X_{0}^{(s)}$ and $Y_{0}^{(s)}$ where $X_{0}$ and $Y_{0}$ are special fibers 
as usual. We also assume that there exists a lifting $f^{\prime}: \mathscr{C}^{\prime} \rightarrow \mathscr{Y}^{\prime}$ of the morphism $X_{0}^{(s)} \rightarrow Y_{0}^{(s)}$ induced by $f$. These assumptions automatically hold when we consider the situation 2.1.2.

4.12. Before stating the theorem, we will prepare a commutative diagram, which is needed in the proof of the compatibility. We freely use the notation of [Ha]. Let $X, X^{\prime}, Y, Y^{\prime}$ be locally noetherian schemes. Suppose we are given the following commutative diagram.

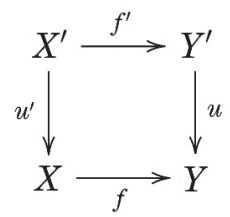

We assume that all the morphisms are proper, $u$ is finite flat, and all the schemes admit dualizing complexes (cf. [Ha, V, § 2]). By the transitivity of trace map [Ha, VI, 4.2(a) TRA1], we get the following commutative diagram.

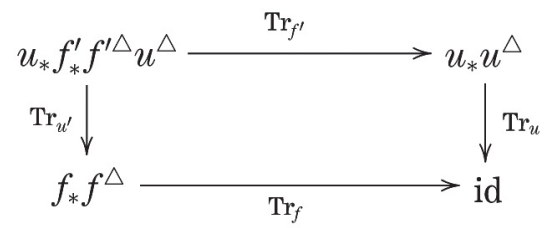

Here, $\operatorname{Tr}_{u^{\prime}}$ denotes the composition $u_{*} f_{*}^{\prime} f^{\prime \triangle} u^{\triangle} \cong f_{*} u_{*}^{\prime} u^{\prime} \triangle f^{\triangle} \stackrel{f_{*} \circ \operatorname{Tr}_{u^{\prime} \circ f^{\Delta}}}{\longrightarrow}$ $f_{*} f^{\triangle}$. We remind that this diagram consists of homomorphisms in the category of complexes by [Ha, VII, 2.1]. Let $u^{\circ}$ denotes the functor $u^{\prime}$ in [Ha, VI, 4.1] to avoid confusions with the morphism $u^{\prime}$. We note that $u^{\bullet} \cong u^{b} \cong u^{!}$ in the derived category since $u$ is finite and flat. We have the canonical homomorphism id $\rightarrow u^{\bullet} u_{*}$. Let $c: f_{*}^{\prime} f^{\prime \triangle} u^{\triangle} \rightarrow u^{\bullet} u_{*} f_{*}^{\prime} f^{\prime \triangle} u^{\triangle} \rightarrow u^{\bullet} f_{*} f^{\triangle}$ where the second morphism is that induced by the left vertical morphism $\operatorname{Tr}_{u^{\prime}}$ in the diagram above. Let $c^{\prime}: u^{\triangle} \rightarrow u^{\bullet} u_{*} u^{\triangle} \rightarrow u^{\bullet}$ where the second homomorphism is the trace map. Taking $u^{\bullet}$ to the above diagram, we get the following commutative diagram of complexes.

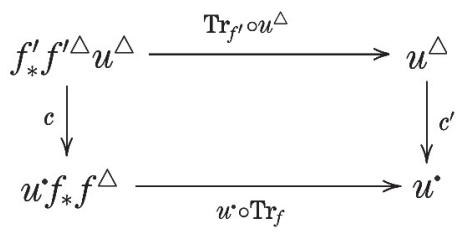


Explicit calculation of Frobenius isomorphisms and Poincaré duality etc. 133

The morphism id $\rightarrow u^{\bullet} u_{*} \cong u^{b} u_{*}$ is nothing but the adjunction homomorphism, and $c^{\prime}$ is the identity in the derived category.

4.13 Proposition. We preserve the assumptions and notation of paragraph 4.11. We moreover assume that $f$ can be factorized into morphisms $\mathscr{O} \stackrel{g}{\rightarrow} \mathscr{C}^{\prime} \stackrel{f^{\prime}}{\rightarrow} \mathscr{H}$ where $g$ is a closed immersion and $f^{\prime}$ is proper smooth. Then the relative duality isomorphism (4.11.2) is compatible with Frobenius pull-back.

Proof. Recall that $d_{X}$ (resp. $d_{Y}$ ) was the dimension of $\mathscr{C}$ (resp. $\mathscr{Y})$. We let $d$ to be the relative dimension, namely $d:=d_{X}-d_{Y}$. We denote by $f_{+}^{(m)}$ the push-forward of level $m$. We will use the pushforward for right modules as in [Vi2, III]: recall that $f_{+}^{(m)}(\mathscr{C l}):=$ $R f_{*}\left(\mathscr{C} \otimes \widehat{\mathscr{D}}_{\mathscr{R}, \mathrm{Q}}^{\mathrm{L}} \widehat{\mathscr{D}}_{\mathscr{C} \rightarrow \mathscr{Y}, \mathrm{Q}}^{(m)}\right)$ for a coherent right $\widehat{\mathscr{D}}_{\mathscr{O}, \mathrm{Q}}^{(m)}$-module $\mathscr{C}$. It suffices to show that the trace homomorphism (4.11.1) is compatible with Frobenius pull-backs by [Ca7, 1.5]. By the result of Caro [Ca7, 2.2.7], we know the compatibility in the case where $f$ is a closed immersion. Thus, we are reduced to showing the case where $f$ is smooth using the transitivity of the trace map [Vi2, III, 5.5]. In the following, we assume $f$ to be smooth.

Let $i$ be a non-negative integer, and $X$ and $Y$ be the reductions of $\mathscr{C}$ and $\mathscr{H}$ over $R_{i}$. It suffices to show the compatibility for these $X$ and $Y$, namely we need to prove the commutativity of the following diagram.

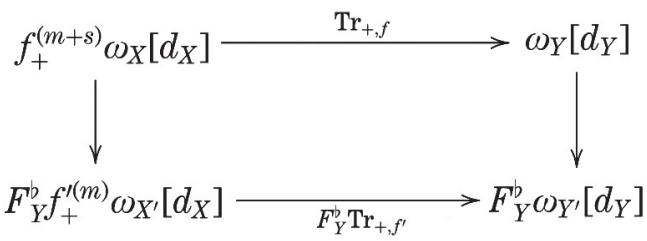

Since $f$ is smooth and the relative dimension is $d$, we get that for any point $y$ in $\mathscr{H}$, the dimension of $f^{-1}(y)$ is equal to $d$. Since $f$ is proper, $R^{i} f_{*}(\mathscr{F})=0$ for any quasi-coherent sheaf $\mathscr{F}$ and $i>d$ (cf. for example [Kl, I Lemma (3)]). Thus, by the definition of $f_{+}$, the canonical homomorphism

$$
R^{d} f_{*} \omega_{X} \rightarrow H^{d} f_{+}^{(m)} \omega_{X}
$$

is surjective, and $H^{i} f_{+}^{(m)} \omega_{X}=0$ for $i>d$, in other words $H^{i}\left(f_{+}^{(m)} \omega_{\mathscr{C}, \mathrm{Q}}\left[d_{X}\right]\right)=0$ 
for $i>-d_{Y}$. Thus, we get

$$
\operatorname{Hom}\left(f_{+}^{(m)} \omega_{X}\left[d_{X}\right], \omega_{Y}\left[d_{Y}\right]\right) \cong \operatorname{Hom}\left(H^{d} f_{+}^{(m)} \omega_{X}, \omega_{Y}\right) .
$$

This implies that to give the trace $\operatorname{map} f_{+}^{(m)} \omega_{X}\left[d_{X}\right] \rightarrow \omega_{Y}\left[d_{Y}\right]$ is equivalent to giving the homomorphism $H^{d} f_{+}^{(m)} \omega_{X} \rightarrow \omega_{Y}$. We can retrieve the trace map by the composition

$$
f_{+}^{(m)} \omega_{X}\left[d_{X}\right] \rightarrow H^{d} f_{+}^{(m)} \omega_{X}\left[d_{Y}\right] \rightarrow \omega_{Y}\left[d_{Y}\right] .
$$

Note that we have the following commutative diagram by [Vi2, III, 5.4].

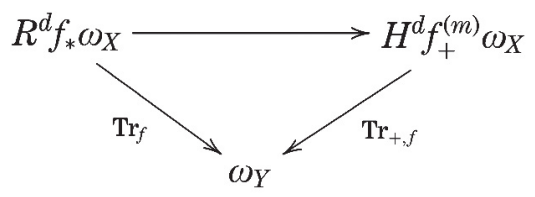

It suffices to see that the following diagram is commutative.

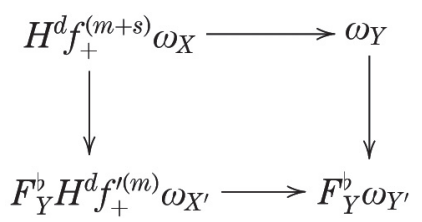

This shows that the problem is local with respect to $Y$. Assume that there exist liftings $F_{X}: X \rightarrow X^{\prime}$ and $F_{Y}: Y \rightarrow Y^{\prime}$ of relative Frobenius morphisms such that the two morphisms $f^{\prime} \circ F_{X}, F_{Y} \circ f: X \rightarrow Y^{\prime}$ coincide. Under this particular situation, the theorem is reduced to showing the following diagram

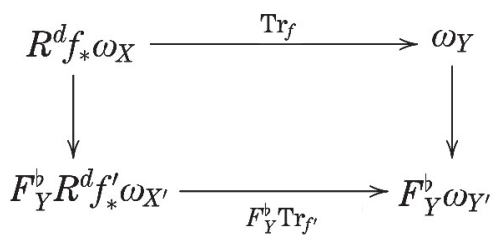

is commutative, where the left vertical homomorphism is the base change homomorphism. This is nothing but (4.12.1). In particular, the theorem holds in the case where $f$ is finite étale.

Let $x$ be a point of $X$ (which may not be closed). For a right $\mathscr{D}_{X}^{(m)}$ module $\mathscr{F}$ and an integer $i$, we put

$$
\mathscr{H}_{x}^{(m), i}(\mathscr{F}):={\underset{x \in U}{\longrightarrow}}_{\lim _{U *}} R^{i} \underline{\Gamma}_{U \cap \overline{\{x\}}}^{(m)}(\mathscr{F})
$$


Explicit calculation of Frobenius isomorphisms and Poincaré duality etc. 135

where $U$ runs over open neighborhoods of $x, j_{U}: U \hookrightarrow X$ is the inclusion, and $\underline{\Gamma}^{(m)}$ denotes the level $m$ local cohomology functor defined in [BeI, 4.4.4]. We note that

$$
\underset{m^{\prime}}{\lim } \mathscr{H}_{x}^{\left(m^{\prime}\right), i}(\mathscr{F}) \cong \mathscr{H}_{x}^{i}(\mathscr{F})
$$

by [Be1, 1.5.4], and $\mathscr{H}_{x}^{i}\left(\omega_{X}\right)$ can be seen as a quasi-coherent right $\mathscr{D}_{X}^{(m)}$-module. The Frobenius isomorphism $\mathscr{H}_{x}^{(m+s), i}\left(\omega_{X}\right) \stackrel{\sim}{\rightarrow} F_{X}^{b} \mathscr{H}_{x}^{(m), i}\left(\omega_{X^{\prime}}\right)$ induces the isomorphism

$$
\phi_{x}^{i}: \mathscr{H}_{x}^{i}\left(\omega_{X}\right) \stackrel{\sim}{\rightarrow} F_{X}^{b} \mathscr{H}_{x}^{i}\left(\omega_{X^{\prime}}\right) .
$$

This induces the following isomorphism of Cousin complexes (cf. [Ha, IV, §3]).

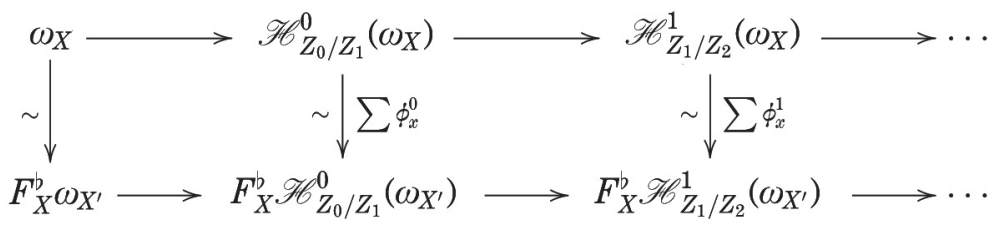

We denote by $\mathscr{C}_{X}^{\bullet}$ the Cousin complex of $\omega_{X}$.

Let $y$ be a point of codimension $i$ in $Y$, and let $x$ be a closed point of the fiber $f^{-1}(y)$ in $X$. For closed subsets of schemes, let us endow with the reduced induced scheme structure. Then since $f$ is smooth, there exists an open subscheme $U$ of $X$, such that $f^{\prime}: Z:=\overline{\{x\}} \cap U \rightarrow W:=\overline{\{y\}} \cap V$ is finite étale where $V:=f(U)$, and $W$ is smooth. Consider the following commutative diagram.

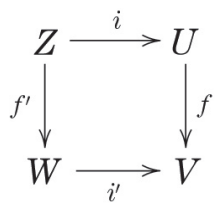

The trace map $\operatorname{Tr}_{+, f^{\prime}}: f_{+}^{\prime(m)} \omega_{Z} \rightarrow \omega_{W}$ can be identified with the usual trace map $\operatorname{Tr}_{f^{\prime}}$ by the isomorphism $f_{*}^{\prime} \cong f_{+}^{\prime}$ since $f^{\prime}$ is finite étale. Since $\mathscr{H}_{Z}^{(m), i}\left(\omega_{X}\right) \cong i_{+}^{(m)}\left(\omega_{Z}\right)$ and $\mathscr{H}_{W}^{(m), i}\left(\omega_{Y}\right) \cong i_{+}^{\prime(m)}\left(\omega_{W}\right)$, the functor $i_{+}^{\prime(m)} \circ \mathrm{Tr}_{+, f^{\prime}}$ induces the homomorphism

$$
H^{0} f_{+}^{(m)} \mathscr{H}_{x}^{(m), d+i}\left(\omega_{X}\right) \rightarrow \mathscr{H}_{y}^{(m), i}\left(\omega_{Y}\right)
$$

by taking inductive limit over $V$. Note that since $f^{\prime}$ is finite étale, this trace map is compatible with Frobenius by the result of the first part of this proof. By taking the inductive to (4.13.2) over $m$ and using the identification 
(4.13.1), we get a homomorphism

$$
\begin{aligned}
& \operatorname{Tr}_{x}^{i}: H^{0} f_{+}^{(m)} \mathscr{H}_{x}^{d+i}\left(\omega_{X}\right) \cong \underset{m^{\prime}}{\lim } H^{0} f_{+}^{(m)} \mathscr{H}_{x}^{\left(m^{\prime}\right), d+i}\left(\omega_{X}\right) \\
& \rightarrow \underset{m^{\prime}}{\lim } H^{0} f_{+}^{\left(m^{\prime}\right)} \mathscr{H}_{x}^{\left(m^{\prime}\right), d+i}\left(\omega_{X}\right) \stackrel{(4.13 .2)}{\longrightarrow} \underset{m^{\prime}}{\lim } \mathscr{H}_{y}^{\left(m^{\prime}\right), i}\left(\omega_{Y}\right) \cong \mathscr{H}_{y}^{i}\left(\omega_{Y}\right) .
\end{aligned}
$$

This homomorphism is compatible with Frobenius as well since (4.13.2) is. The composition

$$
f_{*} \mathscr{H}_{x}^{d+i}\left(\omega_{X}\right) \rightarrow H^{0} f_{+}^{(m)} \mathscr{H}_{x}^{d+i}\left(\omega_{X}\right) \stackrel{\operatorname{Tr}_{x}^{i}}{\longrightarrow} \mathscr{H}_{y}^{i}\left(\omega_{Y}\right)
$$

is the usual trace map by construction.

There exists the surjection $\mathscr{D}_{X}^{(m)} \rightarrow \mathscr{D}_{X \rightarrow Y}^{(m)}$ sending 1 to $1 \otimes 1$. This gives us a flat resolution $\mathscr{D}^{\bullet} \rightarrow \mathscr{D}_{X \rightarrow Y}^{(m)}$ such that $\mathscr{L}^{0}:=\mathscr{D}_{X}^{(m)}$ and $\mathscr{L}^{i}=0$ for $i>0$. The double complex $f_{*}\left(\mathscr{C}_{X}^{\bullet} \otimes_{\mathscr{D}_{X}^{(m)}} \mathscr{L}^{\bullet}\right)$ induces the spectral sequence

$$
E_{1}^{a, b}=f_{*} H_{-b}\left(\mathscr{H}_{Z_{a} / Z_{a+1}}^{a}\left(\omega_{X}\right) \otimes^{\mathrm{L}} \mathscr{D}_{X \rightarrow Y}^{(m)}\right) \Rightarrow H^{a+b} f_{+}^{(m)} \omega_{X}
$$

Note that $E_{1}^{a, b} \cong H_{-b} f_{+}^{(m)} \mathscr{H}_{Z_{a} / Z_{a+1}}^{a}\left(\omega_{X}\right)$. The trace map (4.13.4) $E_{1}^{d+i, 0} \rightarrow$ $\mathscr{H}_{Z_{i} / Z_{i+1}}\left(\omega_{Y}\right)$ induces the homomorphism of complexes $f_{*}\left(\mathscr{C}_{X}^{d+i} \otimes \mathscr{B}_{\bullet}\right) \rightarrow$ $\mathscr{H}_{Z_{i} / Z_{i+1}}^{i}\left(\omega_{Y}\right)=\mathscr{C}_{Y}^{i}$, and this induces the homomorphism of double complexes

$$
f_{*}\left(\mathscr{C}_{X}^{d+\bullet} \otimes \mathscr{D}^{\bullet}\right) \rightarrow \mathscr{C}_{Y}^{\bullet}
$$

This homomorphism defines the homomorphism $\gamma: f_{+} \omega_{X}\left[d_{X}\right] \rightarrow \omega_{Y}\left[d_{Y}\right]$. Let us show that $\gamma=\operatorname{Tr}_{+, f}$. The canonical homomorphism $\mathscr{C}_{X}^{\bullet} \rightarrow \mathscr{C}_{X}^{\bullet} \otimes \mathscr{B}^{0}$ induces the homomorphism of double complexes

$$
f_{*} \mathscr{C}_{X}^{\bullet} \rightarrow f_{*}\left(\mathscr{C}_{X}^{d+\bullet} \otimes \mathscr{B}^{\bullet}\right)
$$

Let

$$
{ }_{\mathrm{I}} E_{1}^{a, b}:=\left\{\begin{array}{ll}
f_{*} \mathscr{C}_{X}^{a} & \text { if } b=0 \\
0 & \text { if } b \neq 0
\end{array} \quad{ }_{\mathrm{II}} E_{1}^{a+d, b} \quad:= \begin{cases}\mathscr{C}_{Y}^{a} & \text { if } b=0 \\
0 & \text { if } b \neq 0\end{cases}\right.
$$

Then we get the trivial spectral sequences ${ }_{\mathrm{I}} E_{1}^{a, b} \Rightarrow R^{a+b} f_{*} \omega_{X}$ and ${ }_{\mathrm{II}} E_{1}^{a, b} \Rightarrow{ }_{\mathrm{II}} E^{n}$ where ${ }_{\mathrm{II}} E^{d}:=\omega_{Y}$ and 0 otherwise. The homomorphisms (4.13.5) and (4.13.6) induce the homomorphisms ${ }_{\mathrm{I}} E_{1}^{a, b} \rightarrow E_{1}^{a, b} \rightarrow \mathrm{II}_{1}^{a, b}$ of spectral sequences. We get the following homomorphisms of complexes of $E_{1}$-terms of these spectral sequences. 


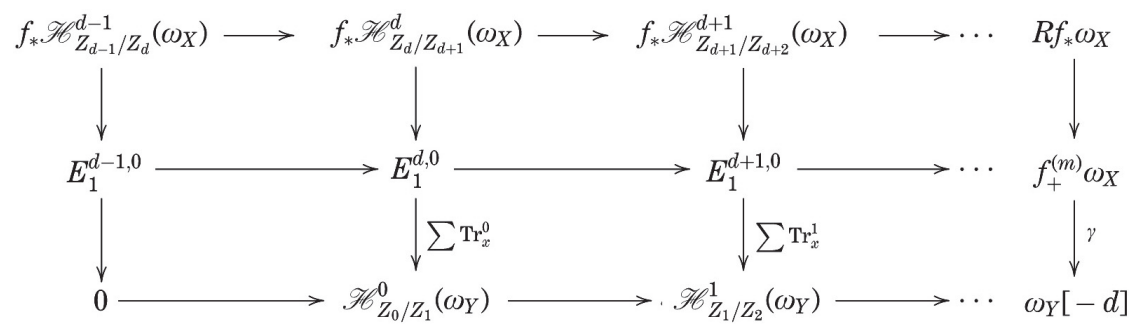

Here the right homomorphisms are the homomorphisms of complexes of corresponding spectral sequences. To show that $\gamma$ is the trace map, it suffices to show that $H^{-d_{Y}}(\gamma)$ is the trace map. Consider the homomorphisms

$$
R^{d} f_{*} \omega_{X} \rightarrow H^{d} f_{+}^{(m)} \omega_{X} \stackrel{H^{d} \gamma}{\longrightarrow} \omega_{Y}
$$

induced by the $E^{d}$-terms of the homomorphism of the spectral sequences. The composition is the usual trace map $\operatorname{Tr}_{f}$ since (4.13.4) is (cf. [Ha, VI, 4.2] for the construction of the classical trace map). Moreover the first homomorphism is the natural map which is surjective. Thus the second homomorphism is nothing but the trace map of Virrion.

Since it suffices to see the Frobenius compatibility for $H^{d} \gamma$, this shows that it suffices to show the Frobenius compatibility for the lower homomorphism of complexes of the diagram (4.13.7). Thus it is reduced to showing the Frobenius compatibility for the homomorphism

$$
\sum \operatorname{Tr}_{x}^{i}: E_{1}^{d+i, 0} \rightarrow \mathscr{H}_{Z_{i} / Z_{i+1}}^{i}\left(\omega_{Y}\right)
$$

for any $i$. It is enough to show the compatibility for $\operatorname{Tr}_{x}^{i}$ for each $x \in X$ and $i$, which we have already verified at (4.13.3).

4.14. For most of the applications, the assumption of Proposition 4.13 is not too strong, but for the sake of completeness, we will eliminate the assumption on the factorization.

Let $\mathscr{H}$ be a smooth formal scheme. We say that a smooth $\mathscr{H}$-scheme $X$ (or the morphism $X \rightarrow \mathscr{H}$ ) where $X$ is a scheme over $k$ is $\mathscr{H}$-embeddable if there exists a smooth formal scheme $\mathscr{P}$ proper and smooth over $\mathscr{Y}$, and a closed immersion $X \hookrightarrow \mathscr{P}$ over $\mathscr{Y}$. Given a $\mathscr{Y}$-embeddable morphism, we can also consider the trace homomorphism. The category $F-D_{\text {overcoh }}^{b}\left(\mathscr{D}_{X, Q}^{\dagger}\right)$ of overcoherent $F-\mathscr{D}_{X, Q}^{\dagger}$-complexes (cf. [Ca1, 3.1.1]) is the subcategory of the overcoherent $F-\mathscr{D}_{\mathscr{P}, Q}^{\dagger}$-complexes consisting of 
complexes with support in $X$. This does not depend on the choice of $\mathscr{P}$ (cf. [Ca6, 3.12]). Given a $\mathscr{Y}$-embeddable homomorphism $f: X \rightarrow \mathscr{Y}$, we have a push-forward functor

$$
f_{+}: F-D_{\text {overcoh }}^{b}\left(\mathscr{D}_{X, Q}^{\dagger}\right) \rightarrow F-D_{\text {overcoh }}^{b}\left(\mathscr{D}_{\mathscr{Y}, Q}^{\dagger}\right),
$$

which is nothing but the push-forward by the proper morphism $\mathscr{P} \rightarrow \mathscr{Y}$.

Let $g: X \hookrightarrow \mathscr{P}$ be a closed immersion, and we put $\omega_{X}:=R \underline{\Gamma}_{X}^{\dagger}\left(\omega_{\mathscr{P}}\right)\left[-d_{X} / \mathscr{P}\right]$ where $d_{X / \mathscr{P}}:=\operatorname{dim}(X)-\operatorname{dim}(\mathscr{P})$. This does not depend on the choice of the embedding by $[\mathrm{Ca} 6,3.16]$. We define the trace homomorphism $\operatorname{Tr}_{+, g}: g_{+} \omega_{X}\left[d_{X}\right] \rightarrow \omega_{\mathscr{P}}\left[d_{P}\right]$ to be the homomorphism induced by the canonical homomorphism $R \underline{\Gamma}_{X}^{\dagger}\left(\omega_{\mathscr{P}}\right) \rightarrow \omega_{\mathscr{P}}$. Of course, this homomorphism is compatible with Frobenius structure. When $X$ can be lifted to a smooth formal scheme $\mathscr{X}, \omega_{X}$ is canonically isomorphic to $\omega_{\mathscr{X}}$, and this trace homomorphism coincides with the usual one by [Ca7, 2.2.7].

Now, let $f: X \rightarrow \mathscr{Z}$ be a $\mathscr{F}$-embeddable morphism. Take a factorization $\left(g, f^{\prime}\right): X \stackrel{g}{\rightarrow} \mathscr{P} \stackrel{f^{\prime}}{\rightarrow} \mathscr{Y}$ of $f$ where $g$ is a closed immersion and $f^{\prime}$ is proper (not necessarily smooth). Then we define the trace homomorphism by

$$
\operatorname{Tr}_{+,\left(g, f^{\prime}\right)}:=\operatorname{Tr}_{+, f^{\prime}} \circ f_{+}^{\prime}\left(\operatorname{Tr}_{+, g}\right): f_{+} \omega_{X}\left[d_{X}\right] \rightarrow \omega_{\mathscr{Y}}\left[d_{Y}\right] .
$$

Lemma. (i) The homomorphism $\operatorname{Tr}_{+,\left(g, f^{\prime}\right)}$ does not depend on the choice of factorizations. We denote this trace homomorphism by $\operatorname{Tr}_{+, f}$.

(ii) Let $f: X \rightarrow \mathscr{H}$ be a proper morphism over $R$ where $X$ is smooth over $k$ and $\mathscr{Y}$ is a smooth formal scheme, and $g: \mathscr{F} \rightarrow \mathscr{Z}$ be a proper morphism between smooth formal schemes. Assume that $g \circ f$ is $\mathbb{Z}$-embeddable. Then $f$ is $\mathscr{Y}$-embeddable as well, and $\operatorname{Tr}_{+, g} \circ g_{+}\left(\operatorname{Tr}_{+, f}\right) \cong \operatorname{Tr}_{+, g} \circ f$.

Proof. Let us prove (i). Let $\left(g_{P}, f_{P}^{\prime}\right): X \rightarrow \mathscr{P} \rightarrow \mathscr{Y}$ be a factorization such that $f_{P}^{\prime}$ is proper smooth, and $\left(g_{Q}, f_{Q}^{\prime}\right): X \rightarrow Q \rightarrow \mathscr{Y}$ be a factorization such that $f_{Q}^{\prime}$ is proper. Consider the following commutative diagram:

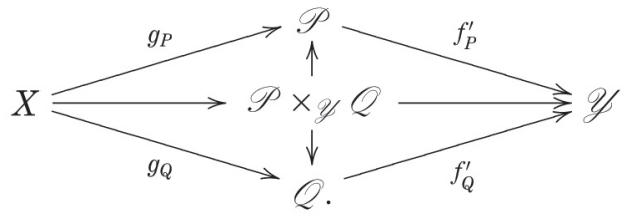

We note that the product $\mathscr{P} \times \mathscr{Q} \mathcal{Q}$ is a smooth formal scheme since $f_{P}^{\prime}$ is smooth, and this is proper over $\mathscr{Y}$. By the transitivity of the trace homomorphism of Virrion [Vi2, III, 5.5], it suffices to show the following: given a 
commutative diagram

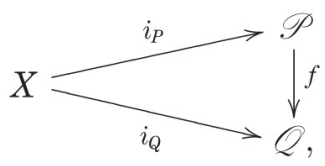

where $X$ is smooth over $k, \mathscr{P}$ and $\mathscr{Q}$ are smooth formal schemes, $i_{P}$ and $i_{Q}$ are closed immersions, and $f$ is proper, the following diagram is commutative:

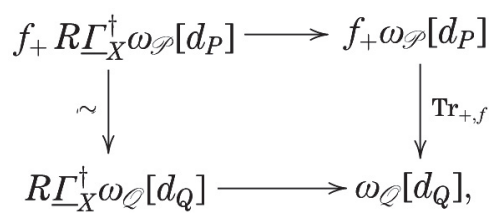

where the horizontal homomorphisms are canonical ones and the left vertical homomorphism is the isomorphism of Caro [Ca6, 3.12]. Let us prove

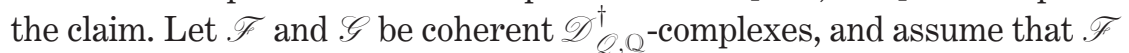
is supported on $X$. Then we see that

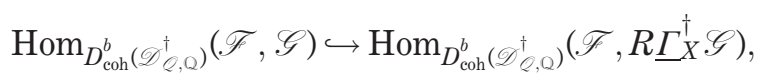

in the same way as the proof of [Ca7, 2.2.8]. Thus, it suffices to see the commutativity after taking $R \Gamma_{X}^{\dagger}$. Then since all the complexes are concentrated in degree $-\operatorname{dim}(X)$, the verification is local, and we may assume that $X$ is liftable. In this case, the claim holds by the transitivity of trace homomorphism by Virrion.

Let us prove (ii). Let $(a, b): X \rightarrow \mathscr{Q} \rightarrow \mathscr{Z}$ be a factorization of $g \circ f$ such that $b$ is proper and smooth. Consider the following commutative diagram:

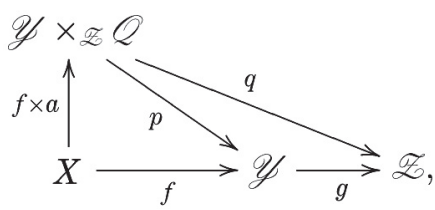

where $p$ and $q$ are the projections. The morphism $p$ is proper smooth since $b$ is. Considering (i) and the transitivity of the trace homomorphism by Virrion, we get (ii).

By definition and Proposition 4.13, $\operatorname{Tr}_{+,\left(g, f^{\prime}\right)}$ is compatible with Frobenius structure when $f^{\prime}$ is smooth, and thus the lemma above shows that $\operatorname{Tr}_{+, f}$ is compatible with Frobenius structure. 
4.15. Let $f: X \rightarrow \mathscr{Y}$ be a $\mathscr{Y}$-embeddable morphism, and assume that the induced homomorphism of the special fibers $X \rightarrow Y$ is an alteration. Then we are able to define a homomorphism $\alpha: \omega_{Y} \rightarrow f_{+} \omega_{X}$ in the following way. We claim that

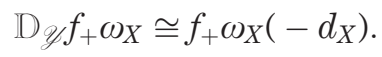

Indeed, take a factorization $\left(g, f^{\prime}\right): X \rightarrow \mathscr{P} \rightarrow \mathscr{Y}$ where $f^{\prime}$ is proper smooth. By definition and Proposition 4.13, we get $\mathrm{D}_{\mathscr{Y} f_{+}} \omega_{X} \cong f_{+}^{\prime} \mathrm{D}_{\mathscr{P}} R \Gamma_{X}^{\dagger}\left(\omega_{\mathscr{P}}\right)$, and it suffices to show that

$$
\mathrm{D}_{\mathscr{P}} R \underline{\Gamma}_{X}^{\dagger}\left(\omega_{\mathscr{P}}\right) \cong R \underline{\Gamma}_{X}^{\dagger}\left(\omega_{\mathscr{P}}\right)\left(-d_{X}\right)
$$

If we forget about the Frobenius structure, we have $\mathbb{D}_{\mathscr{P}} R \Gamma_{X}^{\dagger}\left(\omega_{\mathscr{P}}\right) \cong$ $R \underline{\Gamma}_{X}^{\dagger}\left(\omega_{\mathscr{P}}\right)$, which is concentrated at degree $-d_{X / \mathscr{P}}$. When $X$ is liftable, we get (4.15.2) by 3.11, so we get the same isomorphism even when $X$ is not liftable since it is concentrated at one degree, and we get the isomorphism (4.15.1). We see easily that this isomorphism does not depend on the choice of the factorization.

Now, we define

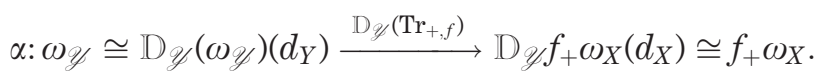

By the construction, $\alpha$ does not depend on the choice of factorizations. Since $\mathrm{D}\left(\mathrm{Tr}_{+, f}\right)$ is compatible with Frobenius, $\alpha$ is compatible as well.

Finally, if moreover the morphism $X \rightarrow Y$ is generically étale, we have $\operatorname{Tr}_{+, f} \circ \alpha=\operatorname{deg}(f)$. Indeed, a homomorphism $\omega_{y} \rightarrow \omega_{y}$ is determined generically, and since $f$ is generically finite étale, the claim follows.

4.16. THEOREM. We preserve the assumptions and notation of paragraph 4.11. Then the relative duality isomorphism (4.11.2) is compatible with Frobenius pull-back.

Proof. Let $X$ be the special fiber of $\mathscr{C}$. There exists a generically étale alteration $g: X^{\prime} \rightarrow X$ and an open immersion $X^{\prime} \hookrightarrow \overline{X^{\prime}}$ such that $\overline{X^{\prime}}$ is smooth projective over $k$. Take a closed immersion $\overline{X^{\prime}} \hookrightarrow \mathbb{P}^{n}$ and let $i: X^{\prime} \hookrightarrow \overline{X^{\prime}} \hookrightarrow \mathbb{P}^{n}$. We have the following factorization

$$
X^{\prime} \stackrel{i \times g}{\longrightarrow} \widehat{\mathbb{P}}^{n} \times \mathscr{C} \rightarrow \mathscr{C},
$$

where $i \times g$ is a closed immersion since $g$ is proper. Thus, $X^{\prime}$ is $\mathscr{C}$-embeddable. In the same way, $X^{\prime}$ is $\mathscr{Y}$-embeddable as well. Since $X^{\prime}$ is $\mathscr{C}$ - 
Explicit calculation of Frobenius isomorphisms and Poincaré duality etc. 141

embeddable, the trace homomorphism $\operatorname{Tr}_{+, g^{\prime}}: g_{+}^{\prime} \omega_{X^{\prime}} \rightarrow \omega_{\mathscr{X}}$ is compatible with Frobenius. Since $g^{\prime}$ is a generically étale alteration, we also get a homomorphism $\alpha: \omega_{\mathscr{X}} \rightarrow g_{+}^{\prime} \omega_{X}$ compatible with Frobenius by paragraph 4.15, and the composition $\mathrm{Tr}_{+, g^{\prime}} \circ \alpha$ is equal to the multiplication by $\operatorname{deg}\left(g^{\prime}\right)$. On the other hand, since $X^{\prime}$ is $\mathscr{Y}$-embeddable, $\operatorname{Tr}_{+, f \circ g^{\prime}}$ is compatible with Frobenius. Consider the following homomorphisms

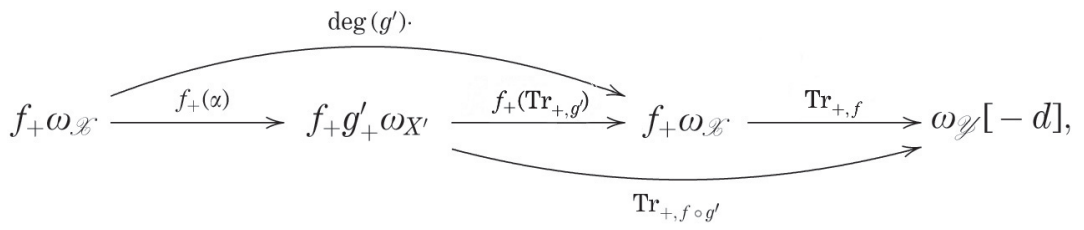

where $d:=d_{X}-d_{Y}$. The composition of these homomorphisms is equal to $\operatorname{deg}\left(g^{\prime}\right) \cdot \operatorname{Tr}_{+, f}$. Since $f_{+}(\alpha)$ and $\operatorname{Tr}_{+, f \circ g^{\prime}}$ are compatible with Frobenius, the composition deg $\left(g^{\prime}\right) \cdot \operatorname{Tr}_{+, f}$ is compatible as well, and thus $\operatorname{Tr}_{+, f}$ is compatible with Frobenius, which completes the proof.

4.17. We preserve the assumptions and notation from paragraph 4.11. Let $\mathscr{C}^{\prime}$ be an object in $D_{\mathrm{coh}}^{b}\left(\mathscr{D}_{\mathscr{C}^{\prime}, \mathrm{Q}}^{\dagger}{ }^{\dagger} Z\right)$ ), and $\mathscr{N}^{\prime}$ be an object in $D_{\mathrm{coh}}^{b}\left(\mathscr{D}_{\mathscr{Y}^{\prime}, \mathrm{Q}}^{\dagger}\left({ }^{\dagger} W\right)\right)$. We put $\mathscr{C}:=F_{X}^{*} \mathscr{C l}$ and $\mathscr{N}:=F_{X}^{*} \mathscr{N}^{\prime}$. Since $F_{Y}^{*}$ induces an equivalence between $D_{\mathrm{coh}}^{b}\left(\mathscr{D}_{\mathscr{Y}^{\prime}, \mathrm{Q}}^{\dagger}\left({ }^{\dagger} W\right)\right)$ and $D_{\mathrm{coh}}^{b}\left(\mathscr{D}_{\mathscr{Y}, \mathrm{Q}}^{\dagger}\left({ }^{\dagger} W\right)\right)$, we get

$$
\begin{aligned}
R \operatorname{Hom}_{\mathscr{Q}_{\mathscr{Y}, Q}^{\dagger}\left({ }^{\dagger} W\right)}\left(f_{+}(\mathscr{C O}), \mathscr{N}\right) & \stackrel{\sim}{\rightarrow} R \operatorname{Hom}_{\mathscr{D}_{\mathscr{Y}, Q}^{\dagger}\left({ }^{\dagger} W\right)}\left(F_{Y}^{*} f_{+}\left(\mathscr{C l}^{\prime}\right), F_{Y}^{*} \mathscr{N}^{\prime}\right) \\
& \stackrel{\sim}{\rightarrow} R \operatorname{Hom}_{\mathscr{D}_{\mathscr{Y}^{\prime}, Q}^{\dagger}\left({ }^{\dagger} W\right)}\left(f_{+}^{\prime}\left(\mathscr{C l}^{\prime}\right), \mathscr{N}^{\prime}\right)
\end{aligned}
$$

where the first isomorphism is induced by the isomorphism of functors $F_{Y}^{*} \circ f_{+}^{\prime} \cong f_{+} \circ F_{X}^{*}$. In the same way, we get an isomorphism

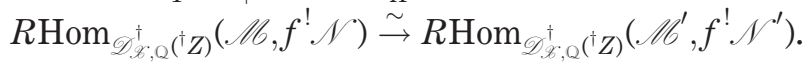

COROLlary. We preserve the assumptions and notation. The adjoint isomorphism is compatible with Frobenius, in other words, the following diagram is commutative.

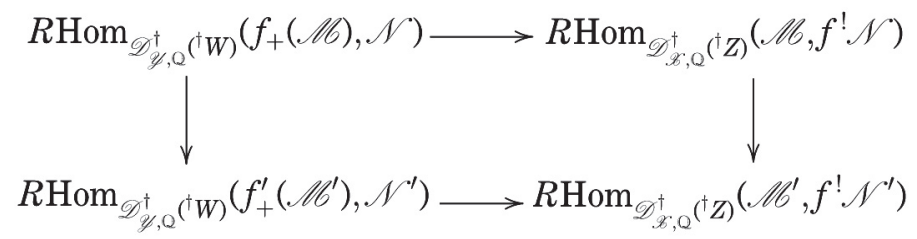


Here the horizontal isomorphism are the adjoint formula isomorphism [Vi, IV, 4.2], and the vertical isomorphisms are those we have just defined.

Proof. We only need to check the compatibility with Frobenius of the isomorphisms used in the proof of [Vi2, IV, 4.1]. The compatibility of the isomorphism [Vi2, IV, 1.1 (i)] is nothing but [Ca3, 2.1.19]. The compatibility of [Vi2, IV, 3.4] is Theorem 4.16.

\section{Cohomological operations in arithmetic $\mathscr{D}$-modules}

In this last section, we will collect results on six operations in the theory of arithmetic $\mathscr{D}$-modules with Frobenius structures in the liftable case. Before starting, recall the notation and terminologies of paragraph 3.1. We refer to [Ab2] for thorough treatment in this direction.

In this section, any (formal) scheme is assumed to be of finite type over its basis.

5.1. In this section, we consider the situation 2.1 .1 if we do not consider the Frobenius structure, and 2.1.2 if we use modules with Frobenius structure. Let $f:(\mathscr{C}, Z) \rightarrow(\mathscr{Y}, W)$ be a morphism of d-couples. We put $Z^{\prime}:=f^{-1}(Z) \subset Z$, which is a divisor by the definition of morphisms of d-couples. For a coherent $(F-) \mathscr{D}_{\mathscr{Y}, \mathrm{Q}}^{\dagger}\left({ }^{\dagger} W\right)$-complex $\mathscr{C l}$, recall that

$$
f^{!} \mathscr{\mathscr { C }}:=\mathscr{D}_{\mathscr{X}, \mathrm{Q}}^{\dagger}\left({ }^{\dagger} Z\right) \otimes_{\mathscr{D}_{\mathscr{X}, \mathrm{Q}}^{\dagger}\left(^{\dagger} Z^{\prime}\right)} f_{0}^{!}(\mathscr{C O})
$$

in $(F-) \stackrel{L D}{\mathrm{Q}, \mathrm{qc}}_{\mathrm{b}}\left(\widehat{\mathscr{D}}_{\mathscr{O}}^{(\bullet)}(Z)\right)$ (cf. paragraph 3.1). Also recall that we denote by $\mathbb{D}_{\mathscr{X}, Z}$ the dual functor with respect to $(F-) \mathscr{D}_{\mathscr{C}, Q}^{\dagger}\left({ }^{\dagger} Z\right)$-modules. Let $\mathscr{C}$ be a coherent $(F-) \mathscr{D}_{\mathscr{Y}, \mathrm{Q}}^{\dagger}\left({ }^{\dagger} W\right)$-module (or perfect complex), and suppose that $f ! \circ \mathbb{D}_{\mathscr{Y}, W}(\mathscr{C O})$ is a perfect complex. Then we put

$$
f^{+}(\mathscr{C O}):=\left(\mathbb{D}_{\mathscr{X}, Z} \circ f^{!} \circ \mathbb{D}_{\mathscr{Y}, W}\right)(\mathscr{C O})
$$

in $(F-) D_{\text {coh }}^{b}\left(\mathscr{D}_{\mathscr{C}, \mathrm{Q}}^{\dagger}\left({ }^{\dagger} Z\right)\right)$. When the realization of $f$ is smooth, this functor is defined for any perfect $(F-) \mathscr{D}_{\mathscr{Y}, \mathrm{Q}}^{\dagger}\left({ }^{\dagger} W\right)$-complexes by [BeI, 4.3.3]. If Berthelot's conjecture (cf. [BeI, 5.3.6]) is valid, this functor is defined for any holonomic $F$ - $\mathscr{D}_{\mathscr{Y}, \mathrm{Q}}^{\dagger}\left({ }^{\dagger} W\right)$-complexes.

5.2. Let $f:(\mathscr{C}, Z) \rightarrow(\mathscr{Y}, W)$ be a morphism of d-couples such that the realization is proper. Let $Z^{\prime}:=f^{-1}(W) \subset Z$. We denote by $f_{0, Z^{\prime},+}$ the proper

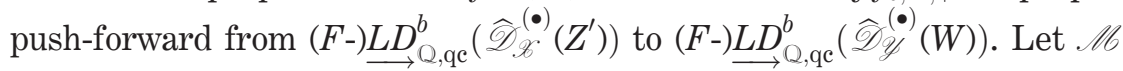


Explicit calculation of Frobenius isomorphisms and Poincaré duality etc. 143

be a coherent $\mathscr{D}_{\mathscr{C}, Q}^{\dagger}\left({ }^{\dagger} Z\right)$-complex. We denote by $j_{+} \mathscr{C l}$ the underlying $\mathscr{D}_{\mathscr{C}, Q}^{\dagger}\left({ }^{\dagger} Z^{\prime}\right)$-complex of $\mathscr{C}$. We define

$$
f_{+}(\mathscr{C l}):=f_{0, Z^{\prime},+}\left(j_{+} \mathscr{C l}\right)
$$

in $\underline{L D}_{\mathrm{Q}, \mathrm{qc}}^{b}\left(\widehat{\mathscr{D}}_{\mathscr{Y}}^{(\bullet)}(W)\right)$. Let $\mathscr{C}$ be a perfect $\left.(F-)_{\mathscr{D}, \mathrm{Q}}^{\dagger}{ }^{\dagger} Z\right)$-complex such that $\mathbb{D}_{\mathscr{C}, Z}(\mathscr{C})$ is a coherent $(F-) \mathscr{D}_{\mathscr{C}, Q}^{\dagger}\left({ }^{\dagger} Z^{\prime}\right)$-complex. In this case, we say that $\mathscr{C}$ is $f_{!}$-admissible. Then we define

$$
f_{!}(\mathscr{C l}):=\left(\mathbb{D}_{\mathscr{Y}, W} \circ f_{+} \circ \mathrm{D}_{\mathscr{C}, Z}\right)(\mathscr{C b})
$$

in $(F-) D_{\mathrm{coh}}^{b}\left(\mathscr{D}_{\mathscr{y}, \mathrm{Q}}^{\dagger}\left({ }^{\dagger} W\right)\right)$. When $Z^{\prime}=Z$, any perfect complex is $f_{!}$-admissible. If Berthelot's conjecture is valid, any holonomic module is $f_{\text {!-admissible. }}$ Another example we have in mind is the geometric Fourier transform [NH1] or that with compact support.

5.3. Let $(\mathscr{C}, Z)$ be a d-couple. For coherent $\mathscr{D}_{\mathscr{C}, \mathrm{Q}}^{\dagger}\left({ }^{\dagger} Z\right)$-modules $\mathscr{C}$ and $\mathscr{N}$, we denote $\mathscr{\mathscr { C }}{\left.\stackrel{\mathrm{L}}{\otimes}{ }_{\mathcal{O}, Q}^{\dagger}{ }^{\dagger} Z\right)^{N}}^{N}$ simply by $\mathscr{\mathscr { C }} \stackrel{\mathrm{L}}{\otimes}^{\dagger} \mathscr{N}$. This is an object in $\underset{L D_{Q, q c}^{b}}{b}\left(\widehat{\mathscr{D}}_{\mathscr{C}}^{(\bullet)}(Z)\right)$. Now, let $f$ be a morphism of d-couples. We have defined functors $\stackrel{L}{\otimes} \dagger^{\dagger}, D_{\mathscr{C}, Z}$, and $f_{+}, f_{!}, f^{+}, f^{!}$. These functors are expected to fit in the framework of six functors if we consider the category of holonomic complexes. Let us explain this shortly. Consider the category dcouples such that the morphisms consist of strict morphisms of d-couples (cf. paragraph 3.1) whose realizations are proper. For a d-couple $(\mathscr{B}, Z)$, we consider the category of holonomic $F-\mathscr{D}_{\mathscr{X}, Q}^{\dagger}\left({ }^{\dagger} Z\right)$-complexes denoted by $\mathcal{C}_{(\mathscr{C}, \mathcal{Z})}$. If the Berthelot conjecture holds, the category $\mathcal{C}_{(\mathscr{X}, \mathcal{Z})}$ is stable under six operations. Philosophically, considering $\mathcal{C}_{(\mathscr{C}, \mathcal{Z})}$ means to consider a "good category" of coefficients on $\mathscr{C} \backslash Z$. See [BeI, 5.3.6] for some explanations. The following theorems are stating that fundamental relations of these functors hold in this framework.

5.4. We use the notation of paragraph 5.2. We get the following theorem.

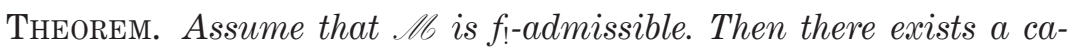
nonical homomorphism

$$
f_{!}(\mathscr{C l}) \rightarrow f_{+}(\mathscr{C O})
$$

compatible with Frobenius pull-backs. Moreover, when $Z^{\prime}=Z$, this homomorphism is an isomorphism. 
Proof. By using Theorem 4.16, we get $\mathbb{D}_{\mathscr{Y}, W} \circ f_{Z^{\prime},+} \cong f_{Z^{\prime},+} \circ \mathbb{D}_{\mathscr{K}, Z^{\prime}}$. The extension of scalar $\mathscr{D}_{\mathscr{X}, Q}^{\dagger}\left({ }^{\dagger} Z\right) \otimes_{\mathscr{P}_{X, Q}^{\dagger},\left(Z^{\prime}\right)}$ induces the functor $\mathrm{D}_{\mathscr{X}, Z^{\prime}} \circ j_{+} \rightarrow$ $j_{+} \circ \mathrm{D}_{\mathscr{X}, Z}$. Using the isomorphism $\mathrm{D}_{\mathscr{K}, Z} \circ \mathrm{D}_{\mathscr{X}, Z} \cong$ id compatible with the Frobenius isomorphisms by [Vi, II, 3.5], and combining these morphisms of functors we get the homomorphism (5.4.1). The latter assertion is now clear.

5.5. Now, we will show the Poincaré duality theorem for arithmetic $\mathscr{D}$ module theory in the style of SGA4 Exp. XVIII Théorème 3.2.5.

Let $f:(\mathscr{C}, Z) \rightarrow(\mathscr{Y}, W)$ be a strict morphism of d-couples such that the realization is proper. Moreover, we assume that $f_{0}^{-1}(W)=Z$. There exists the following isomorphism thanks to [Vi2, IV, 7.4].

$$
\left.R \operatorname{Hom}_{\mathscr{Q} \mathscr{Q}, Q}^{\dagger}{ }^{\dagger} Z\right)\left(\mathscr{L l}, f^{!} \mathscr{N}\right) \stackrel{\sim}{\rightarrow} R \operatorname{Hom}_{\mathscr{Y}_{Y, Q}^{\dagger}(\dagger W)}\left(f_{+} \mathscr{L l}, \mathscr{N}\right)
$$

We get that this isomorphism is compatible with Frobenius by Corollary 4.17 .

REMARK. The isomorphism (5.5.1) should hold without assuming that $f_{0}^{-1}(W)=Z$ if we replace $f_{+}$by $f_{\text {! }}$. For this, we need to assume the Berthelot conjecture. In the following, we freely use this conjecture. Let us sketch a proof. It suffices to show the case where $f:(\mathscr{C}, Z) \rightarrow\left(\mathscr{C}, Z^{\prime}\right)$ such that the realization is the identity and $Z^{\prime} \subset Z$. We can see easily that it suffices to show that for $\mathscr{\mathscr { C }} \in \mathcal{C}_{\left(\mathscr{X}, \mathcal{Z}^{\prime}\right)}$ and $\mathscr{N} \in \mathcal{C}_{(\mathscr{C}, \mathcal{Z})}$, the homomorphism induced by scalar extension

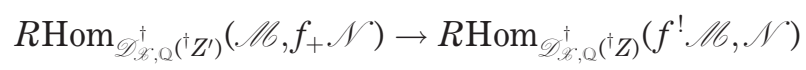

is an isomorphism. We can reduce the verification to the following two cases; when the support of $\mathscr{C} 6$ is contained in $Z$, and when $\mathscr{L}$ is a $\mathscr{D}_{\mathscr{L}, Q}^{\dagger}\left({ }^{\dagger} Z\right)$ module. To see the former case, use the theorem of Berthelot-Kashiwara [BeI, 5.3.3]. To see the latter case, it suffices to show that the homomorphism

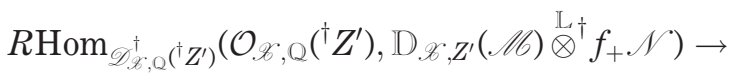

$$
\begin{aligned}
& \left.R \mathrm{Hom}_{\mathscr{Q} \mathscr{Q}, Q}^{\dagger}{ }^{\dagger} Z\right)\left(\mathcal{O}_{\mathscr{X}, \mathrm{Q}}\left({ }^{\dagger} Z\right), \mathrm{D}_{\mathscr{K}, Z}(\mathscr{C}) \stackrel{\mathrm{L}^{\dagger} \dagger}{\otimes} \mathscr{N}\right)
\end{aligned}
$$

is an isomorphism. Using the Spencer resolution, it suffices to show that the

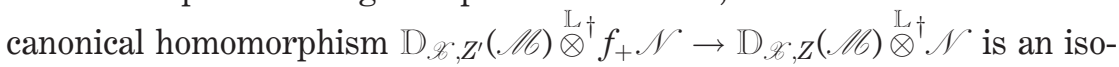
morphism. The verification is easy. 
Explicit calculation of Frobenius isomorphisms and Poincaré duality etc. 145

To complete the Poincaré duality we need to calculate $f^{!}$in the case where $f$ is smooth. Namely, we get the following.

THEOREM. Let $f:(\mathscr{B}, Z) \rightarrow(\mathscr{H}, W)$ be a morphism of d-couples such that the realization is smooth. Then there is a canonical isomorphism of cohomological functors with Frobenius isomorphisms

$$
f^{!} \cong f^{+}(d)[2 d]: D_{\mathrm{coh}}^{b}\left(\mathscr{D}_{\mathscr{H}, \mathrm{Q}}^{\dagger}\left({ }^{\dagger} W\right)\right) \rightarrow D_{\mathrm{coh}}^{b}\left(\mathscr{D}_{\mathscr{K}, \mathrm{Q}}^{\dagger}\left({ }^{\dagger} Z\right)\right)
$$

where d denotes the relative dimension of $f$.

Proof. Put the functor $D_{\mathscr{C}, Z}$ to the both sides of the isomorphism of Theorem 3.10. Using the involutivity [Vi, II.3.5] of $\mathrm{D}_{\mathscr{C}, Z}$, we get the claim.

5.6. By using the comparison between dual functor of arithmetic $\mathscr{D}$-modules and that of isocrystals, we can prove a purity type result. Namely we have

Theorem (Purity). Let $(\mathscr{C}, Z) \rightarrow(\mathscr{Y}, W)$ be a morphism of d-couple. Moreover, suppose that the realization off is a closed immersion. Let $\mathscr{l}$ be a convergent $F$-isocrystal on $\mathscr{H}$ overconvergent along $W$. Then $f^{+}(\mathscr{C})$ is defined, and we get

$$
f^{!}(\mathscr{C b}) \cong f^{+}(\mathscr{C O})(-d)[-2 d]
$$

where d denotes the codimension of $\mathscr{C}$ in $\mathscr{Y}$.

Proof. We know that $f^{*}\left(\operatorname{sp}^{*}(\mathscr{C})^{\vee}\right) \cong\left(f^{*} \operatorname{sp}^{*}(\mathscr{C})\right)^{\vee}$. Together with the comparison theorem of duality functors Corollary 3.12 and the compatibility of pull-backs [Ca5, 4.1.8], the theorem follows.

5.7. Consider the following cartesian diagram of d-couples.

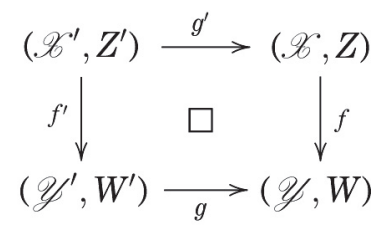

Here, cartesian means it is cartesian as a diagram of underlying formal schemes and $\mathscr{C}^{\prime} \backslash Z^{\prime} \cong(\mathscr{C} \backslash Z) \times(\mathscr{H} \backslash W)\left(\mathscr{H}^{\prime} \backslash W^{\prime}\right)$. Now, we get the following base change theorem. 
Theorem (Base change). We preserve the notation. Assume $g$ is a smooth morphism. Then we get a canonical equivalence of functors

$$
g^{!} \circ f_{+} \cong f_{+}^{\prime} \circ g^{\prime !}: \underline{L D}_{\mathrm{Q}, \mathrm{qc}}^{b}\left(\widehat{\mathscr{D}}_{\mathscr{\mathscr { C }}}^{(\bullet)}(Z)\right) \rightarrow \underline{L D_{\mathrm{Q}, \mathrm{qc}}^{b}}\left(\widehat{\mathscr{D}}_{\mathscr{Y}^{\prime}}^{(\bullet)}\left(W^{\prime}\right)\right) .
$$

This isomorphism is compatible with Frobenius pull-backs.

Proof. This follows by Lemma 2.6.

REMARK. (i) Let us assume Berthelot's conjecture. The theorem holds

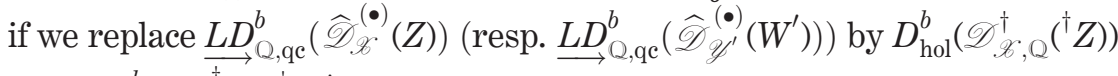
(resp. $\left.D_{\text {hol }}^{b}\left(\mathscr{D}_{\mathscr{Y}^{\prime}, Q}^{\dagger}\left({ }^{\dagger} W^{\prime}\right)\right)\right)$. When $g$ is a closed immersion, the theorem follows by Remark 2.6. Using the standard factorization of $g$ into a closed immersion followed by a smooth morphism, we define the isomorphism in the general case by composition. We need to show that the definition does not depend on the choice of factorizations. For this, use the commutativity of (2.6.4). Since the argument is standard, we leave the details to the reader.

(ii) Let $\mathscr{C} 6$ be an object in $D_{\text {coh }}^{b}\left(\mathscr{D}_{\mathscr{Q}, Q}^{\dagger}\left({ }^{\dagger} Z\right)\right)$. When $g^{+} \circ f_{!}(\mathscr{C l})$ and $f_{!}^{\prime} \circ g^{\prime+}(\mathscr{C l})$ are defined, the above equivalence and the isomorphism $\mathrm{D} \circ \mathrm{D} \cong \mathrm{id}$ induces an isomorphism

$$
g^{+} \circ f_{!}(\mathscr{L C}) \cong f_{!}^{\prime} \circ g^{\prime+}(\mathscr{C}) .
$$

5.8. We preserve the notation. Let $\mathscr{C} 6$ and $\mathscr{N}$ be perfect $(F-)_{\mathscr{Y}, Q}^{\dagger}\left({ }^{\dagger} W\right)-$ complexes. We assume that $\mathrm{D}_{\mathscr{Y}, W}(\mathscr{C O}) \stackrel{\mathrm{L}^{\dagger}}{\otimes} \mathrm{D}_{\mathscr{Y}, W}(\mathscr{N})$ is also perfect $(F-) \mathscr{D}_{\mathscr{Y}, Q}^{\dagger}\left({ }^{\dagger} W\right)$-complex. Then, we define the twisted tensor product of $\mathscr{L}$ and $\mathscr{N}$ denoted by $\mathscr{L} \widetilde{\otimes}^{\mathrm{L} \dagger} \mathscr{N}$ to be

$$
\mathrm{D}_{\mathscr{Y}, W}\left(\mathrm{D}_{\mathscr{Y}, W}(\mathscr{C O}) \stackrel{\mathrm{L} \dagger}{\otimes} \mathrm{D}_{\mathscr{Y}, W}(\mathscr{N})\right) .
$$

One of the reasons we introduce this twisted tensor product is the following.

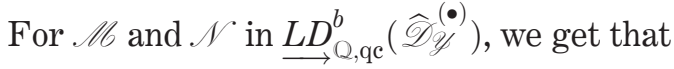

$$
f^{!}\left(\mathscr{L} \mathscr{L}^{\mathrm{L} \dagger} \mathscr{N}\right)\left[d_{f}\right] \cong f^{!} \mathscr{\mathscr { L }} \stackrel{\mathrm{L}}{\otimes} \dagger^{\dagger} f^{!} \mathscr{N}
$$

where $d_{f}:=\operatorname{dim}(\mathscr{C})-\operatorname{dim}(\mathscr{Y})$. This is compatible with the Frobenius structures. However, if we replace $f^{!}$by $f^{+}$, the equality (5.8.1) does not hold in general. Nevertheless, if we also replace $\stackrel{\mathrm{L}}{\otimes}^{\dagger}$ by $\widetilde{\otimes}^{\mathrm{L} \dagger}$, the equality holds in turn. Namely, (5.8.1) induces an isomorphism

$$
f^{+}\left(\mathscr{L} \widetilde{\otimes}^{\mathrm{L}_{\dagger}} \mathscr{N}\right)\left[-d_{f}\right] \cong f^{+} \mathscr{M} \widetilde{\otimes}^{\mathrm{L}_{\dagger}} f^{+} \mathscr{N}
$$

if the both sides are defined. A consequence of Lemma 4.3 is the following. 
Explicit calculation of Frobenius isomorphisms and Poincaré duality etc. 147

Proposition. Let $(\mathscr{B}, Z)$ be a d-couple, and $\mathscr{l}$ and $\mathscr{N}$ be coherent $F$ $\mathscr{D}_{\mathscr{X}, Q}^{\dagger}\left({ }^{\dagger} Z\right)$-complexes. Assume further that $\mathscr{L}$ is an overconvergent isocrystal along the divisor $Z$. Then we get

$$
\mathscr{L} \widetilde{\otimes}^{\mathrm{L} \dagger} \cdot \mathscr{N} \cong\left(\mathscr{L} \stackrel{\mathrm{L}}{\mathrm{L}} \dagger^{\dagger} \mathscr{N}\right)(d)
$$

where d denotes the dimension of $\mathscr{6}$.

5.9. Finally, let us compare the rigid cohomology with the push-forward as arithmetic $\mathscr{D}$-modules.

Let $\mathscr{C}$ be a proper smooth formal scheme of dimension $d, Z$ be a divisor of the special fiber of $\mathscr{C}, \mathscr{C}$ be the complement, and $U_{0}$ be its special fiber. We denote by $f:(\mathscr{C}, Z) \rightarrow(\operatorname{Spf}(R), \emptyset)$ the morphism of d-couples induced by the structural morphism of $\mathscr{C}$. Let $\mathscr{C}$ be a coherent $\mathscr{D}_{\mathscr{X}, \mathrm{Q}}^{\dagger}\left({ }^{\dagger} Z\right)$-module which is an overconvergent isocrystal along $Z$. Suppose that it is coherent as a $\mathscr{D}_{\mathscr{C}, Q}^{\dagger}$-module.

By Corollary 3.14, we get the canonical isomorphism

$$
H^{i} f_{+} \mathscr{C l} \cong H_{\text {rig }}^{d+i}\left(U_{0}, \operatorname{sp}^{*}(\mathscr{C l})\right)(d) \text {. }
$$

To see the relation for cohomologies with compact supports, we use the Poincaré duality of rigid cohomology. In the curve case, Poincaré duality is proven in [Cr]. In the general case, we could not find any literature explicitly stating the Poincaré duality with Frobenius structure. However in [St, 8.3.14], the coupling is defined, and in [Ke], the perfectness of the couple is proven. Thus we get the following isomorphism

$$
H_{\text {rig }}^{i}\left(U_{0}, M\right)^{\vee} \cong H_{\text {rig }, c}^{2 d-i}\left(U_{0}, M^{\vee}(d)\right)
$$

for an overconvergent $F$-isocrystal $M$ on the smooth variety $U_{0}$ over $k$. Using this, we get

$$
\begin{aligned}
& H^{i} f_{!} \mathscr{C l} \cong\left(H^{-i} f_{+} \mathrm{D}_{\mathscr{C}, Z}(\mathscr{C l})\right)^{\vee} \cong \\
&\left(H_{\text {rig }}^{d-i}\left(U_{0}, \operatorname{sp}^{*}(\mathscr{C l})^{\vee}(-d)\right)(d)\right)^{\vee} \cong H_{\text {rig, }, c}^{d+i}\left(U_{0}, \operatorname{sp}^{*}(\mathscr{C})\right)(d) .
\end{aligned}
$$

Here the second isomorphism follows from Corollary 3.12. Summing up, we get

$$
H^{i} f_{+} \mathscr{C l} \cong H_{\text {rig }}^{d+i}\left(U_{0}, \operatorname{sp}^{*}(\mathscr{C l})\right)(d), \quad H^{i} f_{!} \mathscr{C l} \cong H_{\text {rig }, c}^{d+i}\left(U_{0}, \operatorname{sp}^{*}(\mathscr{C l})\right)(d) .
$$

In particular, we note that there exist canonical isomorphisms

$$
H^{i} f_{+} f^{+}(K) \cong H_{\text {rig }}^{i}\left(U_{0} / K\right), \quad H^{i} f_{1} f^{+}(K) \cong H_{\text {rig }, c}^{i}\left(U_{0} / K\right)
$$

compatible with Frobenius isomorphism. 
Acknowledgments. The author would like to thank Professor P. Berthelot for letting him know the proof of the Künneth formula. Most of the work of this paper was done when the author was visiting to IRMA of Université de Strasbourg in 2010. He would like to thank A. Marmora and the institute for the hospitality. He also like to express his gratitude to Professor A. Shiho for stimulating discussions. This work was supported by Grant-in-Aid for JSPS Fellows 20-1070, and partially by l'agence nationale de la recherche ANR-09-JCJC-0048-01.

\section{REFERENCES}

[Ab1] ABE T., Coherence of certain overconvergent isocrystals without Frobenius structures on curves, Math. Ann. 350, pp. 577-609 (2011).

[Ab2] ABE T., Langlands correspondence for isocrystals and existence of crystalline companion for curves, available at arxiv.org/abs/1310.0528.

[AC] ABE T. - CARo D., Theory of weights in p-adic cohomology, available at arxiv.org/abs/1303.0662.

[AM] ABE T. - MaRMORA A., On p-adic product formula for epsilon factors, To appear in J. Inst. Math. Jussieu.

[BB] Baldassarri F. - Berthelot P., On Dwork cohomology for singular hypersurfaces, Geometric aspects of Dwork theory, vol. I, pp. 177-244, Walter de Gruyter (2004).

[BeP] Berthelot P., Cohomologie rigide et cohomologie rigide à supports propres. Première partie, preprint.

[Be1] Berthelot P., D-modules arithmétiques I. Opérateurs différentiels de niveau fini, Ann. Sci. École Norm. Sup. 4ème série, 29 no. 2, pp. 185-272 (1996).

[Be2] Berthelot P., D-modules arithmétiques II. Descente par Frobenius, Mém. Soc. Math. Fr. 81 (2000).

[BeI] Berthelot P., Introduction à la théorie arithmétique des $\mathscr{D}$-modules, Asterisque 279, pp. 1-80 (2002).

[Ca1] Caro D., D-modules arithmétiques surcohérents. Application aux fonctions L, Ann. Inst. Fourier 54, pp. 1943-1996 (2005).

[Ca2] Caro D., Fonctions L associées aux D-modulles arithmétiques. Cas des courbes, Compos. Math. 142, pp. 169-206 (2006).

[Ca3] CARo D., Comparaison des foncteurs duaux des isocristaux surconvergents, Rend. Sem. Mat. Univ. Padova 114, pp. 131-211 (2006).

[Ca4] Caro D., Dévissages des F-complexes de $\mathscr{D}$-modules arithmétiques en F-isocristaux surconvergents, Invent. Math. 166 no. 2, pp. 397-456 (2006).

[Ca5] CARo D., D-modules arithmétiques associés aux isocristaux survonvergents. Cas Lisse, Bull. Soc. Math. Fr. 137 no. 4, pp. 453-543 (2009).

[Ca6] CARo D., D-modules arithmétiques surholonomes, Ann. Sci. École Norm. Sup. 4ème série, 42 no. 1, pp. 141-192 (2009).

[Ca7] Caro D., Sur la compatibilité à Frobenius de l'isomorphisme de dualité relative, Rend. Sem. Mat. Univ. Padova 122, pp. 235-286 (2009). 
Explicit calculation of Frobenius isomorphisms and Poincaré duality etc. 149

[Co] Conrad, B., Grothendieck Duality and Base Change, Lecture Notes in Math. 1750, Springer (2000).

[Cr] CREw R., Finiteness theorems for the cohomology of an overconvergent isocrystal on a curve, Ann. Sci. École Norm. Sup. 4ème série, 31 no. 6, pp. 717-763 (1998).

[Ga] Garnier L., Descente par Frobenius explicite pour les $\mathscr{D}^{\dagger}$-modules, J. Algebra 205, pp. 542-577 (1998).

[GK] Gross B. - Koblitz N., Gauss sums and the p-adic $\Gamma$-function, Ann. Math. 109, pp. 569-581 (1979).

[Ha] Hartshorne R., Residues and Duality, Lecture Notes in Math. 20, Springer (1966).

[NH1] Noot-Huyghe C., Transformation de Fourier des $\mathcal{D}$-modules arithmétiques I, Geometric aspects of Dwork theory. vol. II, pp. 857-907, Walter de Gruyter (2004).

[NH2] Noot-Huyghe C., Finitude de la dimension homologique d'algèbres d'opérateurs différentiels faiblement complètes et à coefficients surconvergents, J. Algebra 307, pp. 499-540 (2007).

[Ka] Kashiwara M., Senkei-henbibun-houteishiki-kei no daisuuteki kenkyuu, Master thesis, Univ. of Tokyo (1970).

[Ke] Kedlaya K.S., Finiteness of rigid cohomology with coefficients, Duke Math. J. 134, pp. 15-97 (2006).

[Kl] Kleiman S.L., Relative duality for quasi-coherent sheaves, Compos. Math. 41, pp. 39-60 (1980).

[St] Le Stum B., Rigid cohomology, Cambridge Tracts in Math. 172, Cambridge University Press (2007).

[Vi] VIRRION A., Dualité locale et holonomie pour les $\mathscr{D}$-modules arithmétiques, Bull. Soc. Math. Fr. 128, pp. 1-68 (2000).

[Vi2] VIRRIOn A., Trace et dualité relative pour les $\mathscr{D}$-modules arithmétiques, Geometric aspects of Dwork theory. vol. II, pp. 1039-1112, Walter de Gruyter (2004).

Manoscritto pervenuto in redazione il 3 Settembre 2012. 
